\title{
Cluster Growth During De-Wetting of Solid Thin Films: Coarsening Exponents and the Role of Long Range De-Wetting Interactions
}

Adi Constantinescu

West Virginia University

Follow this and additional works at: https://researchrepository.wvu.edu/etd

\section{Recommended Citation}

Constantinescu, Adi, "Cluster Growth During De-Wetting of Solid Thin Films: Coarsening Exponents and the Role of Long Range De-Wetting Interactions" (2011). Graduate Theses, Dissertations, and Problem Reports. 3460.

https://researchrepository.wvu.edu/etd/3460

This Dissertation is protected by copyright and/or related rights. It has been brought to you by the The Research Repository @ WVU with permission from the rights-holder(s). You are free to use this Dissertation in any way that is permitted by the copyright and related rights legislation that applies to your use. For other uses you must obtain permission from the rights-holder(s) directly, unless additional rights are indicated by a Creative Commons license in the record and/ or on the work itself. This Dissertation has been accepted for inclusion in WVU Graduate Theses, Dissertations, and Problem Reports collection by an authorized administrator of The Research Repository @ WVU.

For more information, please contact researchrepository@mail.wvu.edu. 


\title{
Cluster Growth During De-Wetting of Solid Thin Films: Coarsening Exponents and the Role of Long Range De-Wetting Interactions
}

\author{
Adi Constantinescu \\ Dissertation submitted to the \\ Eberly College of Arts and Sciences \\ at West Virginia University \\ in partial fulfillment of the requirements \\ for the degree of
}

Doctor of Philosophy

in

Physics

Leonardo Golubovic, Ph.D., Chair

Sergei Urazhdin, Ph.D.

Wathiq Abdul-Razzaq, Ph.D.

Harry Gingold, Ph.D.

Feruz Ganikhanov, Ph.D.

Department of Physics

\section{Morgantown, West Virginia \\ 2011}

Keywords: Casimir Interactions, Long-range Interactions, Dewetting Phenomena,

Thin Films, Non-equilibrium Statisitical Physics, Coarsening Processes, Cluster Growth

Copyright 2011 Adi Constantinescu 


\section{ABSTRACT \\ Cluster growth during de-wetting of solid thin films: Coarsening exponents and the role of long range de-wetting interactions}

\section{Adi Constantinescu}

Coarsening processes play prominent roles in non-equilibrium statistical physics and in applied physical sciences. A new area in this field of statistical physics has emerged from recent experimental revelations that long range de-wetting forces acting across thin films, such as the fundamental van der Waals interactions, may drive the formation of large clusters (tall multilayer islands) and pits, observed in thin films of soft materials (polymers), as well as in thin films of liquid and solid metals. Motivated by the experiments, in this Thesis we elucidate the fundamentals of the non-equilibrium statistical mechanics of solid thin films coarsening within a unified model explicitly incorporating de-wetting interactions. By analytic arguments and simulations of the model, we study the growth laws of clusters formed in thin films due to the de-wetting interactions. The ultimate long time-scale cluster growth coarsening exponents are found to depend on the substrate dimensionality, unlike the super-universal exponents encountered in standard coarsening phenomena. Nonetheless, the ultimate cluster growth scaling laws at long times are strongly universal: Short and long range de-wetting interactions yield the same coarsening exponents. However, long range de-wetting interactions, such as the common van der Waals forces, introduce a distinct long lasting early-time scaling behavior characterized by a slow growth of the cluster height/lateral size aspect ratio (i.e., a time-dependent Young angle), and by effective coarsening exponents that depend on cluster size. In this thesis, we develop a theory capable to calculate these effective size-dependent coarsening exponents characterizing the cluster growth in the early-time cross-over regime. Such a pronounced crossover behavior has been indeed seen in experiments; however its physical origin has remained elusive to this date. Our results attribute these observed phenomena to ubiquitous long range dewetting interactions acting across thin films. 


\section{Curriculum Vitae}

\section{ADI CONSTANTINESCU}

\section{EDUCATION}

Ph.D. Physics, West Virginia University, Spring 2011 (expected)

M.S. Physics, University of Bucharest, Romania, 2001

B.S. Physics, University of Bucharest, Romania, 1998

\section{PROFESSIONAL EXPERIENCE}

2003-present Teaching Assistant and Ph.D. Candidate, West Virginia University Theoretical Condensed Matter Physics: Cluster growth during de-wetting of solid thin films: Coarsening exponents and the role of long range de-wetting interactions.

2001-2003 Research Assistant, University of Missouri-Columbia Theoretical Condensed Matter Physics: Time dependent spin density functional theory,

1999-2001 M.S. student ; M.S. Thesis: On the specific heat of type I binary metallic alloys-A: simulation study, University of Bucharest, Romania.

1993-1998 B.S. student; B.S. Thesis: The Coulomb Green's function and multi-phonon Calculations, University of Bucharest, Romania.

\section{Adi Constantinescu: MY RESEARCH INTERESTS AND ACHIEVEMENTS}

Statistical Mechanics and Condensed Matter Theory: Modern theories of phase transitions, Non-Equilibrium Statistical Mechanics; Phase ordering phenomena; Dynamics of surfaces and interfaces; Theory of many-particle systems in external fields, confined geometry and reduced dimensionality.

Dynamics of Physical Systems: Analytic, Computational, and Numerical Methods: Algorithm development; Large scale numerical simulations; Grid methods for continuum modeling; Molecular Dynamics and Monte Carlo simulations. 
My research interest is the behavior of complex systems at the interface between physics and the fields of biology and materials science. My recent work is dedicated to elucidating the coarsening dynamics of thin films of solids (such as soft polymeric and metallic materials) within a unified model that incorporates long range de-wetting interactions acting across thin films (such as the ubiquitous van der Walls forces). By analytic arguments and simulations, I study the growth laws of clusters formed in these films due to the de-wetting interactions. I have revealed that long range de-wetting interactions introduce a long lasting early time scaling behavior characterized by a slow growth of the cluster height/lateral size aspect ratio. Such an interesting cluster growth behavior (characterized by a time dependent Young angle) has been indeed seen in experiments, however its physical origin has remained unexplained to this date. My theoretical study explains this phenomenon as an effect of the long range de-wetting interactions acting across thin films. I have described my results in this area in my Publication 1 and in my presentations 2 through 6.

I have been interested also in correlated quantum systems. I this area, I studied the dynamical effects that arise from the interaction between electrons, by using dynamical density functional theory. I contributed to the development of the formalism known as "current-density functional theory", which expresses the transport properties of electron systems in terms of their current density. I have described my results in this area in my Physical Review Letters paper (Publication 2).

The study of specific heats of alloys, by theory and simulation, was another area of interest to me. In my M.S. thesis, I employed the lattice dynamical and Monte Carlo approaches to study the variation of the specific heat of Type I binary metallic alloys with changing the concentration of the minority phase. My study revealed significant deviations from linearity of the low-temperature specific heat, which cannot be explained by the conventional Debye's low temperature contribution. Rather, they were shown to emerge due to anharmonic phonon vibrations.

\section{PUBLICATIONS}

1. Adi Constantinescu, Leonardo Golubović, and Artem Levandovsky, "Cluster growth during de-wetting of solid thin films.: Coarsening exponents and the role of long range de-wetting interactions" (submitted journal article)

2. Zhixin Qian, Adi Constantinescu and Giovanni Vignale

"Solving the ultra-nonlocality problem in time-dependent spin-density functional theory", Physical Review Letters 90, 066402 (2003) 


\section{SEMINARS, COLLOQUIA, AND TALKS}

1. Zhixin Qian, Adi Constantinescu, Giovanni Vignale,

"Solving the ultra-nonlocality problem in time-dependent spin-density functional theory", at the March Meeting of the American Physical Society, Austin, Texas, March 2003. (oral presentation)

2. Adi Constantinescu, Artem Levandovsky, Leonardo Golubović, "Morphology evolution of solid thin films in the presence of long range de-wetting interactions", in the special Focus Session U20: Metal Surfaces, Interfaces, and Thin Films, of the March Meeting of the American Physical Society, New Orleans, March 2008 (oral presentation)

3. Adi Constantinescu, Leonardo Golubović, and Artem Levandovsky, "Interface evolution and cluster formation during de-wetting of thin solid films", at the March Meeting of the American Physical Society, Pittsburgh, Pennsylvania, March 2009. (oral presentation)

4. Adi Constantinescu, Leonardo Golubović, and Artem Levandovsky, "Cluster growth driven by long-range de-wetting interactions acting across thin films" at Complex Driven Systems - From Statistical Physics to Life Sciences, Virginia Tech international conference organized in honor of Professor Royce Zia , Virginia Tech, October 2010. (oral presentation)

5. Adi Constantinescu, Leonardo Golubović, and Artem Levandovsky, "Cluster growth driven by long-range de-wetting interactions in thin films" at the March Meeting of the American Physical Society, Dallas, Texas, March $2010 . \quad$ (poster presentation)

\section{Leonardo Golubović and Adi Constantinescu,}

"Spinodal de-wetting of thin films in the presence of oscillatory Casimir forces" in the special Focus Session A31: van der Waals Bonding in Advanced Materials: Fundamentals and Simple Systems, of the March Meeting of the American Physical Society, Dallas, Texas, March 2010 (oral presentation) 


\section{Acknowledgements}

I would like to express my deep gratitude to my advisor Dr. Leonardo Golubovic for providing me with the opportunity to do my research in a fascinating physics area. I am grateful to him for his guidance, patience, inspirations, and productive discussions. It is certain I learnt more than just theoretical physics from him. I am also grateful to Dr. Jorge Espinosa and Dr. David Lederman, for the inspirations I gained by reading their experimental studies, and to Dr. Artem Levandovsky, for the numerous discussions we had on the topics discussed in this thesis.

I would like to thank Dr. Sergei Urazhdin, Dr. Wathiq Abdul-Razzaq, Dr. Feruz Ganikhanov, and Dr. Harry Gingold for taking time from their busy schedules to serve on my committee. I would like to express my special gratitude to the former member of my committee, late Dr. Andrzej Karwowsky whose spirit of scientific integrity and free mind will be missed forever.

Finally, thanks to my great parents and my dear wife, for all the courage and happiness they have provided me. 


\section{Contents}

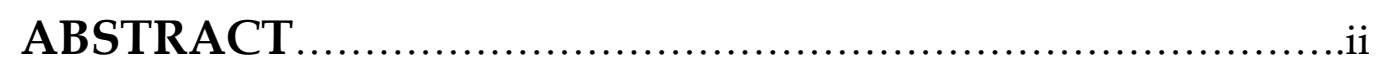

Curriculum Vitae.....................................................ii

Acknowledgement.............................................. vi

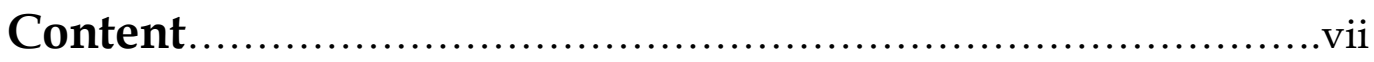

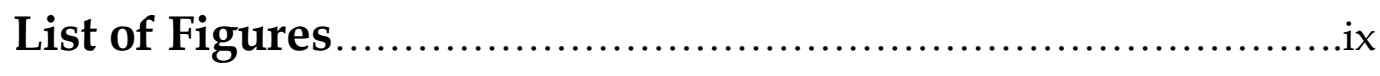

Chapter 1 Introduction

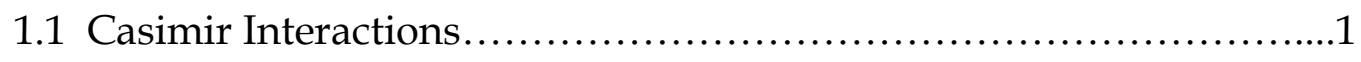

1.2 Coarsening of Thin Films and this Thesis goals...................... 3

1.3 References...................................................6

Chapter 2 Interface Dynamics Model with De-wetting Interactions

2.1 The Model.....................................................

2.2. Spinodal Instability ..........................................

2.3 Young Contact Angle..........................................13

2.4 Rescaled Model................................................18

2.5 Cluster Coarsening Dynamics in the Young-Laplace Limit...........23

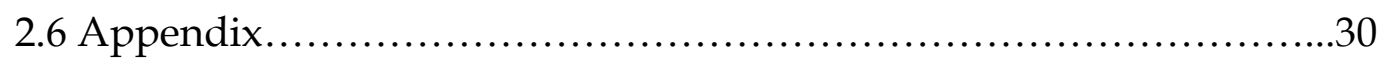

2.7. References....................................................36 


\section{Chapter 3 Simulations of interface dynamics and the cluster coarsening growth}

3.1 Introduction.

3.2 Statistical quantities characterizing clusters and thin film interfaces....41

3.3 Dynamics of cluster sizes and pressure

3.4 Other statistical properties of the thin film interface 53

Chapter 4: Clusters morphology beyond the classical Young-Laplace model

4.1 Introduction .63

4.2 Quasi-static clusters..............................................65

4.3 Appendix........................................................ 74

\section{Chapter 5 Analytic Theory of the Cluster Coarsening Growth}

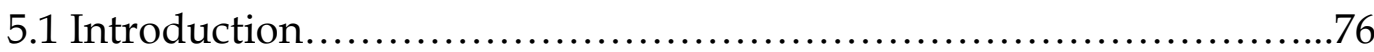

5.2 Cluster dynamics and statistics.................................77

5.3 Cluster distribution cut off.......................................... 79

5. 4 Closing the cut off dynamics equation............................ 81

5.5 Calculating effective coarsening exponents........................ 83

5.6 Young-Laplace model revisited $\ldots \ldots \ldots \ldots \ldots \ldots \ldots \ldots \ldots \ldots \ldots \ldots \ldots . \ldots \ldots$

5.7 Effective exponents beyond the Young-Laplace model...............90

5.8 References....................................................... 100

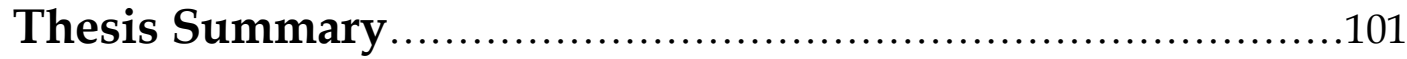




\section{List of Figures}

\section{Chapter 2:}

Fig. 1. De-wetting interaction potential causing the flat film instability.

Fig. 2. Schematic view of small fluctuations $\delta h$ of the thin film surface around average thickness $h_{0}$.

Fig. 3. Variation of the dispersion relation $G(q)$ as function of the magnitude of the wavevector $q$.

Fig. 4. A snapshot from our simulations: The interface profile $\tilde{h}(x, t)=h(x, t)-h_{\min }$ (thinner line) in $D=1$ and corresponding field of pressure $P(x, t)$ (thicker line).

Fig. 5. Snapshots from our simulations: (a) The initial stage of the $D=2$ thin film interface, dominated by the formation of pits. Figure (b) exhibits the final stage of the $D=2$ thin film interface evolution, dominated by the presence of growing clusters.

Fig. 6. Sketch of a cluster on top of a substrate depicting the Young-Dupré model of dewetting; $\gamma, \gamma_{F S}$ and $\gamma_{V S}$ are the interface energies at vacuum-film, film-substrate and vacuum-substrate interfaces, respectively ; $\theta_{Y}$ is the cluster-substrate contact angle and $R_{1}$ is the radius of the curvature of the cluster interface with vacuum whereas $R$ is the radius of the cluster circular base.

Fig. 7. Interface height plots showing the formation of a slowly growing cluster for $D=1$ and $\omega=1$, from our simulation of the rescaled model with dimensionless $t, \vec{x}$ and $h$. Here, $T$ is a characteristic time-scale at which the well structured clusters are seen for the first time. In the figure, we indicate tangents drawn at inflection points of the cluster profiles. Note that the tangent slope slowly grows in time. 
Fig. 8. Interface height plots showing the formation of a slowly growing cluster for $D=2$ and $\omega=1$, from our simulation of the rescaled model with dimensionless $t, \vec{x}$ and $h$ [as in Fig. 5(b)]. Here, $T$ is a characteristic time-scale at which the well structured clusters are seen for the first time. It corresponds to the crossover from pits arrays [Fig. 5(a)] to cluster dominated surface morphology [Fig. 5(b)]. In the figure, we indicate tangents drawn at inflection points of the cluster profiles. Note that the tangent slope slowly grows in time.

Fig. 9. (a) Schematic representation of a configuration with no atom added on the surface. The free energy of this configuration is labeled as $F_{\text {without }}$. In (b) we represent the configuration with the atom added on the surface at position $\vec{x}_{0}$, causing the interface height change $\delta h$. The free energy of this configuration is labeled as $F_{\text {with }}$. The difference of the free energy between these two configurations is the chemical potential field: $\mu\left(x_{0}\right)=F_{\text {with }}-F_{\text {without }}$. In figure (c) we represent schematically the surface atoms and $x$-components of their velocities at positions $x$ and $x+\Delta x$, respectively. Figure (d) shows the tangential force causing drift of surface atoms.

\section{Chapter 3:}

Fig. 1. Figure (a) exhibits the final stage of the $D=2$ thin film interface evolution, dominated by the presence of growing clusters. In figure (b), interface height contour plots showing film morphology evolution for $D=2$ and $\omega=1$, from the simulation of the rescaled model with dimensionless $t, \vec{x}, h$ (see Sec. 2.4). Here, $T$ is a time-scale conventionally set to correspond to the emergence of the transient worm-like morphology at $t \approx 3 T$. For $t<3 T$, the interface is in a time stage characterized by pit formation [as in Fig. 5(a) of Ch. 2]. For $t>3 T$, the interface is characterized by the formation of slowly growing clusters [as in figure (a) here].

Fig. 2. Time evolution of the number of clusters $N(t)$ in the systems we simulated, with $\omega=1,2$, and 6 . The $N(t)$ displayed here is the average of the clusters numbers obtained from ten simulations done for each value of $\omega$. Also presented is the effective coarsening exponent, $\beta_{N}(t)=d \ln [N(t)] / d \ln (t)$. This exponent approaches the value of $-3 / 4$ at long 
times. There are however significant deviations away from this value, that are seen in the $\beta_{\mathrm{N}}(t)$ at early times.

Fig. 3. Temporal evolution of the clusters average volume $\langle V\rangle$ in the systems we simulated, with $\omega=1,2$, and 6 . We also present the evolution of the corresponding effective coarsening exponent, $\beta_{<V>}(t)=d \ln [<V>] / d \ln (t)$. This exponent approaches the value of $+3 / 4$ at long times. There are however significant deviations away from this value, that are seen in the $\beta_{<\mathrm{V}>}(\mathrm{t})$ at early times.

Fig. 4. Temporal evolution of the clusters average pressure $\langle P>$ in the systems we simulated, with $\omega=1,2$, and 6 . We also present the evolution of the corresponding effective coarsening exponent, $\beta_{<P>}(t)=d \ln [<P>] / d \ln (t)$. This exponent approaches the value of $-1 / 4$ at long times. There are however significant deviations away from this value, that are seen in the $\beta_{<P>}(t)$ at early times.

Fig. 5. Time evolution of the clusters average height $\langle H\rangle$ in the systems we simulated, with $\omega=1,2$, and 6 . We also present the evolution of the corresponding effective exponent, $\beta_{<\mathrm{H}>}(\mathrm{t})=\mathrm{d} \ln [<\mathrm{H}>] / \mathrm{d} \ln (\mathrm{t})$. This exponent approaches the value of $+1 / 4$ at long times. There are however significant deviations away from this value, that are seen in the $\beta_{<\mathrm{H}>}(\mathrm{t})$ at early times.

Fig. 6. Time evolution of the clusters average base radius $<R>$ in the systems we simulated, with $\omega=1,2$, and 6 . We also present the evolution of the corresponding effective exponent, $\beta_{R}(t)=d \ln [<R>] / d \ln (t)$. This exponent approaches the value of $+1 / 4$ at long times. There are however significant deviations away from this value, that are seen in the $\beta_{<R>}(t)$ at early times. For comparison, we also display $\beta_{<A>}(t) / 2$, where $\beta_{<A>}>(t)=d \ln [<A>] / d \ln (t)$ is the effective exponent for the growth of cluster base area.

Fig. 7: The figure qualitatively documents the correlation between the failure of the Young-Laplace model prediction for cluster aspect ratio (lower panel), with the magnitude of the deviations of the effective exponent $\beta_{H}$ away from its asymptotic value (upper panel). Both effects increase with decreasing $\omega$. 
Fig. 8. The time evolution of the area fraction occupied by clusters, i.e., the wet area fraction $\phi$ in the systems we simulated, with $\omega=1,2$, and 6 . In the figure, we also plot the corresponding effective coarsening exponent $\beta_{\phi}$. It approaches the value of $-1 / 4$ at long times.

Fig. 9. The time evolution of the restricted average height $h_{\text {rest }}$ in the systems we simulated, with $\omega=1,2$, and 6 . In the figure, we also plot the corresponding effective coarsening exponent. It approaches the value of $1 / 4$ at long times.

Fig. 10. Simulations results documenting that $-\beta_{N}(t)$ is approximately equal to $\beta_{<V>}(t)$.

Fig. 11. Simulations results documenting the Eq. (44)

Fig. 12. The height-height correlation function $K(r, t)$ obtained at several instants of evolution time. The most prominent feature of the $K(r, t)$ is its central peak originating from single cluster spatial self-correlations. The width $w(t)$ of the self-correlation peak is approximately equal to $2<R>$.

Fig. 13. Time evolution of the width $w(t)$ of the self-correlation peak of the correlation function $K(r, t)$ in the systems we simulated, with $\omega=1,2$, and 6 .

\section{Chapter 4:}

Fig. 1. (a) Cluster profiles obtained by solving the Eq. (3) for $\omega=1$, for several different pressures. To obtain the cluster type solutions, for each cluster height $H$, the pressure is fixed by the boundary condition that the interface height approaches a finite value at infinity. (b) The figure conceptualizes the definition of the cluster radius $R$. Also labeled is the inflection point of the cluster profile at which the interface slope reaches its maximum value $m_{\max }$. 
Fig. 2. Our numerical results for the function $P_{\text {stat }}(V)$ for the rescaled model with $\omega=1,2$, and 6 . The dashed line is the corresponding result in the Young-Laplace model; see Eqs. 65-67 of Ch. 2.

Fig. 3. Our numerical results for the function $H_{s t a t}(V)$ for the rescaled model with $\omega=1,2$, and 6 . The dashed line is the corresponding result in the Young-Laplace model; see Eqs. 65-67 of Ch. 2.

Fig. 4. Our numerical results for the function $R_{\text {stat }}(V)$ for the rescaled model with $\omega=1,2$, and 6 . The dashed line is the corresponding result in the Young-Laplace model; see Eqs. 65-67 of Ch. 2.

Fig. 5. The figure documents the way how the four cluster properties in Eqs. (9) through (12) approach the Young-Laplace limit with increasing cluster height $H$, for the rescaled model with $\omega=1,2$, and 6 .

\section{Chapter 5:}

Fig. 1. (a) The figure contains two plots. One of them is obtained from our simulations of the model with $\omega=1$, in $D=2$. It plots the obtained average cluster pressure $\langle\mathrm{P}>$ versus the average cluster volume $\langle\mathrm{V}\rangle$, obtained at various times. In the same figure, we give also the plot displaying the function $P_{\text {stat }}(V)$ as obtained in our analytic calculations in Chapter 4 , for $\omega=1$, in $D=2$ [see also Fig. 2 of Ch.4]. From the displays of the two plots, one can see that Eq. (21) holds for essentially all times [except for very early times, when the clusters are small and not quasi-static]. The figure (b) is the same as the figure (a) but for the model with $\omega=2$, in $D=2$

Fig. 2. Analytic results versus the corresponding simulation results for the effective coarsening exponent for the growth of cluster volume, $\beta<v>$ as function of the average cluster volume $\langle V\rangle$. We recall that the asymptotic value of $\beta<v>$ is 0.75 for infinite $\langle V\rangle$.

Fig. 3. Analytic results versus the corresponding simulation results for the effective coarsening exponent for the dynamics of cluster pressure, $\beta<p>$ as function of the 
average cluster volume $\langle V\rangle$. We note that the asymptotic value of $\beta<\mathrm{p}\rangle$ is -0.25 for infinite $<\mathrm{V}\rangle$.

Fig. 4. Analytic results versus the corresponding simulation results for the effective coarsening exponent for the growth of cluster height, $\beta_{<\mathrm{H}>}$ as function of the average cluster volume $\langle V\rangle$. We recall that the asymptotic value of $\beta_{<H}>$ is 0.25 for infinite $\langle V\rangle$.

Fig. 5. Analytic results versus the corresponding simulation results for the effective coarsening exponent for the growth of cluster base radius, $\beta_{<R>}$ as function of the average cluster volume $\langle V\rangle$. We recall that the asymptotic value of $\beta_{<R>}$ is 0.25 for infinite $<\mathrm{V}\rangle$.

Fig. 6. Analytic results for the effective coarsening exponents $\beta<V>, \beta<P>, \beta_{<H>\text {, and } \beta_{<R>} \text { as }}$ functions of the average cluster volume $\langle V\rangle$, for the rescaled model with $\omega=1$. In this figure, we display the exponents in a volume $\langle\mathrm{V}>$ range that is much wider than in figs. 2-5 so one can see the better the approach to the asymptotic exponents values, which are 0.75 for $\beta<\mathrm{V}>,-0.25$ for $\beta<P>, 0.25$ for $\beta<H>$, and 0.25 for $\beta_{<R>\text {. }}$

Fig. 7. Analytic results for the effective coarsening exponents $\beta<V>, \beta<P>, \beta<H>$, and $\beta_{<R>}$ as functions of the average cluster volume $\langle V\rangle$, for the rescaled model with $\omega=2$. In this figure, we display the exponents in a volume $<V>$ range that is much wider than in figs. 2-5 so one can see the better the approach to the asymptotic exponents values, which are 0.75 for $\beta_{<\mathrm{V}>},-0.25$ for $\beta_{<\mathrm{P}>}, 0.25$ for $\beta<H>$, and 0.25 for $\beta_{<\mathrm{R}>\text {. }}$

Fig. 8. Analytic results for the effective coarsening exponents $\beta<V>, \beta<P>, \beta<H>$, and $\beta_{<R>}$ as functions of the average cluster volume $\langle V\rangle$, for the rescaled model with $\omega=6$. In this figure, we display the exponents in a volume $\langle\mathrm{V}>$ range that is much wider than in figs. 2-5 so one can see the better the approach to the asymptotic exponents values, which are 0.75 for $\beta<\mathrm{V}>,-0.25$ for $\beta<P>, 0.25$ for $\beta<H>$, and 0.25 for $\beta_{<R>\text {. }}$ 


\section{Chapter 1}

\section{Introduction}

\subsection{Casimir Interactions}

In 1948, Hendrik Brugt Casimir using Quantum Electrodynamics made a striking prediction that two perfectly reflecting parallel plates placed in vacuum will attract each other [1]. This exotic phenomenon is due to the modification of the quantum electromagnetic field ground state ("zero point") energy between the plates which is produced simply by the presence of the plates. Indeed, the ground state quantum field energy of the empty space is different from that with plates present. Casimir calculated the difference between the two energies, with the result:

$$
\Phi(h)=-\frac{3 \pi^{2}}{240} \frac{\hbar c}{h^{3}},
$$

per unit area of the plates. This produces a net force per unit area (attractive pressure) of the form

$$
-\frac{\partial \Phi}{\partial h}=-\frac{\pi^{2}}{240} \frac{\hbar c}{h^{4}}
$$

where $\hbar$ is Planck's constant divided by $2 \pi, c$ is the speed of light and $h$ the separation between the two plates [1]. The origin of this force results from the fact that even in a perfect vacuum at zero absolute temperature there is a fluctuating electromagnetic field having a mean energy equal to half the energy of a photon (per mode). Consider the gap between the two plates as a cavity. Electromagnetic fields can 
propagate in space, they carry energy, and they also exert pressure on surfaces. This radiation pressure increases with the energy (and hence, the frequency) of the electromagnetic field. At a cavity-resonance frequency, the radiation pressure inside the cavity is stronger than outside and the plates are therefore pushed apart. Out of resonance, in contrast, the radiation pressure inside the cavity is smaller than outside and the plates are drawn towards each other. It turns out, on balance, that the attractive components have a slightly stronger impact than the repulsive ones.

Casimir's discovery had a great intellectual impact on the developments in the theoretical understanding of other forces in nature, in particular of the van der Waals forces. This deep understanding of the van der Waals forces was provided by Lifshitz who related these forces to electronic charge density fluctuations existing in any material even at zero absolute temperature due to quantum effects [2-4]. These charge fluctuations and the induced electromagnetic field fluctuations generate transient dipole moments in atoms and produce effective forces acting even between neutral atoms. At zero temperature these forces are called as London forces. The London force constitutes the major branch of Van der Waals interactions and for this reason it is called London-van der Waals force or bosonic Casimir interaction because they are all transmitted through photons. The London energy effective potential between two neutral atoms has the form

$$
\Phi(d)=-\frac{6 \pi \alpha^{2} \hbar v}{4\left(4 \pi \varepsilon_{0}\right)^{2} d^{6}}
$$

where $\alpha$ is the atomic polarizability, $v$ is a characteristic frequency (the optic absorption frequency), and $d$ is the distance between the two atoms. Hamaker considered this London-Van der Waals to discuss thin films [4]. He found that opposite surfaces of a thin film interact through the effective potential

$$
\Phi(h)=-\frac{A_{H}}{12 \pi h^{2}},
$$


per unit area, where $h$ is the thickness of the thin film, and $A_{H}$ is the so called Hamaker constant that can be calculated from the Lifshits theory $[2,3]$.

Recently, another interaction, the so called fermionic Casimir interaction was predicted to occur $[5,6]$. It is mediated by traveling electrons in metals, rather than by photons. In metallic thin films, this interaction is expected to be more important than the van der Walls interaction in Eq. (4), [7]. It has been proposed that the opposite surfaces of a metallic film interact with each other by an attractive fermionic Casimir interaction of the form:

$$
\Phi(h) \approx-\frac{\varepsilon_{F}}{a h},
$$

where $h$ is the thickness of the thin film, $\varepsilon_{F}$ is the Fermi energy of the metal, and $a$ is the atomic size.

\subsection{Coarsening of Thin Films and this Thesis goals}

Ever since a seminal study of Lifshits and Slyozov [8], coarsening processes have played prominent role in statistical [9] and biological physics [10], and in applied physical sciences [11-17]. Technologically significant thin films are no exception to this, as exemplified by growing microstructures seen in epitaxial growth [11,14].

A new venue in this area emerged from recent experimental revelations that long range de-wetting forces acting across thin films, such as the above discussed van der Waals interactions [18], may induce the formation of large clusters, i.e., tall multi-layer islands formed during high temperature annealing (relaxation) of thin films, [19]. Earlier experiments with Sn [13] and Mn films [15] on silicon evidence that the clusters grow in time by a coarsening process, with cluster linear size $\sim t^{1 / 4}$ (at long times $t$ ), as predicted by Chakraverty $[16,17]$. However, a more complex mode of cluster growth 
has been also seen, in solid Co films on sapphire $[7,20]$. In addition, an intriguing long lasting early-time cluster growth behavior, preceding the $t^{1 / 4}$ regime, has been seen in the experiments [13].

Motivated by the experiments, in this Thesis we elucidate the fundamentals of the non-equilibrium statistical mechanics of solid films coarsening within a unified film interface dynamics model explicitly incorporating de-wetting interactions. By analytic arguments and simulations of D+1 -dimensional films on D-dimensional substrates, we study the growth of clusters formed due to de-wetting interactions, such as the common van der Waals and other experimentally significant long range interactions discussed in the previous section. We find that the cluster height $H \sim t^{1 / 4}$, for any $D \geq 2$ (a superuniversal behavior), in accord with the experiments $[13,15]$ whence $D=2$. On the other hand, for $D<2$, the super-universality breaks down; $H \sim t^{\beta_{H}}$ with a D-dependent exponent $\beta_{H}=D /(2+3 D)$. These scaling laws hold at long times, for both short and long range de-wetting interaction. However, we reveal that long range (power-law) dewetting interactions introduce a long lasting early-time (cross-over) scaling behavior that is especially prominent for $D=2$ whence it yields effective cluster height growth exponent $\beta_{H}^{\text {eff }}>1 / 4$. This behavior persists over several decades of time and it is thus experimentally relevant. Such a pronounced cross-over behavior, with a $\beta_{H}^{\text {eff }}>1 / 4$, has been indeed seen in the experiments [13], and its physical origin has remained elusive to this date. In this Thesis, this long lasting cross-over phenomenon is explained as an effect of the ubiquitous long range de-wetting interactions we discussed in Sec. 1.1. We theoretically prove, by numerical simulations and analytic methods, that the long range nature of de-wetting forces indeed produces long lasting early-time departures from the asymptotic power laws, with the effective exponent $\beta_{H}^{\text {eff }}>1 / 4$ for $D=2$. Experimentally, such an effective early-time exponent, measured to be $\beta_{H}^{\text {eff }} \approx 0.33$, has been indeed reported for Sn clusters on silicon, Ref. [13]. This early-time cluster growth regime is shown here to be marked by a growing cluster height-to-width aspect ratio which slowly approaches the aspect ratio determined by the Young contact angle. In this respect, notable are the experiments of Ref. [7], in which the clusters are indeed 
seen to exhibit a growing cluster aspect ratio. For realistic de-wetting potentials, such as van der Waals and other long range interactions, these early-time crossover phenomena are found here to ubiquitously persist over several decades of time and they are thus experimentally significant.

The organization of this Thesis is as follows: In Chapter 2, we introduce the interface dynamics model and discuss its properties in detail. In particular, in Sec. 2.5, we discuss a simple kinetic theory of the coarsening dynamics of the clusters formed in our model. This discussion provides the values of the cluster growth exponents (such as the above mentioned exponent $\beta_{H}$ ) that are valid only in the long times limit. In this limit, the clusters can be described by the classical Young-Laplace model. In Chapter 3, we use numerical simulations of our interface dynamics model to explore the role played by the long-range de-wetting interactions on the character of the cluster coarsening dynamics. We find that these interactions produce significant early time departures from the asymptotic predictions based on the Young-Laplace model, as anticipated in this section. To address this phenomenon, in Chapter 4, we first provide a theoretical description of quasi-static (slowly evolving) clusters that goes beyond the classical Young-Laplace model. Next, in Chapter 5, we use this description to develop a powerful cluster dynamics theory capable to analytically explain the findings of our simulations. Finally, at the end we provide a summary and conclusion of this Thesis. We note that each chapter contains its own list of references. 


\subsection{References:}

[1] H.B.G. Casimir, Proc. K. Ned. Akad. 51, 79 (1948).

[2] E.M. Lifshitz, J. Exp. Theor. Phys. 29, 94 (1955).

[3] I.E. Dzyaloshinskii, E.M. Lifshits, and L.P. Pitaevskii, Sov. Phys. - JETP 37 (1960) 161

[4] J.N. Israelachvili, Intermolecular and Surface Forces (Academic, London, 1992).

[5] A. Bulgac and A. Wirzba, Phys. Rev. Lett. 87, 120404 (2001).

[6] A. Wirzba, A. Bulgac and P. Magierski, J. Phys. A: Math. Gen. 39, 6815 (2006).

[7] J. Espinosa, De-wetting of cobalt thin films on sapphire, Ph.D. Thesis, West Virginia University (2007), at http://wvuscholar.wvu.edu.

[8] I.M. Lifshits and V.V. Slyozov, Sov. Phys. JETP 35 (1959) 331.

[9] A.J. Bray, Adv. Phys. 34 (1994) 357.

[10] D. Moldovan and L. Golubović, Phys. Rev. Lett. 82 (1999) 2884; L. Golubović, D. Moldovan, and A. Peredera, Phys. Rev. Lett. 81 (1998) 3387.

[11] J. Krug, Adv. in Phys. 46 (1997) 139; Physica A 313 (2002) 47.

[12] J.W. Cahn and J.E. Hilliard, J. Chem. Phys. 28 (1958) 258.

[13] M. Zinke-Allmang and L.C. Feldman, Appl. Surf. Sci. 52 (1991) 357.

[14] M. Siegert, Phys. Rev. Lett. 81 (1998) 5481;

D. Moldovan and L. Golubović, Phys. Rev. E 61 (2000) 6190.

[15] M.R. Krause et al. , J. Vac. Sci. Technol. A 24 (2006) 1480.

[16] B.K. Chakraverty, J. Phys. Chem. Solids 28 (1967) 2401.

[17] K. Shorlin, S. Krylov, and M. Zinke-Allmang, Physica A 261 (1998) 248.

[18] P.G. de Gennes, Rev. Mod. Phys. 57 (1985) 827.

[19] C. Favezza, R. Kalynanaraman, and R. Sureshkumar, Nanotechnol. 17, (2006) 1536.

[20] J. Espinosa, H.T. Shi, and D. Lederman, J. Appl. Phys. 99 (2006) 023516; H.T. Shi, and D. Lederman, Phys. Rev. B 58 (1998) R1778. 


\section{Chapter 2}

\section{Interface Dynamics Model with De-wetting Interactions}

\subsection{The Model}

In this thesis we discuss a simple model for solid film interface dynamics respecting the conservation of the film volume, of the general form [1, 2],

$$
\frac{\partial h}{\partial t}=-\vec{\nabla} \cdot \vec{J}
$$

where $h(\vec{x}, t)$ is the film interface height over a D-dimensional base plane, $\vec{x}=\left(x_{1}, x_{2}, \ldots, x_{D}\right)$. In Eq. (1), $\vec{J}$ is the surface current density [1,2], given by the NernstEinstein equation,

$$
\vec{J}=-\Gamma \vec{\nabla} \mu=-\Gamma \Omega \vec{\nabla} P .
$$

Here, $\Gamma$ is a transport coefficient, and $\mu$ is chemical potential field; $\mu=\Omega P$, with $\Omega$, the atomic volume. As detailed in the Appendix of this chapter, within the small slope approximation (SSA), $m=|\vec{\nabla} h|<<1$, the pressure field $P(\vec{x}, t)$ in Eq. (2) is generated by the surface contribution to the film free energy, of the form [3],

$$
F=\int d^{D} x\left[\frac{\gamma}{2}(\vec{\nabla} h)^{2}+\Phi(h)\right]
$$


via the relation

$$
P(\vec{x}, t)=\frac{\delta F}{\delta h(x, t)}=-\gamma \vec{\nabla}^{2} h+\Phi^{\prime}(h)
$$

Above, $\gamma$ is the surface tension of the film at its top interface with vacuum, whereas $\Phi(h)$ is the de-wetting interaction potential. Within the SSA, the $\gamma$ is taken to be isotropic. As discussed in Ch. 1, physically interesting de-wetting potentials have the asymptotic form of a power law attraction,

$$
\Phi(h) \sim-\frac{1}{h^{\omega}}
$$

for large $h$; Fig. 1 .

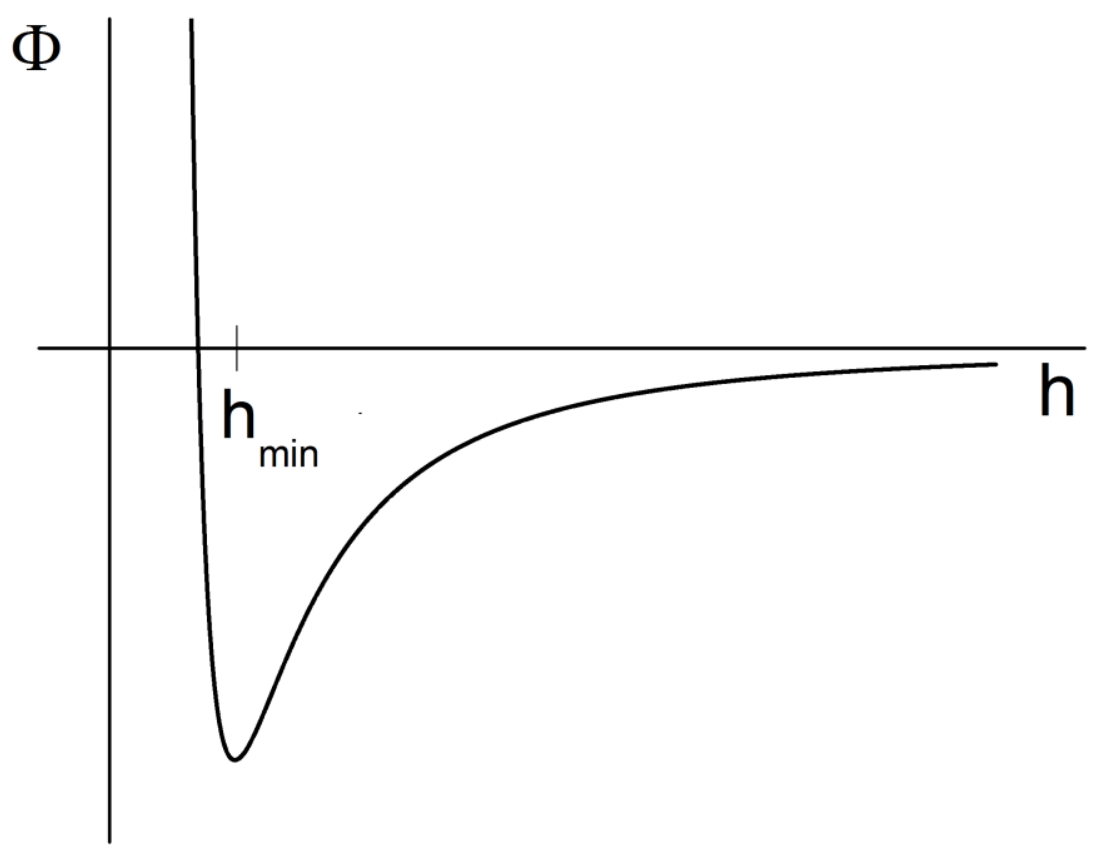

Fig. 1. De-wetting interaction potential causing the flat film instability. 
The common non-retarded van der Waals interactions correspond to the case $\omega=2$ of Eq. (4), [3]. The case $\omega=1$ may also be interesting [4]. Short range de-wetting interactions are regained in the large- $\omega$ limit; $\omega>>1$. By Eqs. (1-3),

$$
\frac{\partial h}{\partial t}=\Gamma \Omega \vec{\nabla}^{2} P=-\Gamma \Omega \vec{\nabla}^{2}\left[\gamma \vec{\nabla}^{2} h-\Phi^{\prime}(h)\right] .
$$

For $\Phi(h)=0$, Eq. (5) reduces to the classical Mullins model for solid interface relaxation by surface diffusion [1].

\subsection{Spinodal Instability}

The presence of the de-wetting potential $\Phi(h)$ induces an undulational (spinodal dewetting) instability of flat $(h=$ const.) films $[4,5]$. To demonstrate its presence in our model, consider a thin film of average thickness $h_{0}$, with a small fluctuation $\delta h(\vec{x}, t)$ at a time $t$ and position $\vec{x}$ :

$$
h(\vec{x}, t)=h_{0}+\delta h(\vec{x}, t)
$$

with $\delta h<<h_{0}$, as depicted in Fig. 2.

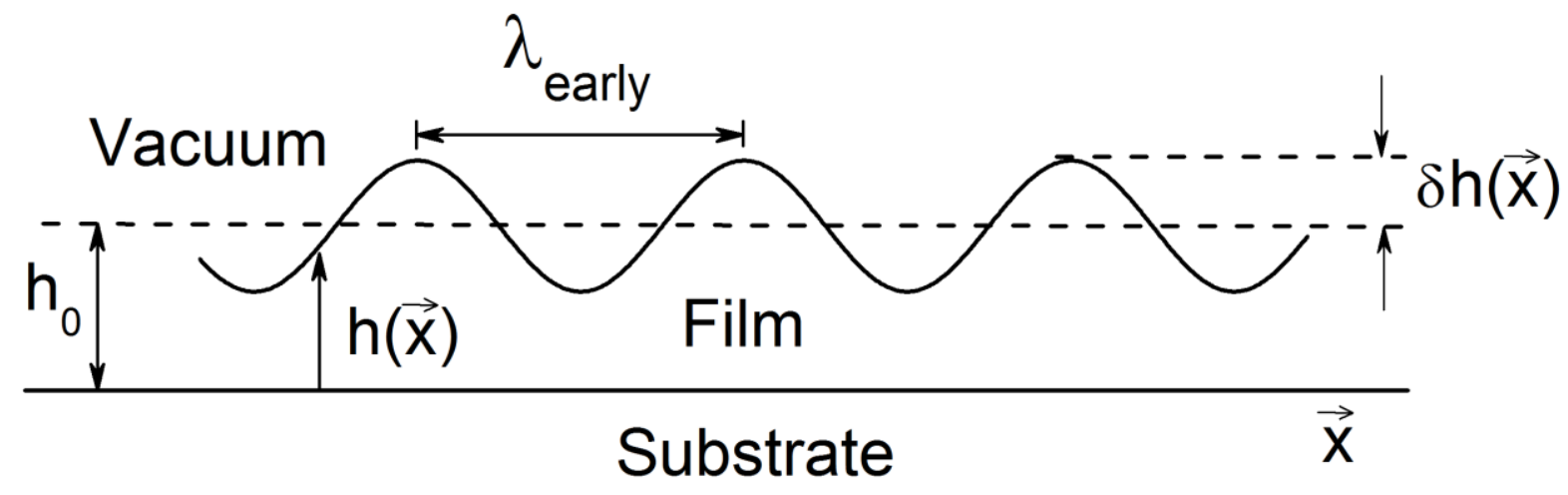

Fig. 2. Schematic view of small fluctuations $\delta h$ of the thin film surface around average thickness $h_{0}$. 
By expanding the interaction potential about the thickness $h_{0}$ we have

$$
\Phi^{\prime}(h)=\Phi^{\prime}\left(h_{0}\right)+\Phi^{\prime \prime}\left(h_{0}\right) \delta h,
$$

to the first order. Therefore, by Eqs. (5-7),

$$
\frac{\partial(\delta h)}{\partial t}=-\Gamma \Omega \vec{\nabla}^{2}\left[\gamma \vec{\nabla}^{2} \delta h-\Phi^{\prime \prime}\left(h_{0}\right) \delta h\right] .
$$

This linear PDF can be solved by

$$
\delta h(\vec{x}, t)=A_{\vec{q}}(t) \exp (i \vec{q} \vec{x}),
$$

where $A_{\vec{q}}$ is a time dependent amplitude of the film's fluctuation with the wavevector $\vec{q}$. By Eq. (9), the equation (8) reduces to

$$
\frac{d A_{\bar{q}}(t)}{d t}=G(q) A_{\vec{q}}(t) ，
$$

with the dispersion relation $G(q)$ given by

$$
G(q)=\Gamma \Omega \cdot\left(-\gamma q^{4}-q^{2} \Phi^{\prime \prime}\left(h_{0}\right)\right) .
$$

By Eq. (10), the time dependent amplitude $A_{\vec{q}}(t)$ is given by

$$
A_{\vec{q}}(t)=A_{\bar{q}}(0) \exp [G(q) t] .
$$

Eqs. (11)-(12) are essential for understanding the initiation of the thin film de-wetting process. If $\Phi^{\prime \prime}\left(h_{0}\right)>0$, the $G(q)$ is negative for any $\vec{q}$, and the fluctuations of the film about the initial thickness $h_{0}$ will smooth out. The film is thus stable. On the other hand, as depicted in Fig. 3, if $\Phi^{\prime \prime}\left(h_{0}\right)>0$, the $G(q)$ is positive in the range $|\vec{q}|<q_{c}$, with 


$$
q_{c}=\sqrt{\frac{-\Phi^{\prime \prime}\left(h_{0}\right)}{\gamma}} .
$$

The fluctuations with $|\vec{q}|<q_{c}$ grow exponentially in time, making the film interface unstable. Therefore, the condition for the film instability is :

$$
\Phi^{\prime \prime}\left(h_{0}\right)<0
$$

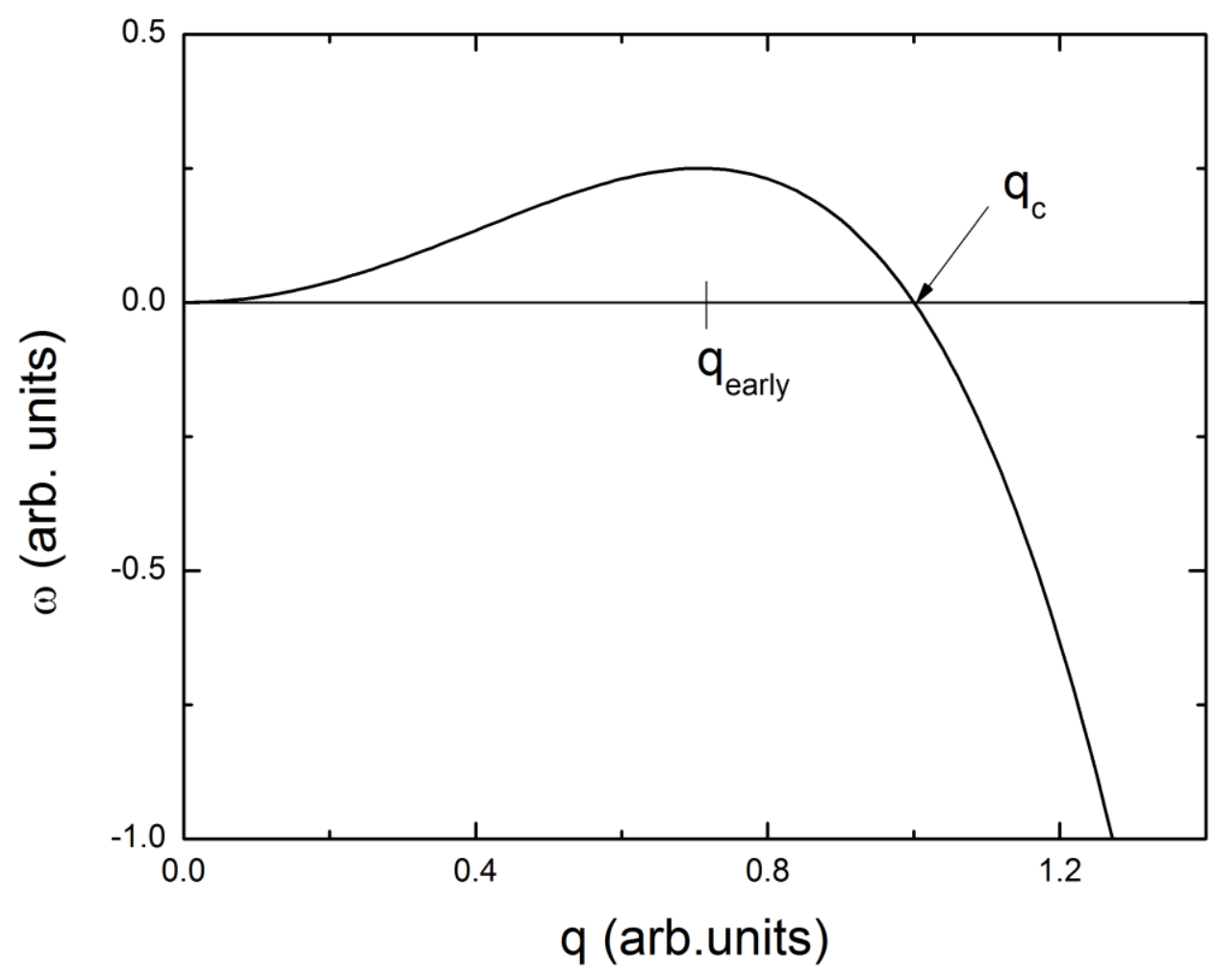

Fig. 3. Variation of the dispersion relation $G(q)$ as function of the magnitude of the wavevector $q$.

This instability initiates the de-wetting of the thin film. In this initiation, the dominant role is played by the fastest growing fluctuations, with the largest value of $G(q)$ 
corresponding to its maximum seen in Fig. 3. Their wave-vector $q_{\text {early }}$ can easily be evaluated from the condition

$$
\left.\frac{d \omega(q)}{d q}\right|_{q_{e a r l y}}=0
$$

Thus we obtain

$$
q_{\text {early }}=\sqrt{\frac{-\Phi^{\prime \prime}\left(h_{0}\right)}{2 \gamma}}=\frac{q_{c}}{\sqrt{2}},
$$

corresponding to the wavelength

$$
\lambda_{\text {early }}=\frac{2 \pi}{q_{\text {early }}}=2 \pi \sqrt{\frac{2 \gamma}{-\Phi^{\prime \prime}\left(h_{0}\right)}} .
$$

The corresponding value of the dispersion relation Eq. (11) is

$$
G\left(q_{\text {early }}\right)=\Gamma \Omega \cdot \frac{\left[\Phi^{\prime \prime}\left(h_{0}\right)\right]^{2}}{4 \gamma}
$$

The reciprocal of $G\left(q_{\text {early }}\right)$ is the characteristic time scale for the growth of the spinodal decomposition instability. We note that the equations (16-17) are general because they do not assume a certain shape of the interaction potential. The only essential condition is that $\Phi^{\prime \prime}\left(h_{0}\right)<0$. Notable feature of the wavelength $\lambda_{\text {early }}$ is that it depends on the initial thickness $h_{0}$ of the film.

Due to the spinodal instability, the thin film structures into a periodic like pattern with wavelength $\lambda_{\text {early }}$. As the film continues to evolve, it will subsequently form arrays of pits [see Fig. 5(a) from our simulations]. These arrays of pits are however unstable and subsequently transform into arrays of cluster [see Fig. 5(b) from our simulations]. The initial separation between these clusters is $\sim \lambda_{\text {early }}$. These clusters continue to grow via a coarsening process discussed in the following chapters. 


\subsection{Young Contact Angle}

Due to the presence of clusters, at long times the film partially "de-wets": Large slowly growing (quasi-static) clusters sitting on "dry" substrate area are formed, as seen in our simulations in $D=1$ and 2; see Figs. 4 and 5(b). The dry area, i.e., the substrate corresponds to the minimum $\Phi_{\min }$ of $\Phi(h)$, at $h_{\min }$ ( atomic size) in Fig. 1 . In view of this, the actual local film thicknes is given by the expression

$$
\tilde{h}(\vec{x}, t)=h(\vec{x}, t)-h_{\min }
$$

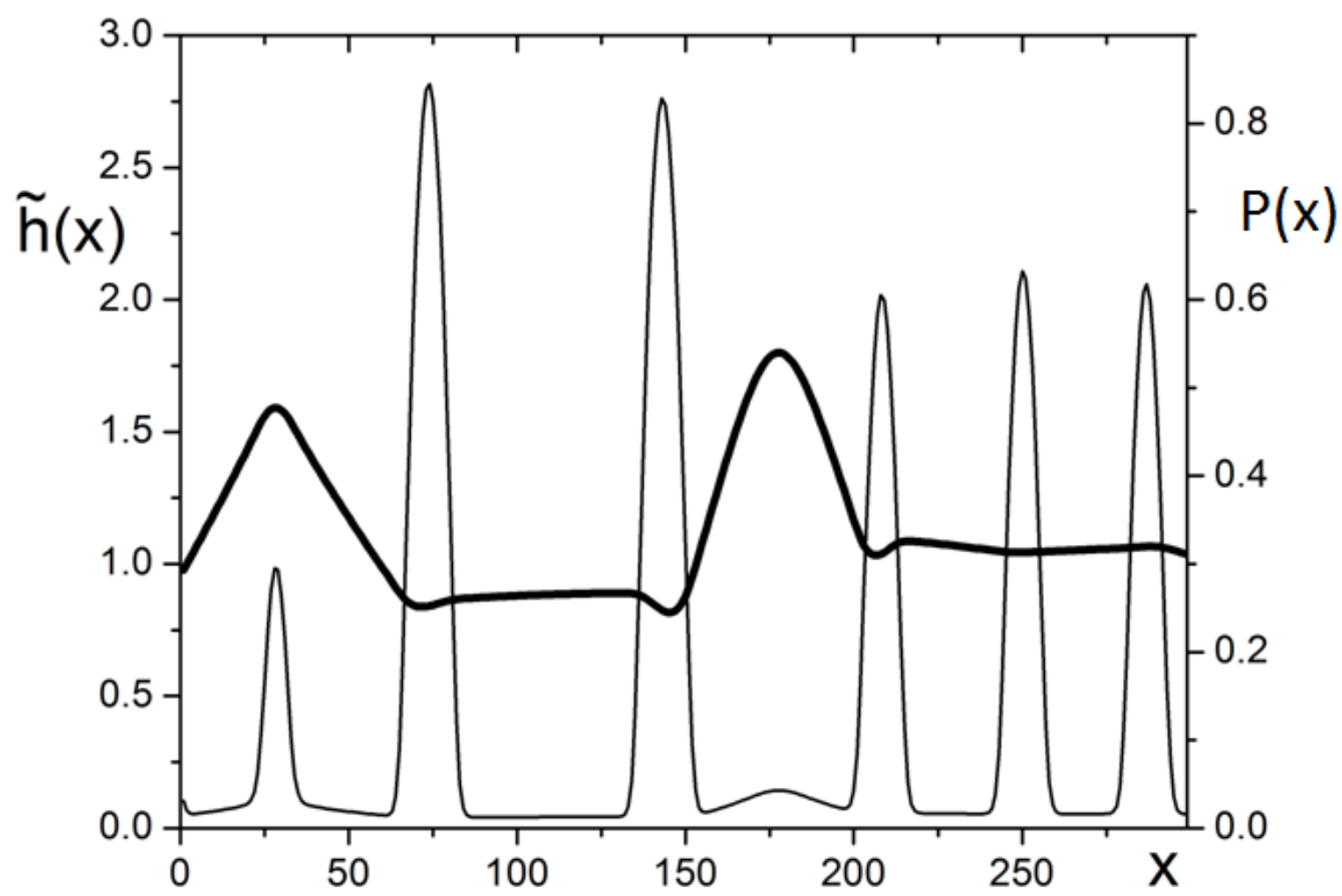

Fig. 4. A snapshot from our simulations: The interface profile $\tilde{h}(x, t)=h(x, t)-h_{\min }$ (thinner line) in $D=1$ and the corresponding field of pressure $P(x, t)$ (thicker line). 
In the limit of very large clusters, the cluster pressure $P \rightarrow 0$ [see Ch. 3]. In this limit, the maximum cluster slope [occurring close to cluster base in Fig. 5(b), at an inflection point of cluster radial profile] $m_{\max }(t)=\tan \left(\theta_{\max }\right)=|\vec{\nabla} h|_{\max }$ approaches the Young contact angle slope $m_{Y}=\tan \left(\theta_{Y}\right)$. The Young contact angle can be calculated for our model: By Eq. (3b), in the limit $P \rightarrow 0$ we obtain:

$$
-\gamma \vec{\nabla}^{2} h+\Phi^{\prime}(h)=0
$$
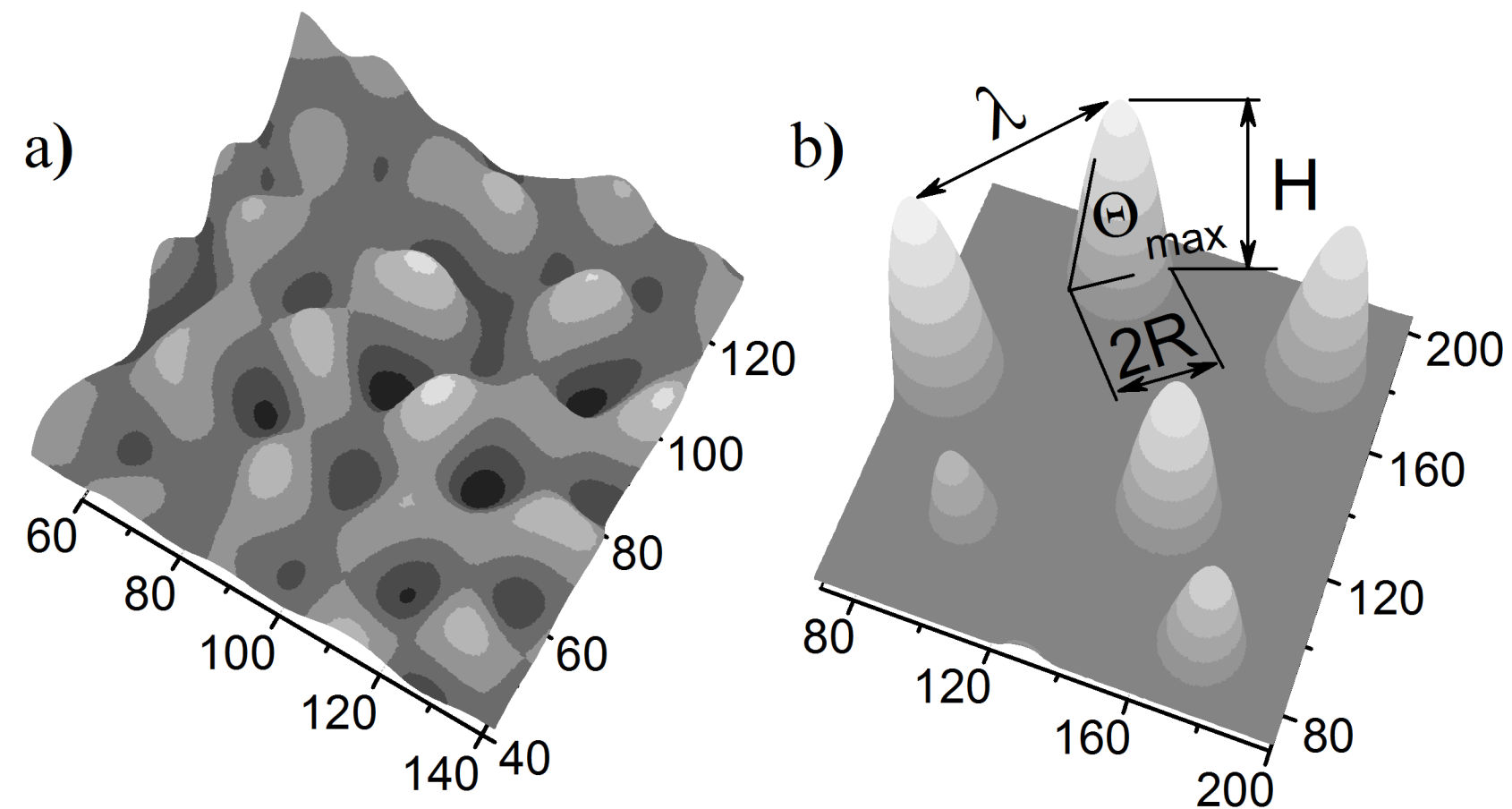

Fig. 5. Snapshots from our simulations: (a) The initial stage of the $D=2$ thin film interface, dominated by the formation of pits. Figure (b) exhibits the final stage of the $D=2$ thin film interface evolution, dominated by the presence of growing clusters. 
For example, for one dimensional case $(D=1)$, Eq. (18) reduces to

$$
\gamma \frac{d^{2} h}{d x^{2}}-\Phi^{\prime}(h)=0
$$

By the identities $\frac{d}{d x}\left(\frac{d h}{d x}\right)^{2}=2 \frac{d h}{d x} \frac{d^{2} h}{d x^{2}}$ and $\frac{d \Phi(h)}{d x}=\Phi^{\prime}(h) \frac{d h}{d x}$, Eq. (19) implies

$$
\frac{d}{d x}\left\lfloor\frac{\gamma}{2}\left(\frac{d h}{d x}\right)^{2}-\Phi(h)\right\rfloor=0,
$$

that is,

$$
\frac{\gamma}{2}\left(\frac{d h}{d x}\right)^{2}-\Phi(h)=C
$$

where $C$ is an integration constant. Equation (21) describes the interface profile around the "contact point" of the film with the "dry" substrate. On one side of the "contact point", the profile $h(x)$ asymptotically approaches $h_{\min }$, i.e., the local film thickness $\tilde{h}(\vec{x})=h(\vec{x})-h_{\min } \rightarrow 0$. In this limit, by Eq. (21) we obtain that $C=-\Phi\left(h_{\min }\right) \equiv-\Phi_{\min }$. With this condition, Eq. (21) reduces to

$$
\frac{\gamma}{2}\left(\frac{d h}{d x}\right)^{2}-\Phi(h)=-\Phi_{\min }
$$

On the other side of the contact point, $h \rightarrow \infty$ for $x \rightarrow \infty$. In this limit, $\Phi(h \rightarrow \infty) \rightarrow 0$. Thus, by Eq. (22), the interface slope $d h / d x$ approaches the Young angle slope given by

$$
m_{Y}=\tan \left(\theta_{Y}\right)=\sqrt{\frac{-2 \Phi_{\min }}{\gamma}} .
$$

The result (23) for the Young contact angle is general and applies in any $D$. To see this, consider, for example, the two dimensional case $(D=2)$. For a radially symmetric cluster, Eq. (3b) in polar coordinates reduces to 


$$
\gamma\left\lfloor\frac{d^{2} h}{d r^{2}}+\frac{1}{r} \frac{d h}{d r}\right\rfloor-\Phi^{\prime}(h)=-P .
$$

Around the contact point, we can expand $r=R+x$ with $x<<R$, where $R$ is the cluster base radius, as in Fig. 5(b). Thus, Eq. (24) becomes:

$$
\gamma\left\lfloor\frac{d^{2} h}{d x^{2}}+\frac{1}{R+x} \frac{d h}{d x}\right\rfloor-\Phi^{\prime}(h)=-P .
$$

Expanding, $\frac{1}{R+x}=\frac{1}{R}\left(1-\frac{x}{R}+\frac{x^{2}}{R^{2}}-\ldots\right)$ for $x<<R$, we obtain:

$$
\gamma \frac{d^{2} h}{d x^{2}}+\frac{\gamma}{R}\left(1-\frac{x}{R}+\frac{x^{2}}{R^{2}}-\ldots\right) \frac{d h}{d x}-\Phi^{\prime}(h)=-P .
$$

In the limit of large clusters $R \rightarrow \infty$, the cluster pressure $P \rightarrow 0$ (see Sec. 2.5 in the following). In this limit, the equation (26) reduces to Eq. (19) we had for the one dimensional case. Thus, the result for the contact angle slope $m_{Y}=\tan \left(\theta_{Y}\right)$ stated in Eq. (23) applies also for large clusters in $D=2$. 


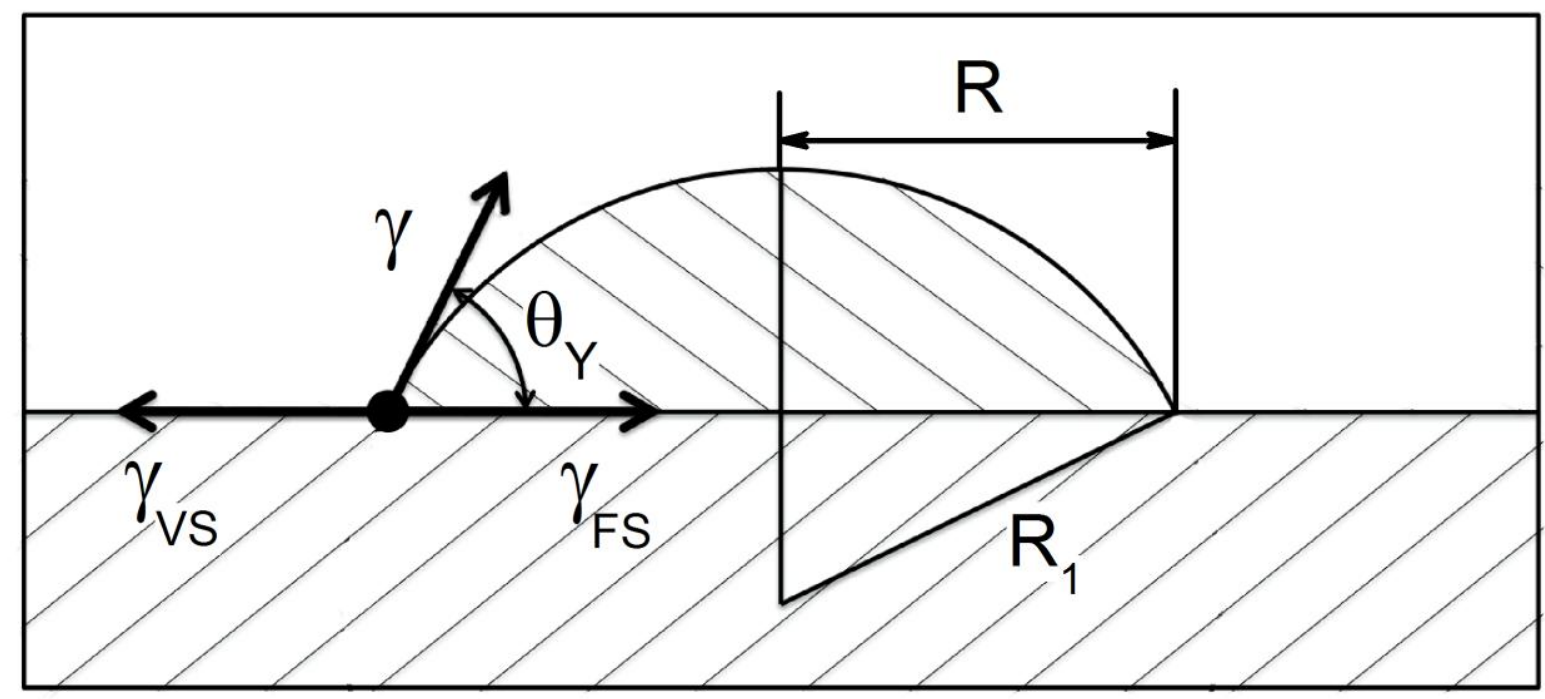

Fig. 6. Sketch of a cluster on top of a substrate depicting the Young-Dupré model of de-wetting ; $\gamma$, $\gamma_{F S}$ and $\gamma_{V S}$ are the interface energies at vacuum-film, film-substrate and vacuum-substrate interfaces, respectively ; $\theta_{Y}$ is the cluster-substrate contact angle and $R_{1}$ is the radius of the curvature of the cluster interface with vacuum whereas $R$ is the radius of the cluster circular base.

It is physically illuminating to note that, within the SSA, our equation (23) turns out to be equivalent to the standard Young-Dupré law,

$$
\gamma \cos \left(\theta_{Y}\right)+\gamma_{F S}=\gamma_{V S}
$$

for the cluster-substrate contact angle $\theta_{Y}$; see Fig. 6.

To see this equivalence, we note that the film-substrate surface tension $\gamma_{F S}$ and vacuum-substrate surface tension $\gamma_{V S}$ satisfy the relation

$$
\gamma_{V S}-\gamma_{F S}-\gamma=\Phi_{\min } \equiv \Phi\left(h_{\min }\right),
$$


suggested by de Gennes [3]. Within the SSA, $\cos \left(\theta_{Y}\right) \approx 1-\tan ^{2}\left(\theta_{Y}\right) / 2$, and, by Eq. (28), it is easy to see that Eqs. (23) and (27) are equivalent to each other. It should be stressed that this discussion proves the validity of the Young-Dupré law only in the limit of large clusters [recall of our discussions around Eq. (26)]. For a finite size cluster, the effects of de-wetting interactions become more significant and the Young-Dupré law is not applicable. This feature gives rise to the interesting phenomena we will reveal in the following chapters of this Thesis.

\subsection{Rescaled Model}

Motivated by Eq. (4) and by the above discussions, we simulated the model in Eq. (5) with

$$
\Phi^{\prime}(h)=\frac{A}{h^{\omega+1}}\left\lfloor 1-\left(\frac{h_{\min }}{h}\right)^{\omega}\right\rfloor,
$$

corresponding to the potential

$$
\Phi(h)=-\frac{A}{\omega h^{\omega}}\left\lfloor 1-\frac{1}{2}\left(\frac{h_{\min }}{h}\right)^{\omega}\right\rfloor,
$$

having minimum at $h_{\min }$, as in Fig. 1 . With this $\Phi(h)$, by a suitable rescaling of $(\vec{x}, t, h)$, the continuum model stated in Eqs. (5) and (29) can be made dimensionless (with $\Gamma \Omega=\gamma=A=1)$, of the form,

$$
\frac{\partial h}{\partial t}=\vec{\nabla}^{2} P=-\vec{\nabla}^{2}\left[\vec{\nabla}^{2} h-\Phi^{\prime}(h)\right]
$$

and

$$
\Phi^{\prime}(h)=\frac{1}{h^{\omega+1}}\left\lfloor 1-\left(\frac{h_{\min }}{h}\right)^{\omega}\right\rfloor .
$$


We chose the rescaling of the original model such that, in the rescaled model,

$$
h_{\min }=\left(\frac{\omega+2}{2(2 \omega+1)}\right)^{1 / \omega} .
$$

This choice is a matter of future convenience. [Other choices, such as $h_{\min }=1$, are also possible to achieve by the choice of rescaling. The choice in Eq. (33) is preferred because it gives a very simple form to the equation (39) discussed in the following.] By Eqs. (32) and (33), the rescaled model potential

$$
\Phi(h)=-\frac{1}{\omega h^{\omega}}\left\lfloor 1-\frac{1}{2}\left(\frac{h_{\min }}{h}\right)^{\omega}\right\rfloor,
$$

has a signle inflection point

$$
\Phi^{\prime \prime}\left(h_{\text {inf }}\right)=0,
$$

at the height

$$
h_{\mathrm{inf}}=\left(\frac{\omega+2}{2(\omega+1)}\right)^{1 / \omega} .
$$

For the initial $(t=0)$ thin film interface heights $\quad h(x t=0)=h_{0}$ that are above the inflection point, one has

$$
\Phi^{\prime \prime}\left(h_{0}\right)<0 ，
$$

Thus, by Eq. (14), such films are unstable and will undergo spinodal de-wetting. Interestingly, among these unstable films there is one which is the most unstable. Indeed, Eq. (17) shows that by maximizing the magnitude of $\Phi "\left(h_{0}\right)$ one can minimize the time scale of the instability, i.e., maximize its rate. Thus, this maximization corresponds to the condition,

$$
\Phi^{\prime \prime \prime}\left(h_{0}\right)=0,
$$


for the initial film height. By Eqs. (32) and (36), it is easy to show that the Eq. (38) is solved by

$$
h_{0}=1 \text {. }
$$

Motivated by this feature, in our simulations of the rescaled model, we set the initial height to be

$$
h(\vec{x}, t=0)=h_{0}=1+\text { a small random field, }
$$

to initiate the spinodal de-wetting instability. Such very thin films undergo the fastest possible spinodal instability and quickly pass from the initial instability time stage to the stage characterized by cluster formation. It should be stressed that the initial condition in Eq. (40) was motivated by practical reasons, to avoid too much of our computation times spent on the relatively simple instability time stage. Our primary interest is in the post-instability stage when the clusters appear and grow. In Figs. 5 (a, b) , 7 and 8 we give views of growing clusters from our simulations discussed in the following chapters of this thesis. 


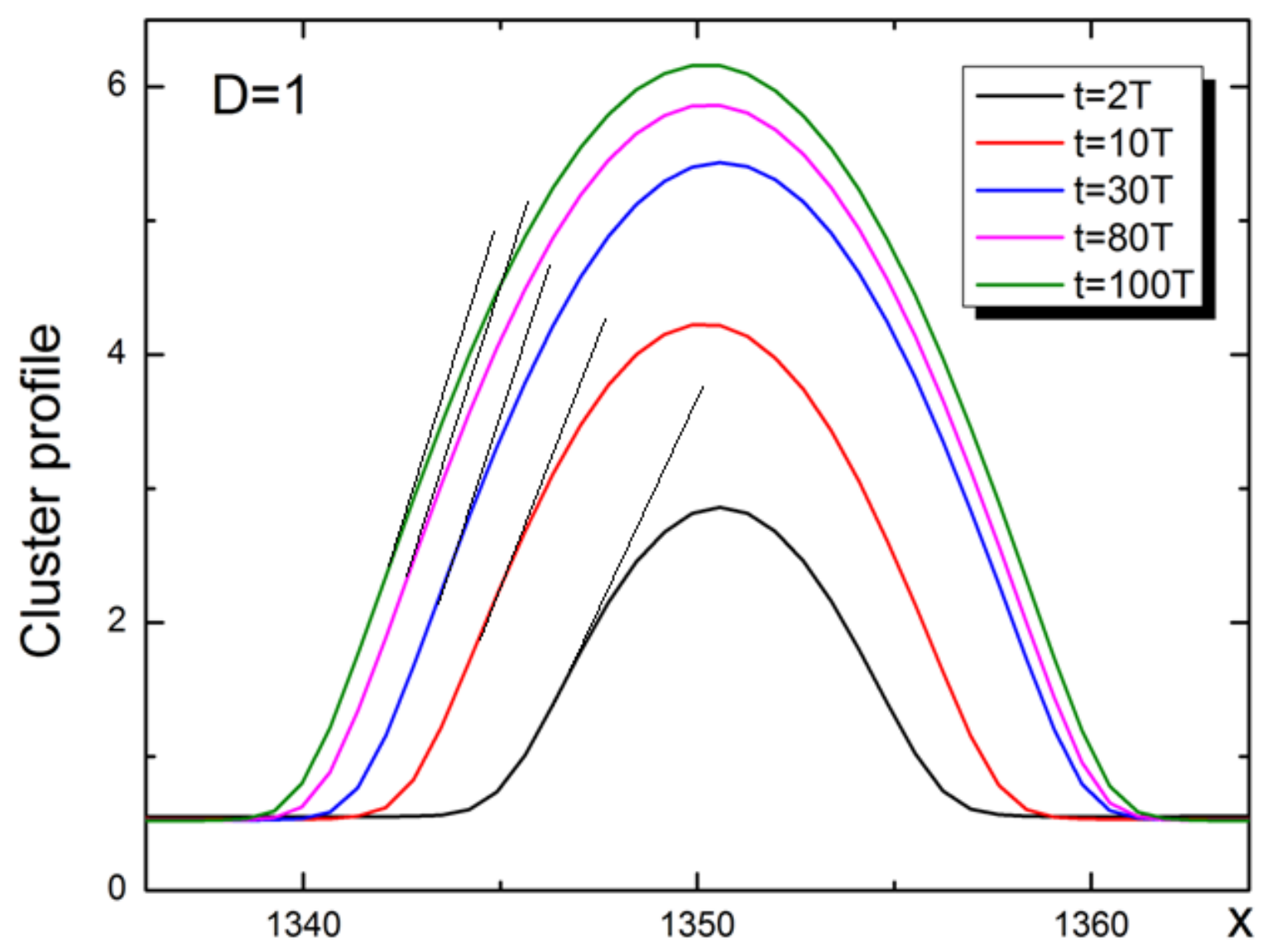

Fig. 7. Interface height plots showing the formation of a slowly growing cluster for $D=1$ and $\omega=1$, from our simulation of the rescaled model with dimensionless $t, \vec{x}$ and $h$. Here, $T$ is a characteristic time-scale at which the well structured clusters are seen for the first time. In the figure, we indicate tangents drawn at inflection points of the cluster profiles. Note that the tangent slope slowly grows in time. 


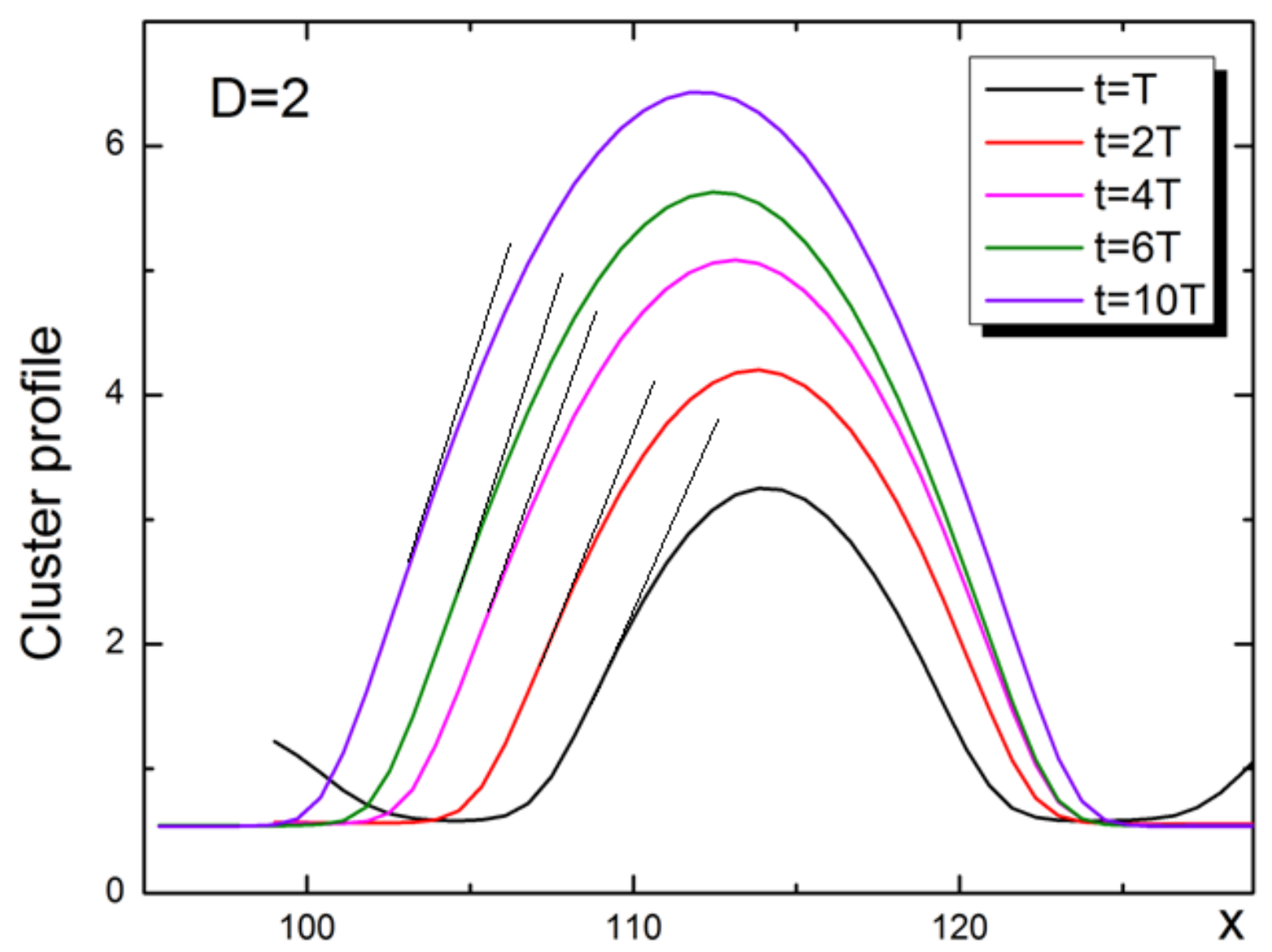

Fig. 8. Interface height plots showing the formation of a slowly growing cluster for $D=2$ and $\omega=1$, from our simulation of the rescaled model with dimensionless $t, \vec{x}$ and $h$ [as in Fig. 5(b)]. Here, $T$ is a characteristic time-scale at which the well structured clusters are seen for the first time. It corresponds to the crossover from pits arrays [Fig. 5(a)] to cluster dominated surface morphology [Fig. $5(b)]$. In the figure, we indicate tangents drawn at inflection points of the cluster profiles. Note that the tangent slope slowly grows in time. 
At the minimum, the rescaled potential in Eq. (34) has the value

$$
\Phi_{\text {min }}=\Phi\left(h_{\text {min }}\right)=-\frac{2 \omega+1}{\omega(\omega+2)} .
$$

Thus, by Eqs. (41) and (23) [applied to the rescaled model with $A=\gamma=1$ ], the Young angle slope for the rescaled model Eqs. (31-33) has the value,

$$
m_{Y}=\tan \left(\theta_{Y}\right)=\sqrt{\frac{2(2 \omega+1)}{\omega(\omega+2)}} .
$$

As discussed in Sec. 2.3, this result applies in the limit of large clusters. For a finite size cluster, the corresponding quantity is the maximum cluster slope [occurring close to cluster base in Fig. 5(b), at an inflection point of cluster radial profile] $m_{\max }(t)=\tan \left(\theta_{\max }\right)=|\vec{\nabla} h|_{\max }$. This slope is expected to approach the Young contact angle

slope $m_{Y}=\tan \left(\theta_{Y}\right)$ in the limit of large clusters. This feature is illustrated in Figs. 7 and 8 , in which we display the tangents drawn at the inflection points. One can see that slope of these tangents indeed slowly grows as the cluster grows. This phenomenon will play a significant role in the discussions made in the following chapters.

\subsection{Cluster Coarsening Dynamics in the Young-Laplace Limit}

In this section we will elucidate the cluster formation and the laws of cluster coarsening growth by a simple kinetic scaling theory. Of direct interest is the case $D=2$. We will however consider these phenomena in a general number of dimensions. Thus, in the following, we are looking at $D+1$ dimensional clusters on a $D$ dimensional substrate. The pressure field is nearly uniform within a cluster, $P(\vec{x}, t) \approx P_{\text {cluster }}$, with each cluster having a different pressure; see Fig. 4 from our simulations. In this section, we will consider clusters in the Young-Laplace limit. In this limit clusters are large and the cluster radial profile has the simple form 


$$
h(r) \approx H\left\lfloor 1-\left(\frac{r}{R}\right)^{2}\right\rfloor,
$$

for $r<R=$ cluster base radius, while

$$
P_{\text {cluster }} \approx \frac{2 D \gamma \cdot H}{R^{2}}
$$

The equations (43) and (44) are easily obtained by ignoring the potential $\Phi(h)$ in the equation 3(b). This reduces the equation to the simple Poisson equation

$$
P_{\text {cluster }} \approx-\gamma \nabla^{2} h
$$

spelling the classical Laplace relation between the pressure and the interface curvature. The potential $\Phi(h)$ however does become important for $r \approx R$ where the cluster slope assumes the value of the Young angle slope Eq. (23). Thus, by Eq. (43)

$$
m_{Y}=\tan \left(\theta_{Y}\right)=\left|h^{\prime}(R)\right| \approx \frac{2 H}{R} .
$$

By Eqs. (46) and (44), the cluster pressure has the form

$$
P_{\text {cluster }} \approx \frac{D \gamma m_{Y}^{2}}{2 H} \approx \frac{D \gamma m_{Y}}{R}
$$

valid in the Young-Laplace limit. By Eq. (1), the cluster volume $V_{\text {cluster }}$ changes in time according to the equation

$$
\frac{d}{d t} V_{\text {cluster }}=-I
$$

with, by Eq. (43),

$$
V_{\text {cluster }}=S(D) \int_{0}^{R} d r r^{D-1} h(r)=\frac{2 S(D)}{D(D+2)} H R^{D} .
$$


Here $S(D)$ is the surface area of the D-dimensional unit sphere, given by the expression,

$$
S(D)=\frac{(2 \pi)^{D / 2} 2^{1-D / 2}}{\operatorname{Gamma}(D / 2)},
$$

where Gamma stands for the ordinary gamma-function (here, of $D / 2$ ). Note that, for an integer valued $D$, the Eq. (50) reproduces the familiar results such as $S(D=3)=4 \pi$, $S(D=2)=2 \pi$, and $S(D=1)=2$. However, the above equation for $S(D)$, as well as our other discussions here, remain well defined even for non-integer $D$. The current $I$ in Eq. (48) is, by Eq. (1), the flux of the surface current density $\vec{J}$ through the cluster base boundary being the surface of D-dimensional sphere with the radius $=R$. By Eqs. (1) and (2) with $\partial h / \partial t \approx 0$, one has,

$$
-\vec{\nabla} \cdot \vec{J}=\Gamma \Omega \nabla^{2} P=0
$$

outside of the slowly evolving (quasi-static) clusters. Assuming here a radially symmetric solution, we obtain,

$$
\begin{aligned}
& J(r)=\frac{I}{S(D) r^{D-1}}=-\Gamma \Omega \frac{d P}{d r} \\
& \frac{d I}{d r}=0
\end{aligned}
$$

outside of a slowly evolving (quasi-static) cluster, that is, for $r$ in the range $R<r<\lambda$. Here, $\lambda$ is a characteristic distance corresponding to inter-cluster separation, see Fig. 5 (b). By integrating Eq. (51) over $r$, in the range $R<r<\lambda$

$$
P(R)-P(\lambda)=\frac{F_{D}(\lambda, R)}{S(D) \Gamma \Omega} I
$$

Here, $F_{D}(\lambda, R)=\int_{R}^{\lambda} d r / r^{D-1}$, and thus

$$
F_{D}(\lambda, R)=\frac{\lambda^{2-D}-R^{2-D}}{2-D}
$$

for $D \neq 2$, whereas for $D=2$ we have, 


$$
F_{D=2}(\lambda, R)=\ln \left(\frac{\lambda}{R}\right)
$$

Eq. (52) can be solved for the current $I$,

$$
I=S(D) \Gamma \Omega \frac{P(R)-P(\lambda)}{F_{D}(\lambda, R)} .
$$

The clusters are poly-disperse in sizes, with smaller than average clusters [with, by Eq. (47), higher pressure] decaying by expelling their material into larger clusters [with, by Eq. (47), lower pressure]; see Figs. 4 from our simulations. This coarsening yields a steady increase of average cluster height $H(t)$ and cluster base radius $R(t)$. The coarsening cluster growth is governed by the decay of the clusters that are comparable yet smaller than the average cluster size. For such a cluster $P_{\text {cluster }} \approx P(R)>P(\lambda)=$ the pressure of the nearest-neighbor (larger) cluster; see Fig. 4 from our simulations. Thus, by Eq. $\left(52^{\prime}\right)$,

$$
I \sim S(D) \Gamma \Omega \frac{P_{\text {cluster }}}{F_{D}(\lambda, R)},
$$

In the Young-Laplace limit, the result in Eq. (47) applies. With it, Eq. (55) yields,

$$
I \sim \frac{\kappa m_{Y}}{R F_{D}(\lambda, R)}
$$

with $\kappa=\Gamma \Omega \gamma$.

We note that the volume conservation law in Eq. (1) ensures that $V_{\text {cluster }}=$ the film volume initially (at $t=0$ ) covering the base area $\sim \lambda^{D}$, i.e., by Eq. (49),

$$
H R^{D} \sim h_{0} \lambda^{D}
$$

with $h_{0}$, the initial film thickness. By Eqs. (46) and (56),

$$
\lambda \sim\left(m_{Y} / h_{0}\right)^{1 / D} R^{1+1 / D}
$$

Note that by Eq. (57)

$$
\frac{\lambda}{R} \sim\left(\frac{m_{Y} R}{h_{0}}\right)^{1 / D} \sim\left(\frac{H}{h_{0}}\right)^{1 / D}>>1,
$$

for large clusters with $H>>h_{0}$. 
The differential equation (48), in combination with Eqs. (46), (49), (55'), and (57), can be easily integrated to find the time-scale of the cluster decay, with the result

$$
t \sim R^{D+2} F_{D}(\lambda, R) / \kappa .
$$

The cluster decay time-scale $\sim$ evolution time [10], so Eqs. (57) and (58) directly yield the desired coarsening laws for the growth of $R, H \sim m_{Y} R$, and $\lambda \sim R^{1+1 / D}$.

Case $D>2$ : As $\lambda>>R$, for $D>2$, one has, by Eq. (53), $F_{D}(\lambda, R) \sim R^{2-D}$. So, for $t \rightarrow \infty$, by Eqs. (58) and (46) one has

$$
H / m_{Y} \sim R \sim(\kappa t)^{1 / 4},
$$

for any $D>2$.

Case $D=2$ : For this case, $F_{D=2}(\lambda, R)=\ln (\lambda / R)$, so for $t \rightarrow \infty$, the Eqs. $\left(57^{\prime}\right)$ and (58) yield

$$
t \sim R^{4} \ln \left(R / R_{0}\right) / \kappa,
$$

with $R_{0} \sim h_{0} / m_{Y}$. By iteratively solving Eq. (60) for $R(t)$, we obtain

$$
H / m_{Y} \sim R \sim\left[\ln \left(t / t_{0}\right)\right]^{-1 / 4}(\kappa t)^{1 / 4},
$$

with $t_{0} \sim R_{0}^{4} / \kappa$, for $D=2$ and $t \rightarrow \infty$. Thus, up to a very slowly varying logarithmic pre-factor, one has $H \sim R \sim t^{1 / 4}$ for $t \rightarrow \infty$, in accord with our $D=2$ simulations [discussed in chapter 3] and the experiments [11,12].

Case $D<2$ : As $\lambda>>R$, for $D<2$ one has, by Eqs. (53) and (57), $F_{D}(\lambda, R) \sim \lambda^{2-D} \sim\left[\left(m_{Y} / h_{0}\right)^{1 / D} R^{1+1 / D}\right]^{2-D}$, so by Eq. (58), 


$$
t \sim m_{Y}^{(2-D) / D} R^{(2+3 D) / D} h_{0}^{-(2-D) / D} / \kappa .
$$

By Eq. (62), for $D<2$,

$$
H / m_{Y} \sim R \sim t^{D /(2+3 D)},
$$

for $t \rightarrow \infty$ whence $m_{\max } \rightarrow m_{Y}$. For $D=1$, Eq. (63) yields

$$
H / m_{Y} \sim R \sim t^{1 / 5} \text { for } t \rightarrow \infty
$$

Let us summarize the results of this section. At long times, i.e., in the YoungLaplace limit, we find that the cluster height $H \sim t^{1 / 4}$, for any $D \geq 2$ (a super-universal behavior), in accord with the experiments [11,12] whence $D=2$. In $D=2$ however, in addition to the power law, there is also a slowly changing logarithmic correction displayed in Eq. (61). On the other hand, for $D<2$, the super-universality breaks down; $H \sim t^{\beta_{H}}$ with a D-dependent exponent $\beta_{H}=D /(2+3 D)$. These scaling laws hold at long times, and they are insensitive to the actual form of the de-wetting potential such the value of the exponent $\omega$ in Eq. (4)]. It is interesting to note that our result in Eq. (64) for the special case $D=1$, coincides with the result obtained for a different model in Ref. [13]. The model in Ref. [13] actually describes coarsening of liquid films, in which case the transport coefficient $\Gamma$ depends on the interface height, rather than being constant as in our model for solid thin films. We also note that the existence of the logarithmic correction in $D=2$ has been noted (in passing) in Ref. [14], for the case of liquid films coarsening. However, unlike to our explicit result in Eq. (61), Ref. [14] does not provide any explicit formula (equation) giving the actual form of the logarithmic correction, that exists in $D=2$. We also note that the above discussed breakdown of super-universality was noted before in Ref. [14], for the case of liquid films. However, it has not been noted 
before that this breakdown of super-universality occurs only for $\mathrm{D}<2$, as shown by our discussions above.

For the future purposes of Chs. 4 and 5, it will be convenient to express the quasi-static cluster pressure $P$, height $H$, and base radius $R$, as functions of the cluster volume $V$. This can be easily done in the Young-Laplace limit, by a straightforward application of the Eqs. (44), (46), (47), and (49), yielding,

$$
H_{\text {stat }}^{\text {Young }}(V)=C_{H} V^{1 / 3}, \quad R_{\text {stat }}^{\text {Young }}(V)=C_{R} V^{1 / 3}, \quad P_{\text {stat }}^{\text {Young }}(V)=C_{P} V^{-1 / 3},
$$

for the physically interesting case $D=2$. In Eq. (65),

$$
C_{H}=(2 \pi)^{-1 / 3} m_{Y}^{2 / 3}, \quad C_{R}=(4 / \pi)^{1 / 3} m_{Y}^{-1 / 3}, \quad C_{P}=\gamma(2 \pi)^{1 / 3} m_{Y}^{4 / 3} .
$$

For example, for our rescaled model with $\gamma=1$, by Eqs. (66) and (42),

$$
\begin{aligned}
& C_{H}=(2 \pi)^{-1 / 3}\left(\frac{2(2 \omega+1)}{\omega(\omega+2)}\right)^{1 / 3}, \\
& C_{R}=(4 / \pi)^{1 / 3}\left(\frac{2(2 \omega+1)}{\omega(\omega+2)}\right)^{-1 / 6}, \\
& C_{P}=(2 \pi)^{1 / 3}\left(\frac{2(2 \omega+1)}{\omega(\omega+2)}\right)^{2 / 3} .
\end{aligned}
$$




\subsection{Appendix}

In this appendix we present a more detailed derivation of the theoretical model for solid film interface dynamics introduced in section 2.1.

Our model assumes the absence of evaporation and re-condensation process. Thus the thin film volume $V$ is conserved

$$
V(t)=\int d^{D} x h(\vec{x}, t)=\text { constant }
$$

The net free energy $F$ of the film depends on the interfacial height $h(\vec{x}, t)$ and contains three contributions:

$$
F=F_{b u l k}+F_{\text {surf }}+F_{\mathrm{int}}
$$

The first term in (A2) is the extensive, bulk contribution to the film free energy which is proportional to the film volume. This volume is conserved, so the first term in (A2) can not affect the interface dynamics and it can thus be dropped. The second term in (A2), $F_{\text {surf }}$ is the surface contribution to the free energy which is proportional to the surface

area, the proportionality constant being the surface tension of the film at its top interface with vacuum, $\gamma$. Hence,

$$
F_{\text {surf }}=\gamma \int d^{D} x \sqrt{1+|\vec{\nabla} h|^{2}} .
$$

In the small slope approximation, $m=|\vec{\nabla} h|<<1$, Eq. (A3) becomes:

$$
F_{\text {surf }}=\gamma A_{B}+\int d^{D} x \frac{\gamma}{2}(\vec{\nabla} h)^{2}
$$

where $A_{B}$ is the base plane area. Finally, the third term of Eq. (A2), $F_{\text {int }}$ is the interaction contribution to the free energy arising from the de-wetting potential $\Phi(h)$ 


$$
F_{\text {int }}=\int d^{D} x \Phi(h) .
$$

Thus, by dropping all constant terms, the film free energy (A2) can be expressed as in Eq. (3a), i.e.

$$
F=\int d^{D} x\left[\frac{\gamma}{2}(\vec{\nabla} h)^{2}+\Phi(h)\right]
$$

The variation of this free energy upon a small change $\delta h(x)$ of the interface height is

$$
\delta F=\int d^{D} x\left[-\gamma\left(\vec{\nabla}^{2} h\right)+\Phi^{\prime}(h)\right] \delta h .
$$

By the standard definition of the functional derivative, from Eq. (A6), we regain the pressure field $P(\vec{x}, t)$ introduced in Eq. $(3 b)$, i.e.

$$
P(\vec{x}, t)=\frac{\delta F}{\delta h(x, t)}=-\gamma \vec{\nabla}^{2} h+\Phi^{\prime}(h) .
$$

Thus, the Eq. (A6) can be written shortly as

$$
\delta F=\int d^{D} x P(x, t) \delta h(x) .
$$

In general, the chemical potential $\mu$ is the change of the free energy when the number of atoms of the film increases by one, say, by adding one more atom to its surface at the location $x_{0}$, as conceptualized in Figs. 9(a , b): $\mu=F(N+1)-F(N)=\delta F \quad(N$ being the number of atoms of the film). Thus, by Eq. (A6"), the chemical potential field is given by:

$$
\mu\left(x_{0}, t\right)=\delta F=\int d^{D} x P(x, t) \delta h\left(x, x_{0}\right),
$$

where $\delta h\left(x, x_{0}\right)$ is the change of the interface height profile corresponding to adding one atom at the location $x_{0}$. By assuming that the pressure field changes slowly over the atom size, Eq. (A7) yields 


$$
\mu\left(x_{0}, t\right)=P\left(x_{0}, t\right) \int d^{D} x \quad \delta h\left(x, x_{0}\right)=P\left(x_{0}, t\right) \Omega
$$

where $\Omega$ is the atomic volume,

$$
\Omega=\int d^{D} x \delta h\left(\vec{x}, x_{0}\right)
$$

Thus, by Eq. (A8) we obtain

$$
\mu(x, t)=\Omega \cdot P(x, t)=\Omega \cdot\left[-\gamma \vec{\nabla}^{2} h+\Phi^{\prime}(h)\right] .
$$

as noted below Eq. (2). 
a)

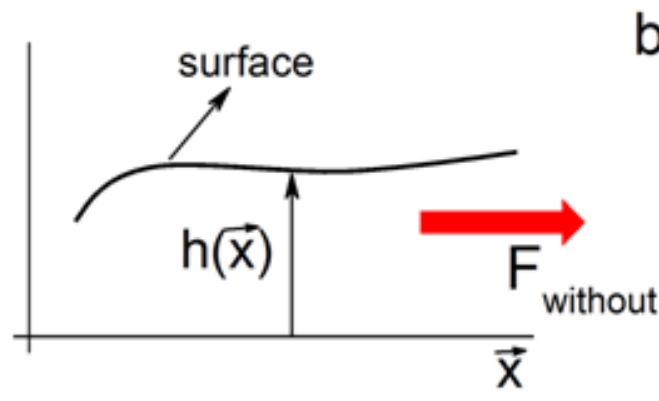

c)

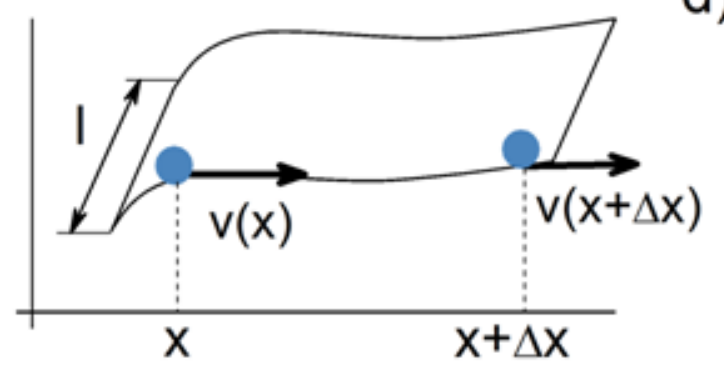

b)

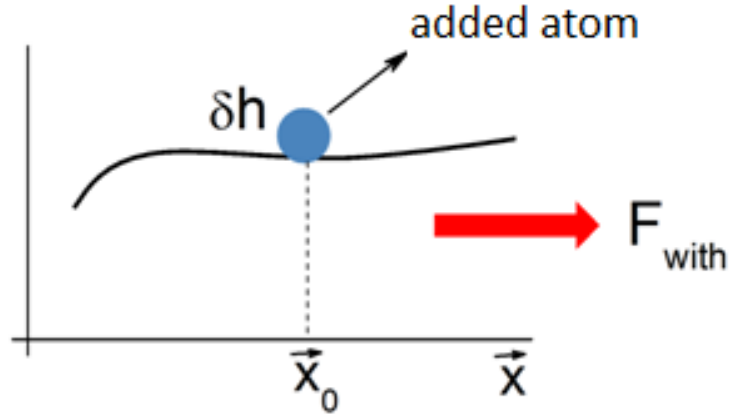

d)

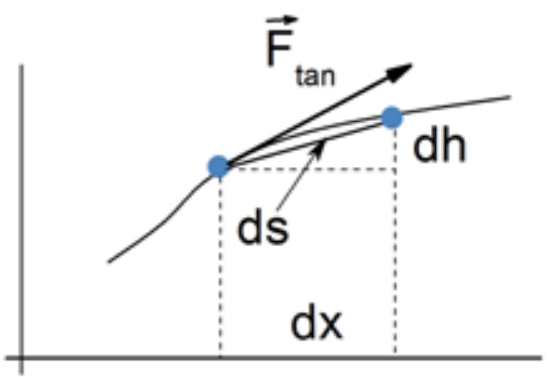

Fig. 9. (a) Schematic representation of a configuration with no atom added on the surface. The free energy of this configuration is labeled as $F_{\text {without }}$. In (b) we represent the configuration with the atom added on the surface at position $\vec{x}_{0}$, causing the interface height change $\delta h$. The free energy of this configuration is labeled as $F_{\text {with }}$. The difference of the free energy between these two configurations is the chemical potential field: $\mu\left(x_{0}\right)=F_{\text {with }}-F_{\text {without }}$. In figure (c) we represent schematically the surface atoms and $x$-components of their velocities at positions $x$ and $x+\Delta x$, respectively. Figure (d) shows the tangential force causing drift of surface atoms.

The chemical potential field in Eq. (A10) is an effective potential acting on the atoms that are free to diffuse along the film surface. Its -gradient (tangential force) will thus cause a drifting motion of these diffusing atoms. To proceed, for simplicity, let us consider a surface which height changes along one $(x)$ direction only; see Fig. 9(c). During the time interval $d t$, the film volume between $x$ and $x+\Delta x$ in Fig. 9(c) changes by the amount 


$$
l v(x) d t n_{0} \Omega-l v(x+\Delta x) d t n_{0} \Omega=d\left(\int d x h(x, t) l\right)
$$

The Eq. (A11) states that this volume changes due to the net influx of the drifting surface atoms. In Eq. (A11), $n_{0}$ is the surface density of the diffusing ("surface active") atoms, $\Omega$ the atomic volume, $v(x)$ and $v(x+\Delta x)$ are the drift velocities of the diffusing atoms at positions $x$ and $x+\Delta x$, respectively. Using the identity

$$
v(x+\Delta x)-v(x)=\int_{x}^{x+\Delta x} d x^{\prime} \frac{\partial v}{\partial x^{\prime}},
$$

by Eq. (A11) we obtain:

$$
-n_{0} \Omega \int_{x}^{x+\Delta x} d x^{\prime} \frac{\partial v}{\partial x^{\prime}}=\int_{x}^{x+\Delta x} d x^{\prime} \frac{\partial h\left(x^{\prime}, t\right)}{\partial t}
$$

Therefore,

$$
\frac{\partial h}{\partial t}=-n_{0} \Omega \frac{\partial v}{\partial x}
$$

The tangential force $F_{\text {tan }}$ acting on a surface atom is proportional to its velocity,

$$
v(x)=\alpha F_{\tan }(x)
$$

where $\alpha$ is a mobility constant. Therefore, Eq. (A13) becomes:

$$
\frac{\partial h}{\partial t}=-n_{0} \Omega \alpha \frac{\partial F_{\tan }(x)}{\partial x}
$$

The tangential force is given by the -gradient of the chemical potential along the arclength in Fig. 9(d),

$$
F_{\mathrm{tan}}=-\frac{\partial \mu}{\partial s}=-\frac{1}{\sqrt{1+\left(\frac{\partial h}{\partial x}\right)^{2}}} \cdot \frac{\partial \mu}{\partial x} \approx-\frac{\partial \mu}{\partial x}
$$


within the small slope approximation. Thus, by Eqs. (A14) , (A15) and (A10),

$$
\frac{\partial h}{\partial t}=n_{0} \Omega \alpha \frac{\partial^{2} \mu}{\partial x^{2}}=n_{0} \Omega \alpha \cdot \Omega \frac{\partial^{2} P}{\partial x^{2}} .
$$

By Eqs. (A16) and (A6'), we regain Eq. (5) i.e.

$$
\frac{\partial h}{\partial t}=\Gamma \Omega \vec{\nabla}^{2} P=-\Gamma \Omega \vec{\nabla}^{2}\left[\gamma \vec{\nabla}^{2} h-\Phi^{\prime}(h)\right],
$$

where

$$
\Gamma=n_{0} \Omega \alpha,
$$

is a transport coefficient. We recall the Eq. (A17), i.e., Eq. (5) is equivalent to the conservation law in Eq. (1), with the $\vec{J}$ given by

$$
\vec{J}=-\Gamma \vec{\nabla} \mu=-\Omega \Gamma \vec{\nabla} P=\Omega \Gamma \vec{\nabla}\left[\gamma \vec{\nabla}^{2} h-\Phi^{\prime}(h)\right],
$$

as stated in Eq. (2) . 


\subsection{References}

[1] W.W. Mullins, in Metal Surfaces, American Society of Metals, Metals Park, Ohio (1963) p. 17 .

[2] J. Krug, Adv. in Phys. 46 (1997) 139; Physica A 313 (2002) 47.

[3] P.G. de Gennes, Rev. Mod. Phys. 57 (1985) 827; I.E. Dzyaloshinskii, E.M. Lifshits, and L.P. Pitaevskii, Sov. Phys. - JETP 37 (1960) 161; J.N. Israelachvili, Intermolecular and Surface Forces (Academic, London, 1992).

[4] J. Espinosa, De-wetting of cobalt thin films on sapphire, Ph.D. Thesis, West Virginia University (2007), at http:/ / wvuscholar.wvu.edu.

[5] U. Thiele, J. Phys: Cond. Matt. 22 (2010) 084019.

[6] H.B.G. Casimir, Proc. K. Ned. Akad. 51 (1949), 79.

[7] E.M. Lifshitz, J. Exp. Theor. Phys. 29 (1955), 94.

[8] A. Bulgac and A. Wirzba, Phys. Rev. Lett. 87(2001), 120404.

[9] A. Wirzba, A. Bulgac and P. Magierski, J. Phys. A: Math. Gen. 39, 6815 (2006).

[10] A.J. Bray, Adv. Phys. 34 (1994) 357.

[11] M. Zinke-Allmang and L.C. Feldman, Appl. Surf. Sci. 52 (1991) 357.

[12] M.R. Krause et al. , J. Vac. Sci. Technol. A 24 (2006) 1480.

[13] K. B. Glaser and T. P. Witelski, Phys. Rev. E 67 (2003) 016302.

[14] F. Otto et al., SIAM J. Math. Anal. Vol. 38 (2006), 503. 


\section{Chapter 3}

\section{Simulations of interface dynamics and the cluster coarsening growth}

\subsection{Introduction}

In this Chapter, we describe the results of our interface dynamics simulations of the cluster coarsening dynamics. These simulations are the first step in our theoretical understanding of the effects of the long-range de-wetting interactions on the character of the cluster coarsening dynamics. As the next step in this direction, in the following chapters [Chs. 4 and 5], we will corroborate our simulations with an analytic theory of the cluster growth phenomena.

As detailed in this chapter, our simulations indicate that the long range de-wetting interactions produce significant early time departures from the asymptotic predictions based on the classical Young-Laplace cluster model, Sec. 2.5. Young-Laplace model gives the coarsening exponents that are correct only at asymptotically long times. The Young-Laplace asymptotic exponents are universal, that is, they do not depend on the details of the de-wetting interactions such as the value of $\omega$ in the potential in Eq. (4) of Ch. 2. In this chapter we will use our simulations results to elucidate the actual role played by the value of $\omega$. For this purpose, we will employ time dependent effective coarsening exponents. These effective coarsening exponents are powerful theoretical concepts that can be used to describe the cluster growth at any instant of the system evolution, during both late and early times. We will show that the effective coarsening 
exponents are very sensitive measures of the role played by the actual value of $\omega$ in the potential in Eq. (4) of Ch. 2. We find that smaller is the $\omega$, larger are the deviations of the effective exponents from their asymptotic values. These early time deviations are thus especially prominent for the small values of $\omega$, such as $\omega=2$ and $\omega=1$. We recall that these values of $\omega$ correspond to the experimentally significant de-wetting interactions, such as the van der Walls interaction $(\omega=2)$, and fermionic Casimir interaction $(\omega=1)$, discussed in Chapter 1. In fact, on the experimental side, a significant difference between the effective (early time) exponent and the asymptotic (late time) exponent for the growth of cluster height, has been indeed seen in the experiments, as noted in Ch. 1 [see Ref. [13] of our Ch. 1]. Our simulation results presented in this chapter, in combination with our analytic theory developed in following chapters [Chs. 4 and 5], explain these experimental findings in terms of long range de-wetting potentials.

To document the above anticipated findings, this chapter describes the results of our simulations of the interface dynamics of the rescaled model defined in Sec. 2.4. In this model, we will consider the de-wetting potentials with $\omega=1, \omega=2$, and $\omega=6$ in Eq. (4) of Ch. 2. In this and the following chapters, we focus on the physically interesting case with $D=2$; see Fig. 1 from our simulations. After an initial stage characterized by formation of pits, the interface eventually structures as an ensemble of clusters that grow via a coarsening process. By Fig. 1, the clusters are poly-disperse in sizes, with smaller than average clusters decaying by expelling their material into larger clusters [recall of Sec. 2.5 discussion]. Due to the decay of the small clusters, the total number of clusters $N(t)$ in the system decreases in time [see Fig. 2], while the average cluster size increases in time. This coarsening yields a steady growth of the average cluster height and cluster base radius, see Fig. 1. In the following section, we will introduce basic statistical quantities that can be used to characterize the evolving ensemble of growing clusters seen in Fig. 1 from our simulations. 


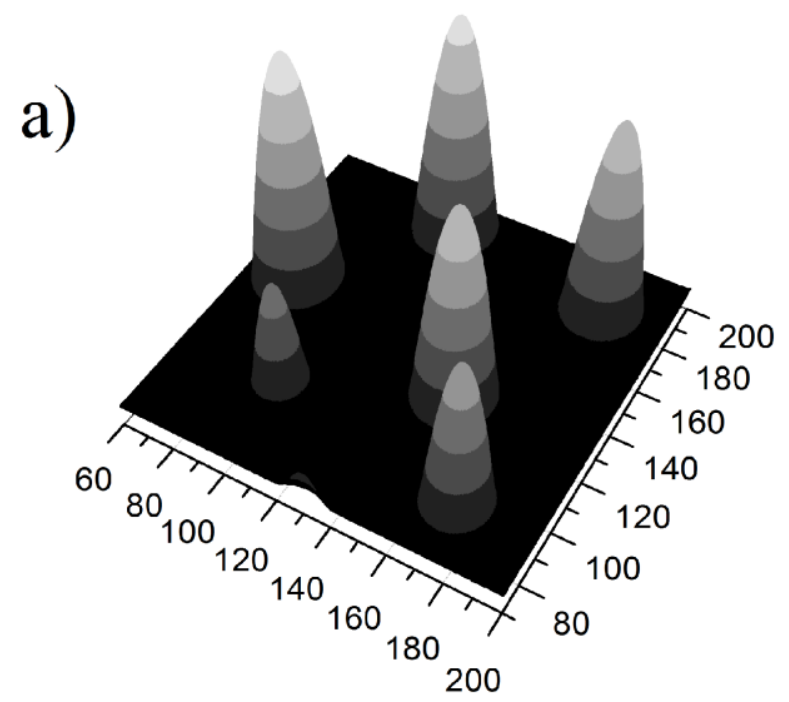

b)

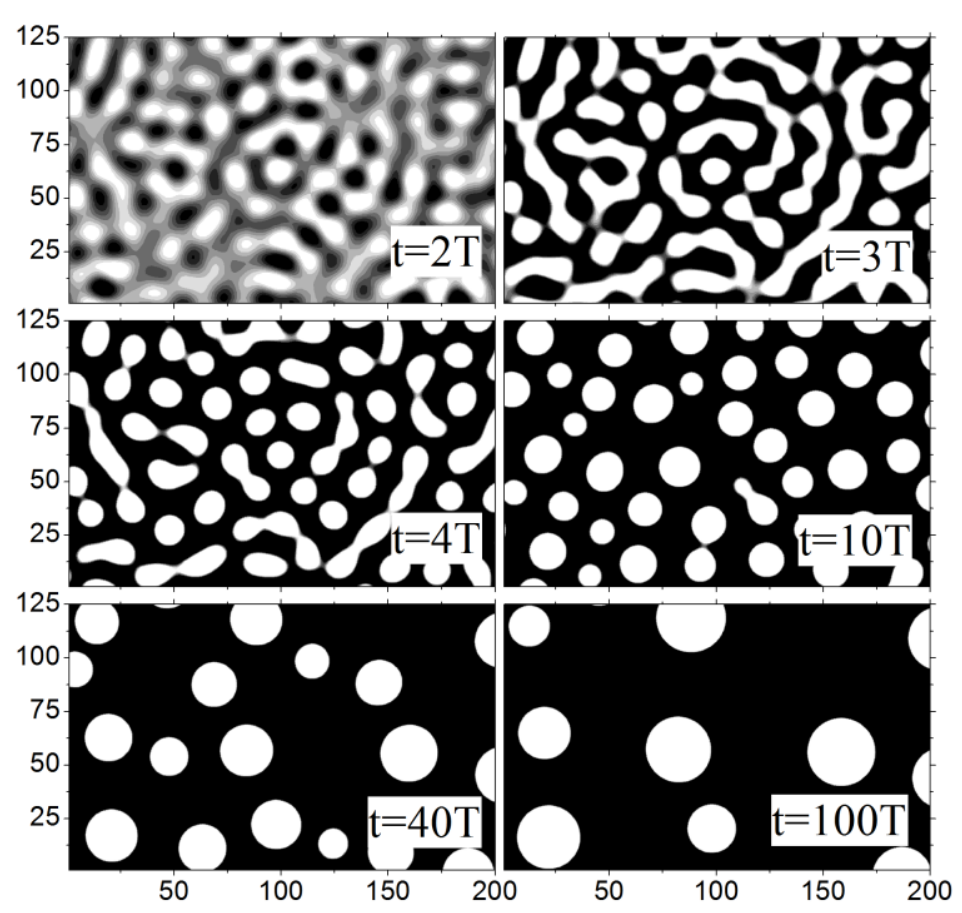

Fig. 1. Figure (a) exhibits the final stage of the $D=2$ thin film interface evolution, dominated by the presence of growing clusters. In figure (b), interface height contour plots showing film morphology evolution for $D=2$ and $\omega=1$, from the simulation of the rescaled model with dimensionless $t, \vec{x}, h$ (see Sec. 2.4). Here, $T$ is a time-scale conventionally set to correspond to the emergence of the transient worm-like morphology at $t \approx 3 T$. For $t<3 T$, the interface is in a time stage characterized by pit formation [as in Fig. 5(a) of Ch. 2]. For $t>3 T$, the interface is characterized by the formation of slowly growing clusters [as in figure (a) here]. 

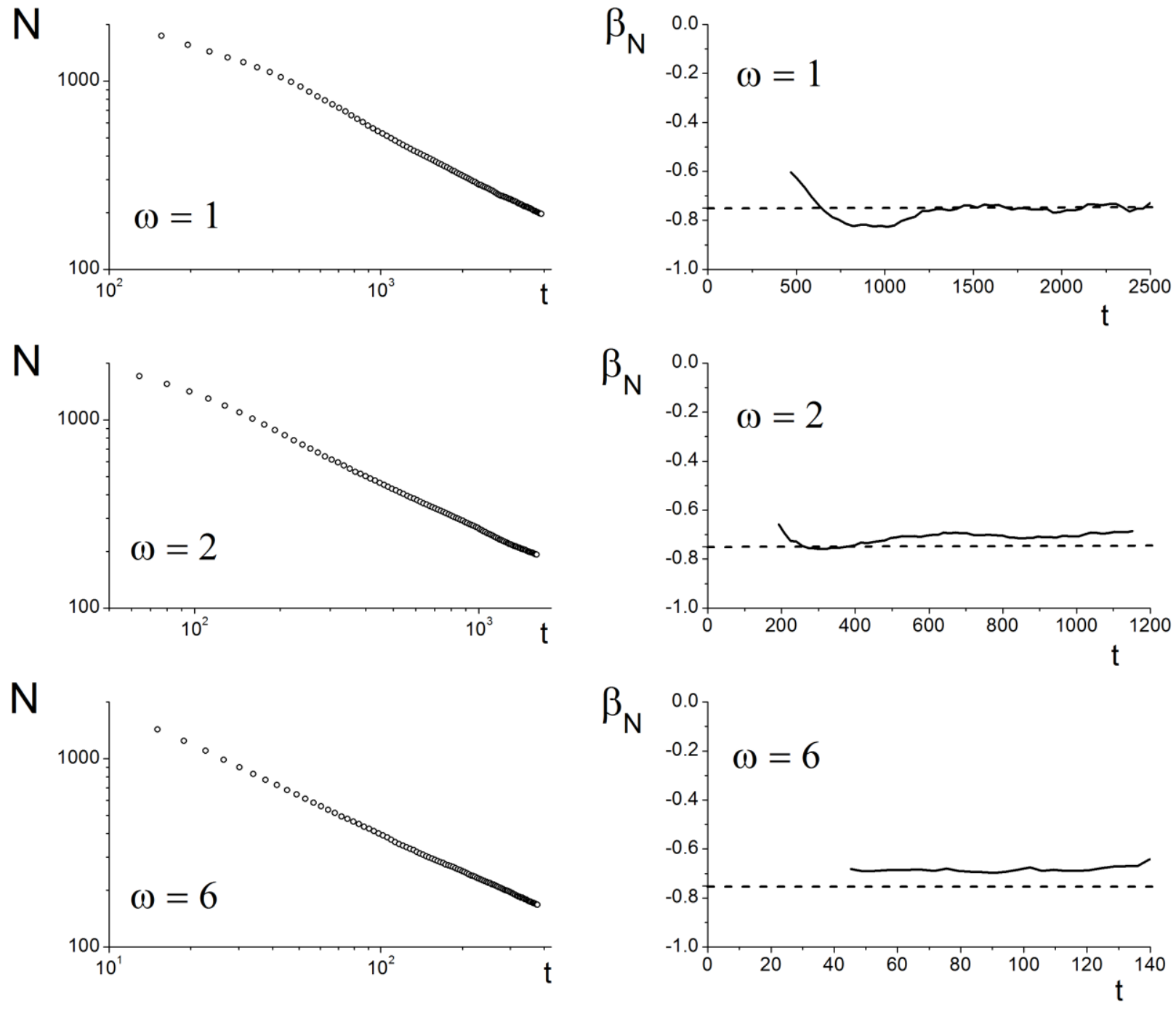

Fig. 2. Time evolution of the number of clusters $N(t)$ in the systems we simulated, with $\omega=1,2$, and 6 . The $N(t)$ displayed here is the average of the clusters numbers obtained from ten simulations done for each value of $\omega$. Also presented is the effective coarsening exponent, $\beta_{N}(t)=d \ln [N(t)] / d \ln (t)$. This exponent approaches the value of $-3 / 4$ at long times. There are however significant deviations away from this value, that are seen in the $\beta_{\mathrm{N}}(t)$ at early times. 


\subsection{Statistical quantities characterizing clusters and thin film interfaces}

In this section, we define a variety of statistical quantities we will use to quantify the evolving ensemble of clusters. In the following sections, these quantities will be extracted from our simulations. Thus, by using interface configurations such those seen in Fig. 1, we counted clusters to obtain their number $N(t)$ in the simulation sample at time $t$. We measured the heights of individual clusters, $H_{n}[n=1,2,3, \ldots, N(t)]$, to obtain the average cluster height,

$$
<H>=\frac{\sum_{n=1}^{N(t)} H_{n}}{N(t)} .
$$

We also measured the pressure at center of each cluster, $P_{n}$, to obtain the average cluster pressure,

$$
<P>=\frac{\sum_{n=1}^{N(t)} P_{n}}{N(t)} .
$$

Another important quantity characterizing our film is the size of the "wet" area, i.e., the area covered by clusters [the "white area" in Fig. 1]. To extract it, we used a convention involving a characteristic height scale $h^{*}$, below which the interface height rapidly (exponentially) approaches its asymptotic value $\approx h_{\min }$ [see Ch. 4 and Fig. 1(b) therein]. Thus, the interface sections with $h(\vec{x}, t)<h^{*}$ can be identified as "dry" area, whereas, the interface sections with $h(\vec{x}, t)>h^{*}$ can be identified as "wet" area, i.e., clusters. The total wet area is thus given by the surface integral, 


$$
A_{\text {wet }}(t)=\iint_{w e t} d^{2} \vec{x}=\iint_{\text {sample }} d^{2} \vec{x} \theta\left(h(\vec{x}, t)-h^{*}\right) .
$$

Here, $\theta(z)$ is the usual step function $[\theta(z)=1$ for $z>0$, and $\theta(z)=0$ for $z<0]$. The total wet area Eq. (3) is obviously smaller than the entire substrate area,

$$
A_{\text {sample }}=\iint_{\text {sample }} d^{2} \vec{x} .
$$

The area fraction occupied by clusters ("wet area fraction") is thus,

$$
\phi(t)=\frac{A_{\text {wet }}(t)}{A_{\text {sample }}}=\frac{\iint_{\text {wet }} d^{2} \vec{x}}{\iint_{\text {sample }} d^{2} \vec{x}}=\frac{\iint_{\text {sample }} d^{2} \vec{x} \theta\left(h(\vec{x}, t)-h^{*}\right)}{\iint_{\text {sample }} d^{2} \vec{x}} .
$$

We stress that the above employed height scale $h^{*}$ is not a sharply defined quantity. One possible choice would be to identify $h^{*}$ with the inflection point of the de-wetting potential $\Phi(h)$, i.e. $\Phi^{\prime \prime}\left(h^{*}\right)=0$. We have however decided for a different convention defining the $h^{*}$ via the condition that the third derivative $\Phi(h)$ vanishes at $h^{*}$. For the here interesting rescaled model potential in Eqs. (32) and (33) of Ch. 2, this condition gives $h^{*}=1$ for any $\omega$ [as discussed in Sec. 2.4].

The base areas of the clusters are non-overlapping [see Fig. 1], so the total wet area is simply the sum of all clusters base areas,

$$
A_{w e t}(t)=\sum_{n=1}^{N(t)} A_{n} .
$$

Thus, by Eqs. (6) and (3), the average cluster base area is

$$
<A>=\frac{\sum_{n=1}^{N(t)} A_{n}}{N(t)}=\frac{\iint_{\text {sample }} d^{2} \vec{x} \theta\left(h(\vec{x}, t)-h^{*}\right)}{N(t)} .
$$


We also measured the base area of each individual cluster, $A_{n}[n=1,2,3, \ldots, N(t)]$. Thus cluster bases are nearly circular [see Fig. 1 from our simulations], so one can introduce the base radius of the $\mathrm{n}$-th cluster via

$$
\pi R_{n}^{2}(t)=A_{n} \Rightarrow R_{n}=\sqrt{\frac{A_{n}}{\pi}} .
$$

Using (8), we calculated the average cluster base radius via,

$$
<R>=\frac{\sum_{n=1}^{N(t)} R_{n}}{N(t)} .
$$

Another quantity of interest is the total volume of the film inside clusters, i.e., the volume of the film covering wet areas with $h(\vec{x}, t)>h^{*}$. Recall that the actual film thickness is

$$
\tilde{h}(\vec{x}, t)=h(\vec{x}, t)-h_{\min } ;
$$

see Sec. 2.3. Thus, for example, the n-th cluster height $H_{n}$ is given by Eq. (10) with $\vec{x}$ being the center of cluster base area. The total volume of the film covering wet areas $\left[h(\vec{x}, t)>h^{*}\right]$ is

$$
\iint_{w e t} d^{2} \vec{x} \tilde{h}(\vec{x}, t)=\iint_{\text {sample }} d^{2} \vec{x} \theta\left(h(\vec{x}, t)-h^{*}\right) \cdot\left[h(\vec{x}, t)-h_{\min }\right] .
$$

This volume is the sum of volumes of individual clusters, $V_{n}[n=1,2,3, \ldots, N(t)]$, so,

$$
\sum_{n=1}^{N(t)} V_{n}=\iint_{\text {sample }} d^{2} \vec{x} \theta\left(h(\vec{x}, t)-h^{*}\right) \cdot\left[h(\vec{x}, t)-h_{\min }\right] .
$$

Thus, the average volume of a cluster is given by

$$
<V>=\frac{\sum_{n=1}^{N(t)} V_{n}}{N(t)}=\frac{\iint_{\text {sample }} d^{2} \vec{x} \theta\left(h(\vec{x}, t)-h^{*}\right) \cdot\left[h(\vec{x}, t)-h_{\min }\right]}{N(t)} .
$$


It will be convenient to introduce the concept of spatial average, defined via

$$
<f(\vec{x}, t)\rangle_{s p}=\frac{\iint_{\text {sample }} d^{2} \vec{x} f(\vec{x}, t)}{\iint_{\text {sample }} d^{2} \vec{x}},
$$

for any function $f(\vec{x}, t)$. Thus, the wet area fraction Eq. (5) can be expressed briefly as,

$$
\phi(t)=<\theta\left(h(\vec{x}, t)-h^{*}\right)>_{s p} .
$$

Also interesting are the spatial averages in the form of the moments of the local film thickness,

$$
M_{n}(t)=<[\tilde{h}(\vec{x}, t)]^{n}>_{s p}=<\left(h(\vec{x}, t)-h_{\min }\right)^{n}>_{s p}=\frac{\iint_{\text {sample }} d^{2} \vec{x}\left(h(\vec{x}, t)-h_{\min }\right)^{n}}{\iint_{\text {sample }} d^{2} \vec{x}} .
$$

For $n=1$, the above reduces to

$$
M_{1}=<\tilde{h}(\vec{x}, t)>=\frac{\iint_{\text {sample }} d^{2} \vec{x}\left(h(\vec{x}, t)-h_{\min }\right)}{\iint_{\text {sample }} d^{2} \vec{x}}=\frac{V_{\text {film }}}{A_{\text {sample }}} .
$$

By Ch. 2 discussions, total film volume $V_{\text {film }}$ is conserved. Thus, the first order moment $M_{1}$ in Eq. (17) does not depend on time. However, other moments $\left(M_{2}, M_{3}, \ldots\right)$ do depend on time. Note that, with our initial condition [discussed in Sec. (2.4)], the moment $M_{1}$ is simply equal to the initial film thickness,

$$
M_{1}=\tilde{h}_{0}=h_{0}-h_{\min } .
$$

An interesting statistical quantity of the thin film is the restricted average height, $h_{\text {rest }}$ defined as the spatial average of the local film thickness done however only over the wet area occupied by clusters, that is, 


$$
h_{\text {rest }}(t)=\frac{\iint_{w e t} d^{2} \vec{x} \tilde{h}(\vec{x}, t)}{\iint_{w e t} d^{2} \vec{x}}=\frac{\iint_{\text {sample }} d^{2} \vec{x} \theta\left(h(\vec{x}, t)-h^{*}\right)\left[h(\vec{x}, t)-h_{\min }\right]}{\iint_{\text {sample }} d^{2} \vec{x} \theta\left(h(\vec{x}, t)-h^{*}\right)} .
$$

By Eqs. (7), (13), and (19), we see that,

$$
h_{\text {rest }}(t)=\frac{<V>}{<A>} .
$$

Finally, also interesting are nonlocal surface averages, such the height-height correlation function,

$$
K(\vec{r}, t)=<\tilde{h}(\vec{x}, t) \cdot \delta \tilde{h}(\vec{x}+\vec{r}, t)>_{s p}=\frac{\iint_{\text {sample }} d \vec{x} \delta \tilde{h}(\vec{x}, t) \cdot \delta \tilde{h}(\vec{x}+\vec{r}, t)}{\iint_{\text {sample }} d \vec{x}}
$$

with $\delta \tilde{h}(x, t)=\tilde{h}(x, t)-<\tilde{h}(x, t)>$.

In the following sections of this chapter, we will discuss the dynamics of the numerous statistical quantities introduced in this section, by using the results obtained from our simulations for three different values of $\omega[\omega=1,2$, and 6].To suppress statistical fluctuations, all statistical quantities are obtained by doing average of data obtained from 10 statistically independent simulations done for each studied value of $\omega$.

\subsection{Dynamics of cluster sizes and pressure}

In the Figs. 3 through 6, we display our simulations results for the time evolution of the four basic statistical quantities characterizing cluster ensemble: average cluster volume $\langle V\rangle$, average cluster pressure $\langle P\rangle$, average cluster height $\langle H\rangle$, and cluster radius $R$. These quantities are extracted from our simulations results, using their definitions in Eqs. (1), (2), (9), and (13). 

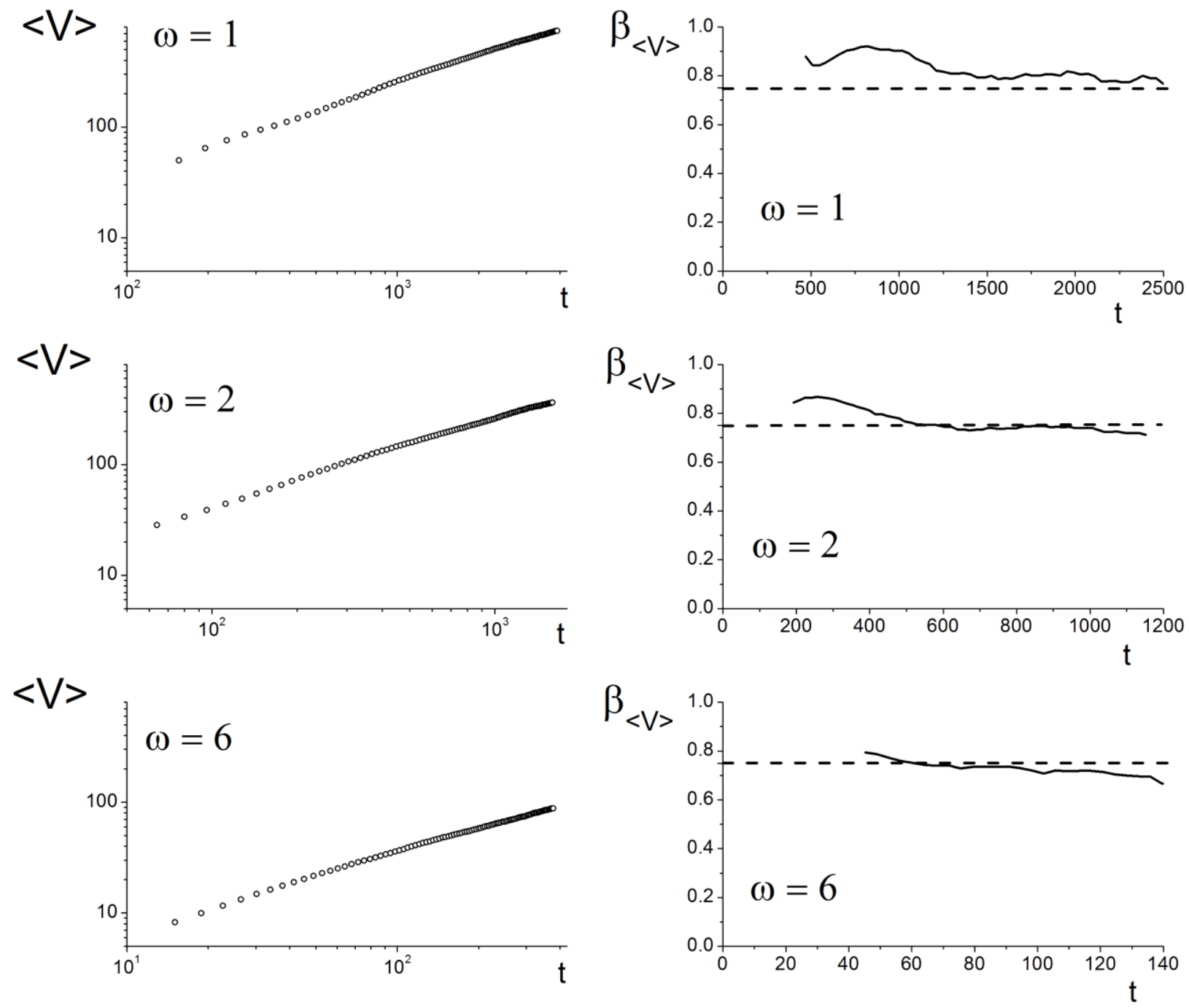

Fig. 3. Temporal evolution of the clusters average volume $\langle V\rangle$ in the systems we simulated, with $\omega=1,2$, and 6 . We also present the evolution of the corresponding effective coarsening exponent, $\beta_{<V>}(t)=d \ln [<V>V / d \ln (t)$. This exponent approaches the value of $+3 / 4$ at long times. There are however significant deviations away from this value, that are seen in the $\beta_{<V_{>}}(t)$ at early times. 

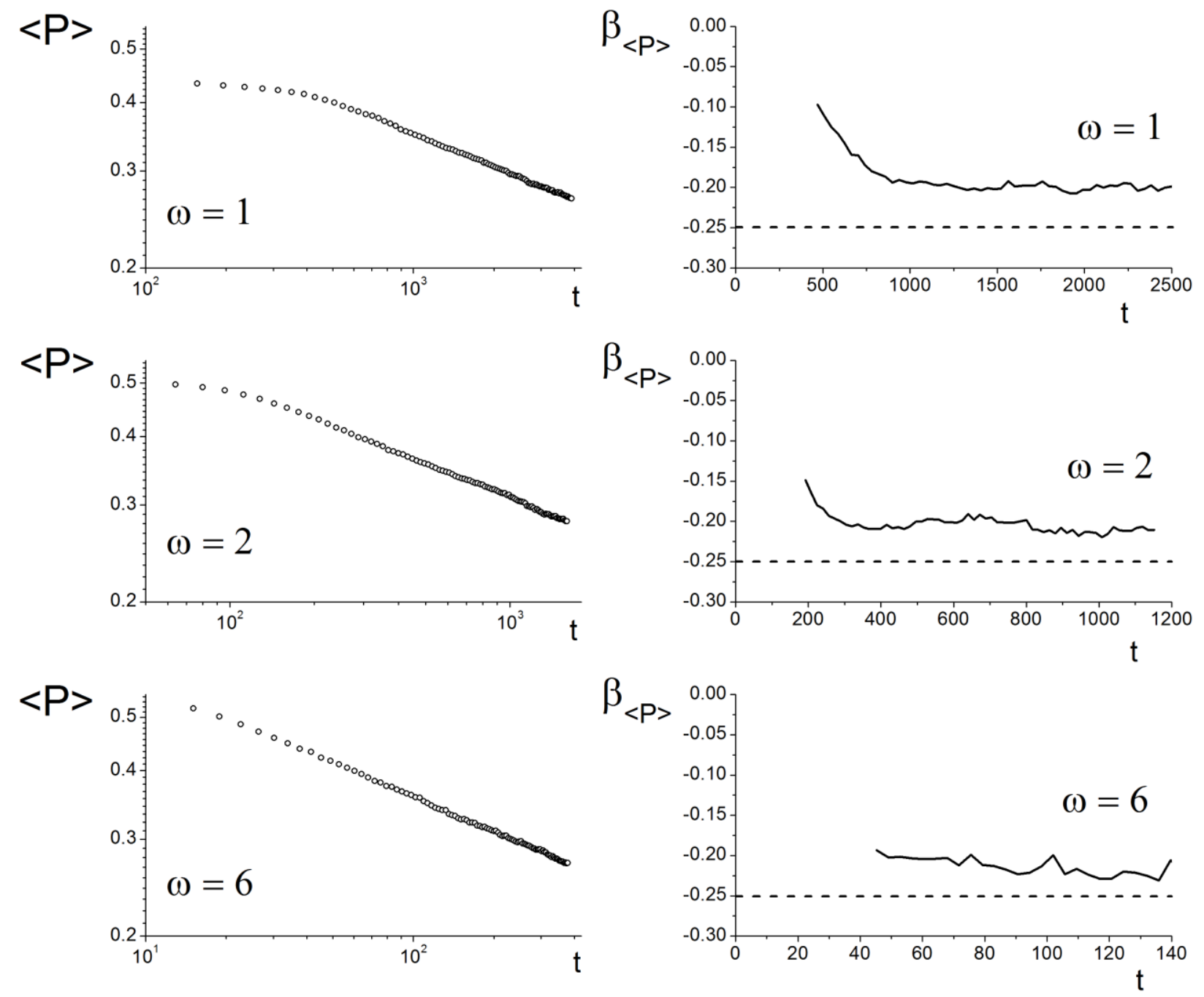

Fig. 4. Temporal evolution of the clusters average pressure $\langle P\rangle$ in the systems we simulated, with $\omega=1,2$, and 6 . We also present the evolution of the corresponding effective coarsening exponent, $\beta_{<P>}(t)=d \ln [<P>V / d \ln (t)$. This exponent approaches the value of $-1 / 4$ at long times. There are however significant deviations away from this value, that are seen in the $\beta_{<\mathrm{P}\rangle}(t)$ at early times. 

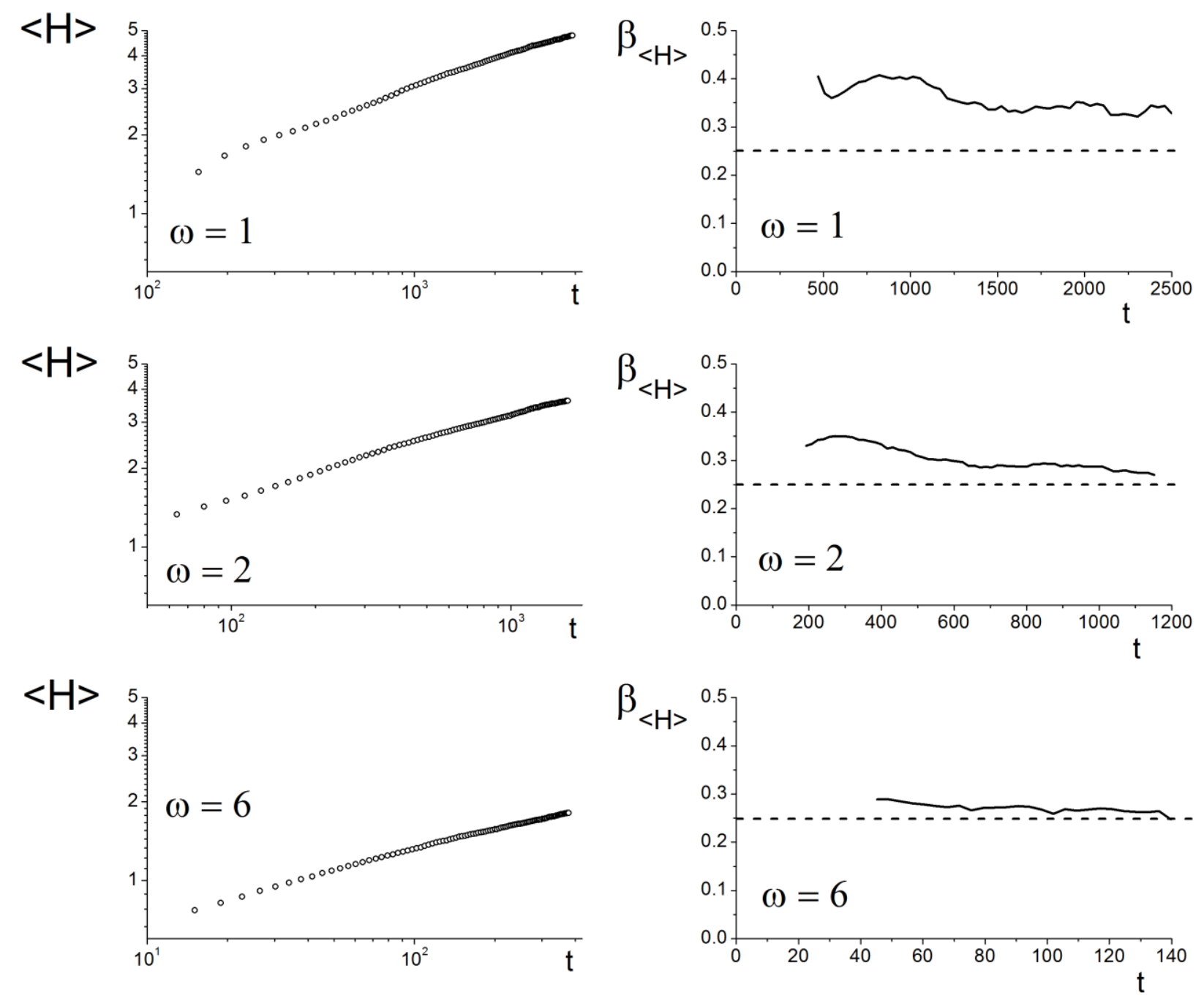

Fig. 5. Time evolution of the clusters average height $\langle H>$ in the systems we simulated, with $\omega=1,2$, and 6. We also present the evolution of the corresponding effective exponent, $\beta_{<H>}(t)=d \ln [<H>V / d \ln (t)$. This exponent approaches the value of $+1 / 4$ at long times. There are however significant deviations away from this value, that are seen in the $\beta_{<\mathrm{H}>}(t)$ at early times. 


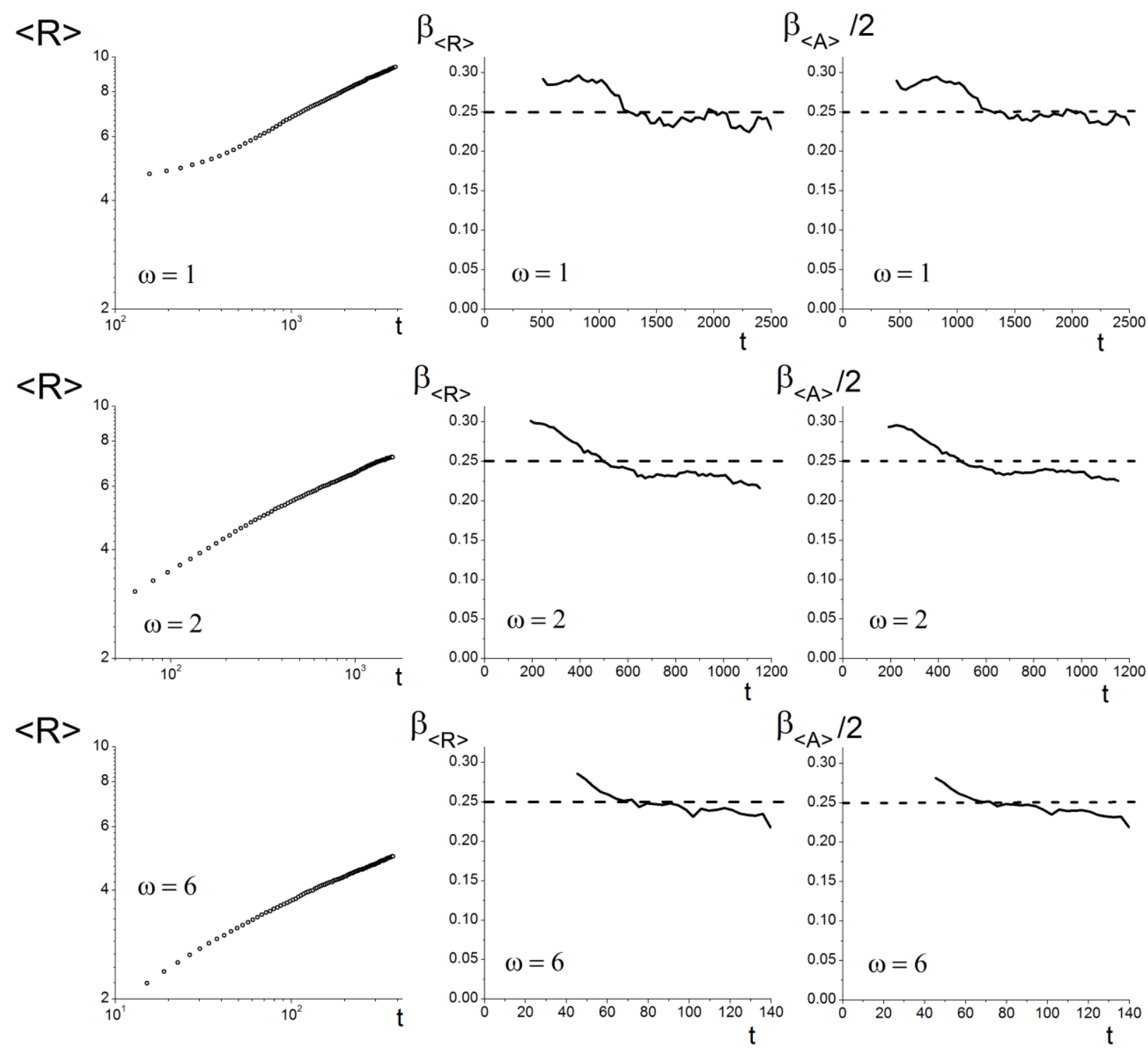

Fig. 6. Time evolution of the clusters average base radius $\langle R>$ in the systems we simulated, with $\omega=1,2$, and 6 . We also present the evolution of the corresponding effective exponent, $\beta_{\mathrm{R}}(t)=$ $d \ln [<R>V / d \ln (t)$. This exponent approaches the value of $+1 / 4$ at long times. There are however significant deviations away from this value, that are seen in the $\beta_{<\mathrm{R}>}(t)$ at early times. For comparison, we also display $\beta_{<A>}(t) / 2$, where $\beta_{<A>}(t)=d \ln [<A>V / d \ln (t)$ is the effective exponent for the growth of cluster base area. 
In the Figs. 3 through 6, we also display the evolution of the effective coarsening exponents, defined by,

$$
\beta_{<V>}(t)=\frac{d \ln (<V>)}{d \ln (t)}, \quad \beta_{<P>}(t)=\frac{d \ln (<P>)}{d \ln (t)}, \quad \beta_{<H>}(t)=\frac{d \ln (<H>)}{d \ln (t)}, \quad \beta_{<R>}(t)=\frac{d \ln (<R>)}{d \ln (t)} .
$$

From the Figs. 3 through 6, we see that these effective exponents depend on time $t$. The exponents seen in these figures deviate from the asymptotic values expected at long times, on the basis of the Young-Laplace model for clusters [Sec. 2.5]. By the Sec. 2.5 results (for $D=2$ ), these asymptotic exponents are given by,

$$
\beta_{<H>}(t=\infty)=\beta_{<R>}(t=\infty)=\frac{1}{4},
$$

and, by Eq. (49) of Ch.2,

$$
\beta_{<V>}(t=\infty)=\beta_{<H>}(t=\infty)+2 \beta_{<R>}(t=\infty)=\frac{3}{4},
$$

while, by Eq. (44) of Ch.2,

$$
\beta_{<P>}(t=\infty)=-\beta_{<H>}(t=\infty)=-\frac{1}{4} .
$$

The above asymptotic (Young-Laplace) coarsening exponents are universal, i.e., they do not depend on the de-wetting potential details such as the value of $\omega$ in Eq. (4) of Ch. 2. However, the simulations results displayed in the Figs. 3 through 6 do evidence significant early time departures away from the above universal long time coarsening exponents. Moreover, the simulations results indicate that these departures are correlated with the long range character of the potential. For example, from Fig. 5, for a large $\omega=6$ (essentially, a short range de-wetting potential), the early time range cluster height exponent $\beta_{<\mathrm{H}>}$ is about 0.28 , so it is close to the universal asymptotic $\beta_{<\mathrm{H}>}=0.25$. On the other hand, for genuine long-range potentials with for $\omega=1$ and $\omega=2$, the early 
time range exponent $\beta_{<H}>$ is about 0.33 , i.e., it deviates significantly from the asymptotic $\beta_{<\mathrm{H}>}=0.25$. It is of direct physical interest to understand these effects. Indeed, the potentials $\omega=1$ and $\omega=2$ are physically interesting since they corresponding to van der Waals interaction $(\omega=2)$, and to fermionic Casimir interaction $(\omega=1)$; see Chapters 1 and 2 discussions. In addition, on the experimental side, an effective (early time) exponent for the growth of cluster height, estimated to be $\beta_{H}^{\text {eff }} \approx 0.33$, has been indeed reported for Sn clusters on silicon, see Ref. [13] of Ch. 1. In view of the above discussion, by the results of our simulations in figure 5, we argue that the experimentally seen coarsening exponent emerges as an effect of the long-range de-wetting interactions.

The above simulation results suggest that the deviations from the Young-Laplace model are larger for smaller values of $\omega$, i.e., for longer ranged de-wetting interactions. To illustrate this point, in Fig. 7 we plot the quantity

$$
\frac{\frac{2<H>}{<R>}}{m_{Y}} .
$$

By the Eq. (46) of Ch. 2, we have for the n-th cluster (in the Young-Laplace limit)

$$
2 H_{n}=m_{Y} R_{n} \Rightarrow 2<H>=m_{Y}<R>\text {. }
$$

Thus the quantity in Eq. (25) is exactly 1 in the Young-Laplace limit. However, from our simulation results in Fig. 7 (lower panel) we see that the aspect ratio $2<H>\mid<R>$ is, at early times, significantly smaller than the Young angle slope $m_{Y}$, especially for the smaller values of $\omega$, i.e., for longer range de-wetting interactions. As documented in Fig. 7(upper panel), this effect is correlated with the magnitude of the departures of the effective coarsening exponents from their asymptotic values, which are larger for smaller values of $\omega$, i.e., for the longer range interactions.

This breakdown of the classical Young-Laplace model effect will be discussed analytically in the next chapter [Ch. 4] as well as in Chapter 5 in which we develop a 
novel cluster dynamics theory capable to analytically explain the results of this chapter simulations as well as the related results from the experiments such as those of Ref. [13] of Ch. 1.
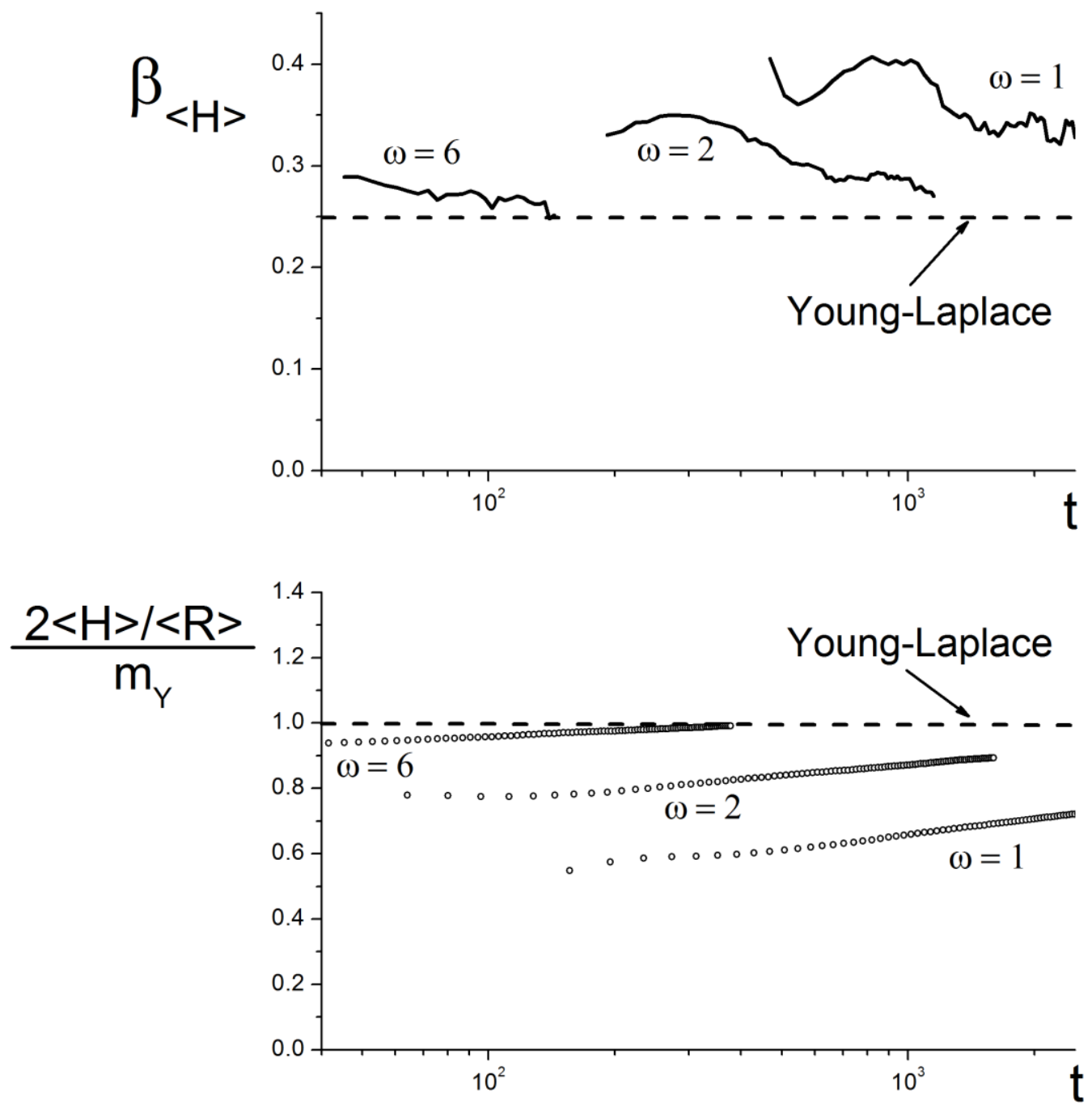

Fig. 7: The figure qualitatively documents the correlation between the failure of the Young-Laplace model prediction for cluster aspect ratio (lower panel), with the magnitude of the deviations of the effective exponent $\beta_{H}$ away from its asymptotic value (upper panel). Both effects increase with decreasing $\omega$. 
Finally, also interesting is the scaling of the average cluster base area Eq. (7). The associate effective coarsening exponent is,

$$
\beta_{<A>}(t)=\frac{d \ln (<A>)}{d \ln (t)} .
$$

Crudely, $<A>$ is approximately $\pi<R>2$, so one may expect,

$$
\beta_{<A>}(t) \approx 2 \beta_{<R>}(t) \Rightarrow \frac{\beta_{<A>}(t)}{2}=\beta_{<R>}(t)
$$

at any $t$, and, in particular,

$$
\beta_{<A>}(t=\infty)=2 \beta_{<R>}(t=\infty)=\frac{1}{2},
$$

in the infinite time, Young-Laplace limit. To check the validity of the approximate relation (28), in Fig. 6, we plot our simulation results for both $\beta<A>/ 2$ and $\beta<R>$. The approximate character of Eq. (28) is evident from these plots.

\subsection{Other statistical properties of the thin film interface}

In Fig. 8, we display the time evolution of the area fraction occupied by clusters, i.e., the wet area fraction $\phi$ defined in Eq. (5). In the figure, we also plot the corresponding effective coarsening exponent,

$$
\beta_{\phi}(t)=\frac{d \ln (\phi)}{d \ln (t)} .
$$

This exponent is expressible in terms of the exponents for cluster base area Eq. (27) and cluster number $N(t)$, 

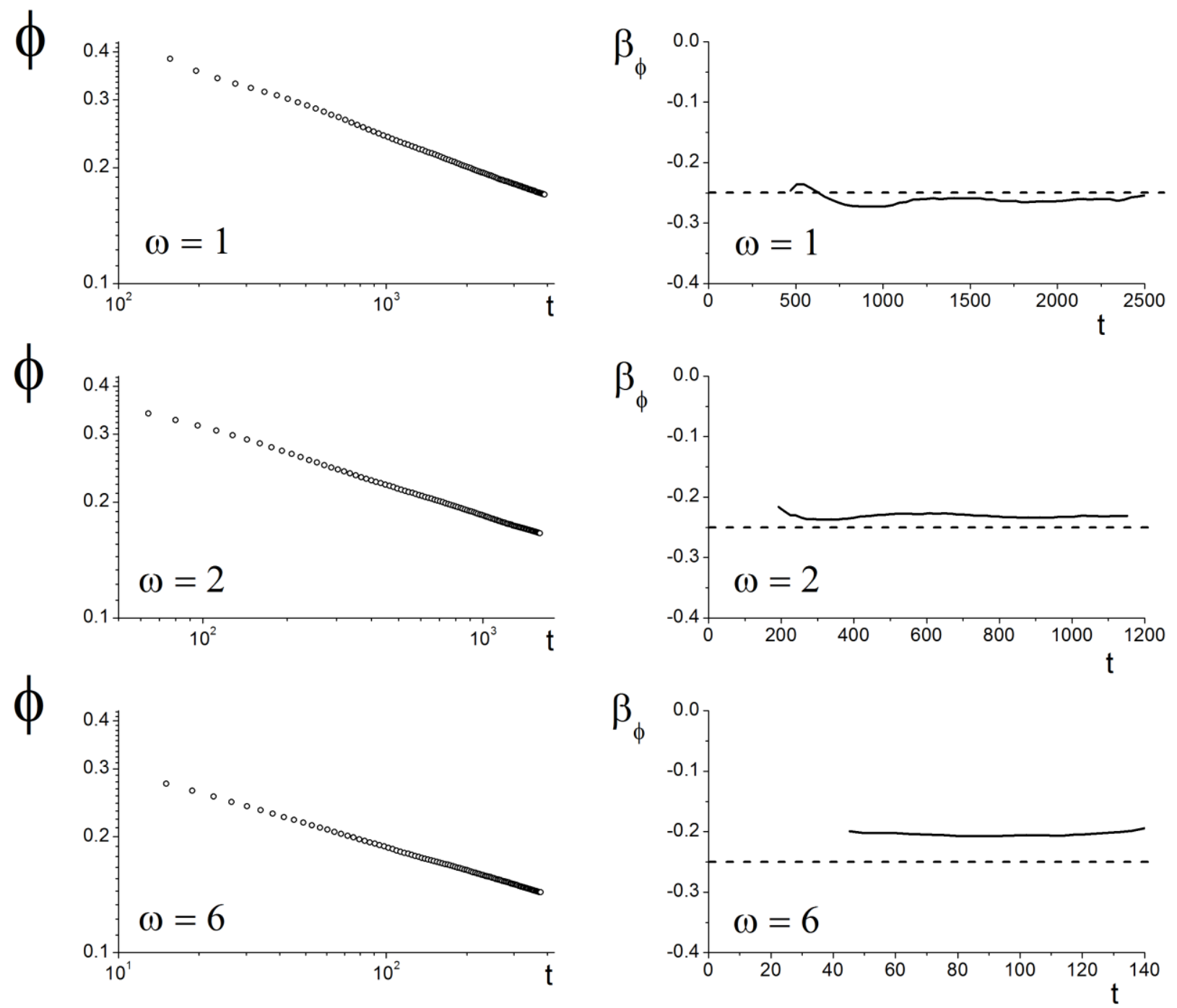

Fig. 8. The time evolution of the area fraction occupied by clusters, i.e., the wet area fraction $\phi$ in the systems we simulated, with $\omega=1,2$, and 6 . In the figure, we also plot the corresponding effective coarsening exponent $\beta_{\phi}$. It approaches the value of $-1 / 4$ at long times. 


$$
\beta_{N}(t)=\frac{d \ln [N(t)]}{d \ln (t)},
$$

displayed earlier in Fig. 2. Indeed, by the Eqs. (5) and (7),

$$
\phi(t)=\frac{N(t)<A>}{A_{\text {sample }}} .
$$

Thus,

$$
\beta_{\phi}(t)=\beta_{N}(t)+\beta_{<A>}(t) .
$$

As much as for the previously discussed exponents, in Fig. 8 we see significant $\omega$-dependent deviations of $\beta_{\phi}(t)$ away from its universal asymptotic value,

$$
\beta_{\phi}(t=\infty)=\beta_{N}(t=\infty)+\beta_{<A>}(t=\infty)=-\frac{1}{4} .
$$

Above, we used the Eq. (29) and the result

$$
\beta_{N}(t=\infty)=-\frac{3}{4},
$$

discussed in the following.

In Fig. 9, we display the time evolution of the average restricted height ; see Eqs. (19) and $\left(19^{\prime}\right)$. In the figure, we also plot the corresponding effective coarsening exponent,

$$
\beta_{h_{\text {rest }}}(t)=\frac{d \ln \left(h_{\text {rest }}\right)}{d \ln (t)}
$$

By Eq. $\left(19^{\prime}\right)$, this exponent is expressible in terms of the exponents for cluster base area Eq. (27) and cluster number volume [see Eq. (21)], 

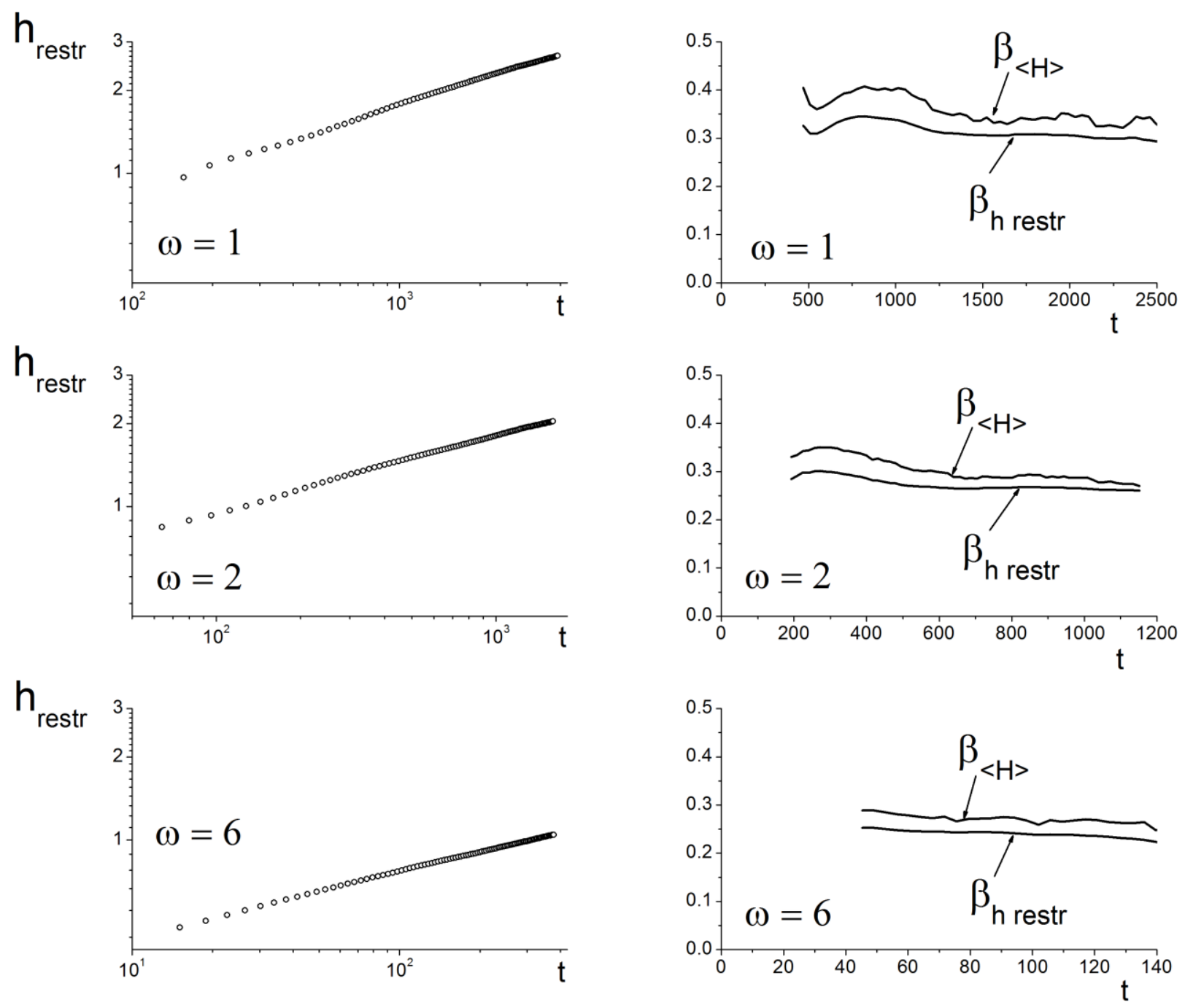

Fig. 9. The time evolution of the restricted average height $h_{\text {rest }}$ in the systems we simulated, with $\omega=1$, 2, and 6. In the figure, we also plot the corresponding effective coarsening exponent. It approaches the value of $1 / 4$ at long times. 


$$
\beta_{h_{\text {rest }}}(t)=\beta_{<V>}(t)-\beta_{<A>}(t) .
$$

As much as for the previously discussed exponents, in Fig. 8 we see significant deviations of this exponent away from its asymptotic value,

$$
\beta_{h_{\text {rest }}}(t=\infty)=\beta_{<V>}(t=\infty)-\beta_{<A>}(t=\infty)=\frac{1}{4} .
$$

Figure 10 from our simulations documents an interesting approximate relation between the effective exponents $\beta_{\mathrm{N}}(\mathrm{t})$ and $\beta_{<\mathrm{v}>}(\mathrm{t})$, of the form,

$$
-\beta_{N}(t) \approx \beta_{<V>}(t) .
$$

The origin of the relation Eq. (39) is in the fact that the (conserved) film volume $V_{\text {film }}$ is almost entirely accumulated inside the clusters, i.e., over the wet area fraction. In terms of Eq. (13), this means,

$$
\begin{aligned}
& N(t)<V>=\sum_{n=1}^{N(t)} V_{n}=\iint_{\text {sample }} d^{2} \vec{x} \theta\left(h(\vec{x}, t)-h^{*}\right) \cdot\left[h(\vec{x}, t)-h_{\min }\right] \\
& \approx \iint_{\text {sample }} d^{2} \vec{x}\left[h(\vec{x}, t)-h_{\min }\right]=V_{\text {film }}
\end{aligned}
$$

Thus, we have the relation

$$
N(t)<V>\approx V_{\text {film }}=\text { const. , }
$$

implying the exponent relation (39). From the simulations result in Fig. 10, we indeed see the approximate character of the relation (39). From the figure, we also see that the difference between $-\beta_{N}(t)$ and $\beta<v>(t)$ decreases with time. Thus, asymptotically,

$$
\beta_{N}(t=\infty)=-\beta_{<V>}(t=\infty)=-\frac{3}{4},
$$



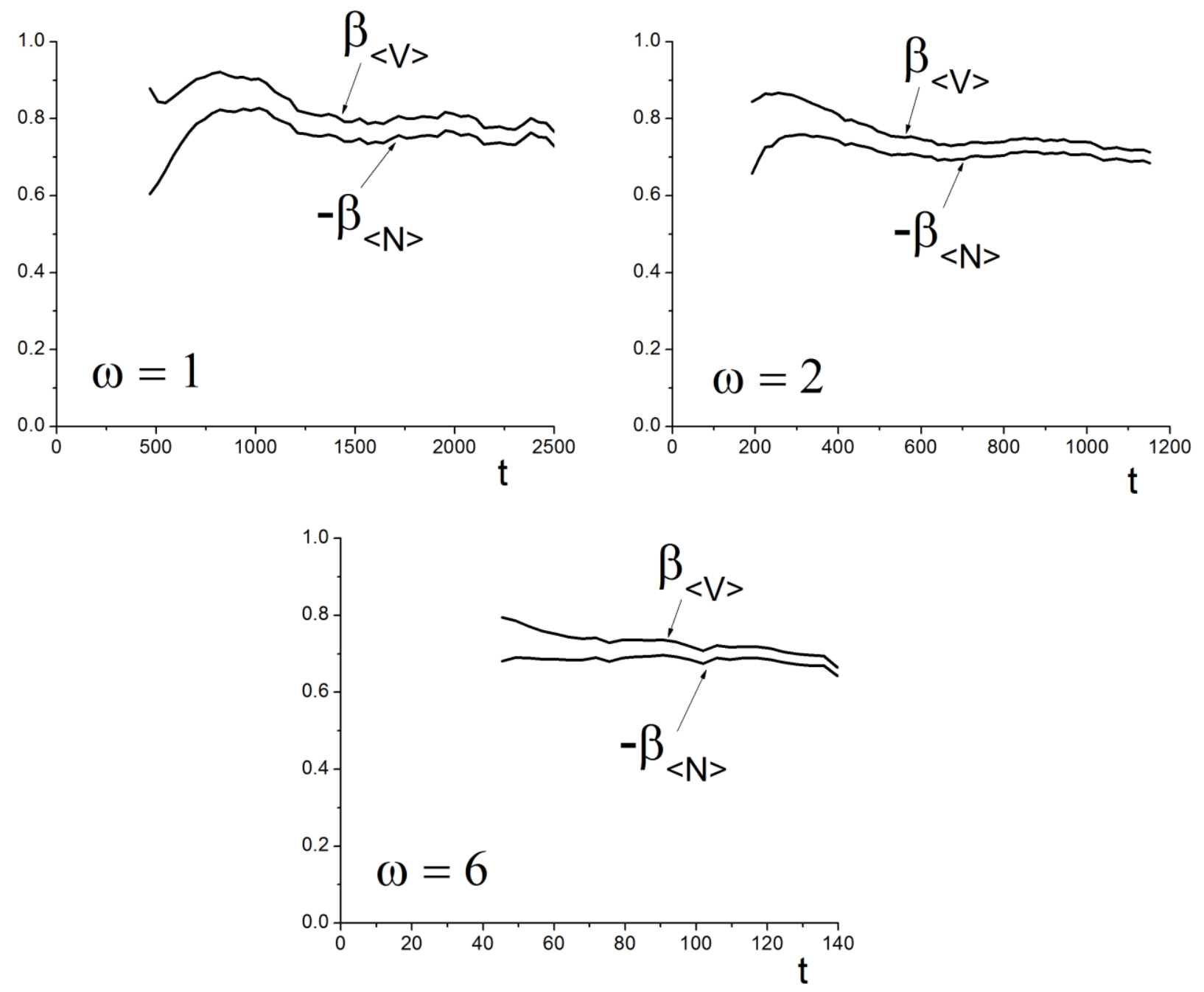

Fig. 10. Simulations results documenting that $-\beta_{N}(t)$ is approximately equal to $\beta_{<V>}(t)$. 
as anticipated before in the Eq. (35).

Yet another interesting approximate exponents relation is seen in Fig. 11 from our simulations. Therein we plot the growth of the second moment of the local film thickness, defined by the Eq. (16) with $n=2$. In the Fig. 11, we plot the effective coarsening exponent going with this quantity growth,

$$
\beta_{<\tilde{h}^{2}>}(t)=\frac{d \ln \left(<\tilde{h}^{2}>\right)}{d \ln (t)},
$$

and, in the same figure, we compare this exponent with the exponent $\beta<H>$ for the growth of the cluster average height. We see that the relation,

$$
\beta_{<\tilde{h}^{2}>}(t) \approx \beta_{<H>}(t)
$$

is suggested by the plots in Fig. 11.

Finally, in the Figs. 12 and 13, we display our results obtained by calculating the height-height correlation function $K(r, t)$, Eq. (20). The figure 12 displays this correlation function obtained at several instants of evolution time. The most prominent feature of the $K(r, t)$ is its central peak originating from cluster spatial self-correlations (of a cluster with itself). The width $w(t)$ of the self-correlation peak (indicated in Fig. 12), is, approximately,

$$
w(t) \approx 2<R>.
$$

Thus, we expect that the effective coarsening exponent of $w(t)$ is nearly the same as that of $<R>$. This is indeed the case; see Fig. 13 and compare it with Fig. 6. 

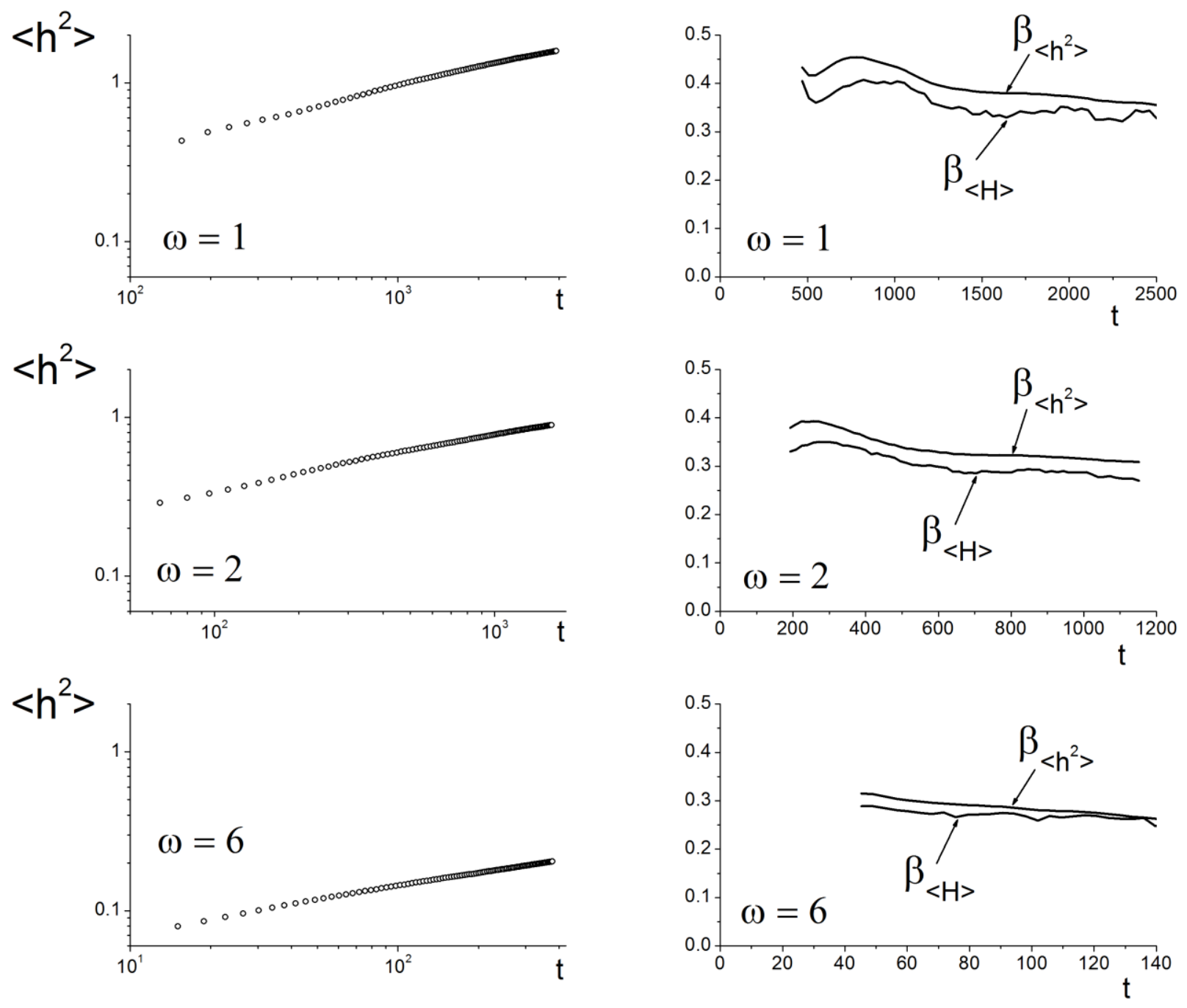

Fig. 11. Simulations results documenting the Eq. (44) 

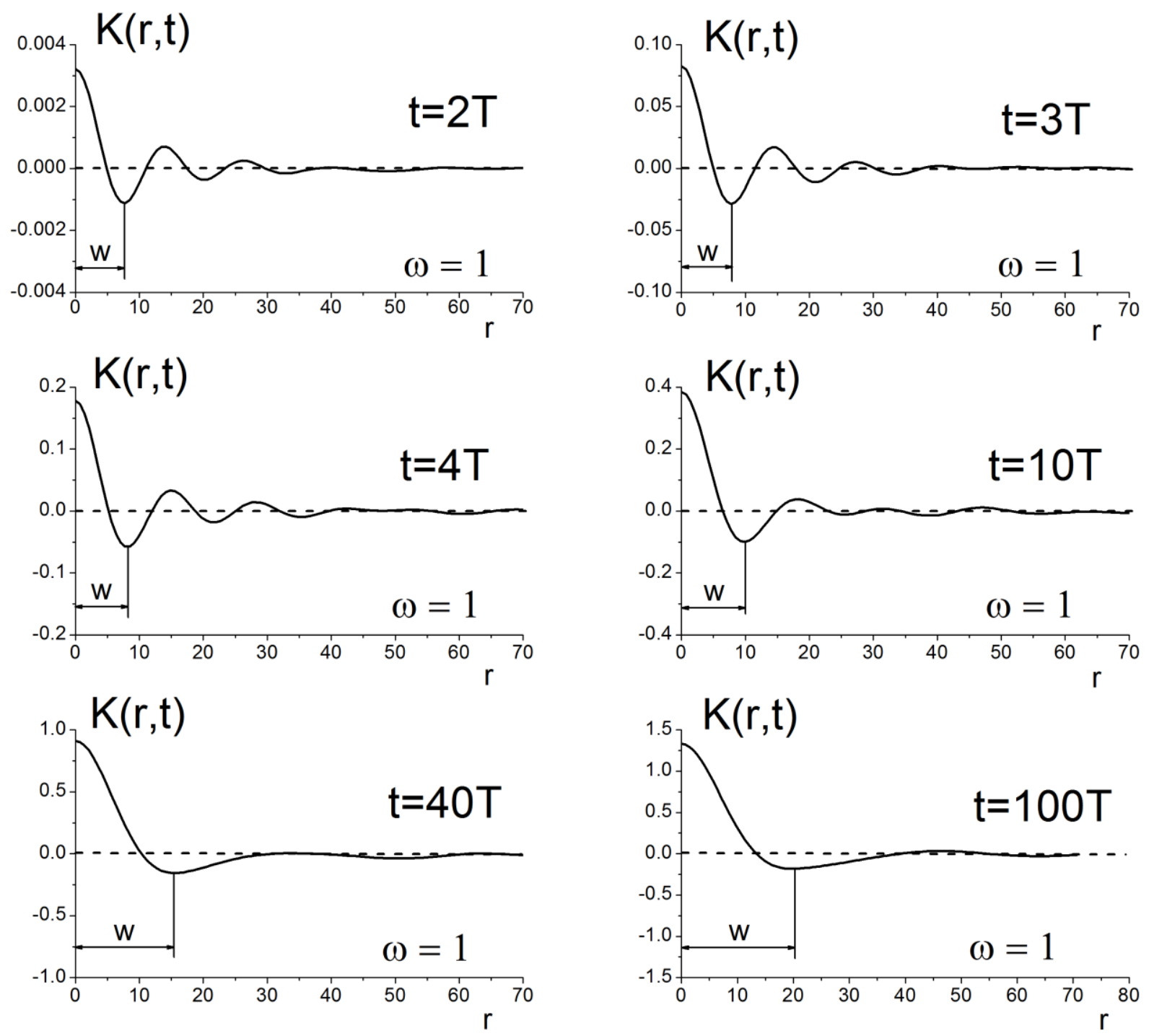

Fig. 12. The height-height correlation function $K(r, t)$ obtained at several instants of evolution time.

The most prominent feature of the $K(r, t)$ is its central peak originating from single cluster spatial selfcorrelations. The width $w(t)$ of the self-correlation peak is approximately equal to $2<R>$. 

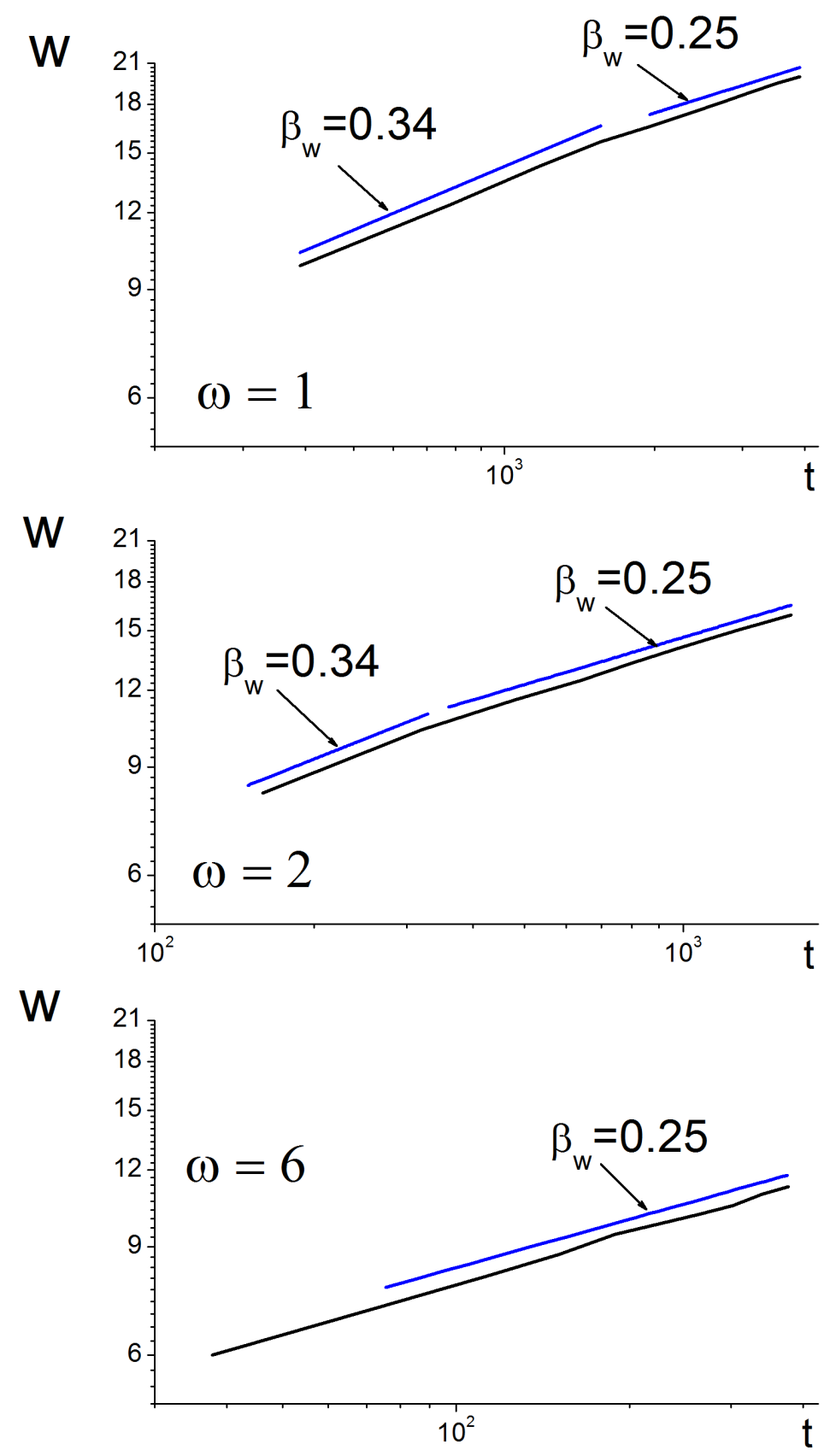

Fig. 13. Time evolution of the width $w(t)$ of the self-correlation peak of the correlation function $K(r, t)$ in the systems we simulated, with $\omega=1,2$, and 6 . 


\section{Chapter 4}

\section{Clusters morphology beyond the classical Young-Laplace model}

\subsection{Introduction}

In this and the following chapter, we develop an analytic theory aimed to explain the results of our Ch. 3 interface dynamics simulations of the cluster coarsening dynamics. Our main goal is to elucidate the effects of the long-range de-wetting interactions on the character of the cluster coarsening dynamics. Our simulations suggest that the long range de-wetting interactions produce significant early time departures from the asymptotic predictions based on the classical Young-Laplace cluster model, Sec. 2.5 .

To understand this effect, as the first step, in this Chapter we provide a mathematical analysis of quasi-static clusters that goes beyond the Young-Laplace model of Sec. 2.5. This model is valid only in the limit of large clusters, i.e., at very long times. In this limit, the detailed form of the de-wetting interaction potential is irrelevant. Indeed, in the Young-Laplace limit, the coarsening exponents are universal, i.e., they do not depend on the potential details such as value of $\omega$ in Eq. (4) of Ch. 2. On the other hand, the simulations of Ch. 3, do evidence significant early time departures from these universal long time coarsening exponents. Moreover, the simulations indicate that these departures are correlated with the long range character of the potential. For example, by the simulations of $\mathrm{Ch}$. 3, for a large $\omega=6$ (essentially, a short range de-wetting potential), the early time range cluster height exponent $\beta_{H}$ is about 0.28 , so it is close to 
the universal asymptotic $\beta_{H}=0.25$. On the other hand, from the Ch. 3 simulations with genuine long-range potentials with for $\omega=1$ and $\omega=2$, the early time range exponent $\beta_{H}$ is about 0.33 , i.e., it deviates significantly from the asymptotic $\beta_{H}=0.25$. It is physically significant to understand these effects. Indeed, the potentials $\omega=1$ and $\omega=2$ are physically interesting cases since they corresponding to van der Waals interaction $(\omega=2)$, and to fermionic Casimir interaction $(\omega=1)$; see Chapters 1 and 2 discussions. In addition, on the experimental side, an effective (early time) exponent for the growth of cluster height, measured to be $\beta_{H}^{\text {eff }} \approx 0.33$, has been indeed reported for Sn clusters on silicon, see Ref. [13] of Ch. 1. Our analytic theory developed in this and the following chapter, in combination with our simulations of $\mathrm{Ch}$. 3, explains these experimental findings in terms of long range de-wetting potentials. The simulations by themselves already indicate that these early time effects are correlated with a breakdown of the Young-Laplace simple model of clusters. For example, within this model, the cluster height $(\mathrm{H})$-to-radius(R) aspect ratio does not depend on the cluster size. Rather, by Eq. (46) of Ch. 2, in the Young-Laplace limit we have,

$$
\frac{2 H}{R} \approx m_{Y}=\tan \left(\theta_{Y}\right)
$$

indicating that the aspect ratio is a constant expressible in terms of the Young angle slope. Likewise, by the discussions of Sec. 2.5, in the Young-Laplace static cluster model for $\mathrm{D}=2$ we have the following simple results for cluster height, cluster base radius, and cluster pressure, expressed as functions of the cluster volume,

$$
H_{\text {stat }}^{\text {Young }}(V)=C_{H} V^{1 / 3}, \quad R_{\text {stat }}^{\text {Young }}(V)=C_{R} V^{1 / 3}, \quad P_{\text {stat }}^{\text {Young }}(V)=C_{P} V^{-1 / 3},
$$

with $C_{H}, C_{R}, C_{P}$ being numerical constants expressible in terms of the Young angle slope; see the Eqs. (65-67) of Ch. 2. However, the clusters seen in the simulations strongly deviate from Young-Laplace behavior in Eqs. (1) and (2). Moreover, in Ch. 3, we have found that these deviations are larger for smaller values of $\omega$, i.e., for longer 
ranged de-wetting interactions. Recall, for example, of the Fig. 7 of Ch. 3, which shows that, in contrast to Eq. (1), the ratio $2 H / R$ is, at early times, significantly smaller than the Young angle slope, especially for smaller values of $\omega$, i.e., for longer ranged de-wetting interactions. As documented in Fig. 7 of Ch. 3, this effect is correlated with the magnitude of the departures of the coarsening exponents from their asymptotic values, which are larger for smaller values of $\omega$.

To quantify these effects, in this chapter we pursue a mathematical discussion of quasi-static clusters shapes and sizes that goes beyond the Young-Laplace model. Our discussion will be used in the following chapter [Ch. 5], to develop a powerful cluster dynamics theory capable to analytically explain the findings of our simulations and related results from the experiments.

\subsection{Quasi-static clusters}

Clusters occurring in our simulations are evolving very slowly and can be considered as (nearly) time-independent, static objects with (nearly) constant pressure. Thus, for $D=2$, the radial profile of a single cluster can be described by Eq. (24) of Ch. 2,

$$
\gamma\left\lfloor\frac{d^{2} h}{d r^{2}}+\frac{1}{r} \frac{d h}{d r}\right\rfloor-\Phi^{\prime}(h)=-P_{\text {stat }},
$$

with $P_{\text {stat }}$ being the static cluster pressure. Here, we will outline the results obtained by solving the Eq. (3), for our rescaled model of Sec. 2.4, with $\gamma=1$, and

$$
\Phi^{\prime}(h)=\frac{1}{h^{\omega+1}}\left\lfloor 1-\left(\frac{h_{\min }}{h}\right)^{\omega}\right\rfloor .
$$

where,

$$
h_{\min }=\left(\frac{\omega+2}{2(2 \omega+1)}\right)^{1 / \omega}
$$


For a given cluster height $H$ (above the dry area, at $h=h_{\text {min }}$; see Fig. $1(\mathrm{c})$ ), we solved the Eq. (3) numerically (using Wolfram's Mathematica), with the boundary conditions,

$$
h(r=0)=H+h_{\min },\left.\frac{d h}{d r}\right|_{r=0}=0 .
$$

For any given $H$, the value of the cluster pressure $P_{\text {stat }}$ in Eq. (3), is chosen such that the $h(r)$ approaches a finite value in the limit of infinite $r$, see Fig. 1(a). Thus, by imposing this boundary condition at infinity, we were able to find the functional relation $P_{\text {stat }}(H)$ expressing the cluster pressure $P_{\text {stat }}$ as a function of the cluster height $H$. For the purposes of Ch. 5, it will be however more convenient to express $P_{\text {stat }}$ as a function of the cluster volume,

$$
V=2 \pi \int_{0}^{R} d r r\left[h(r)-h_{\min }\right]
$$

Here, $R$ is the cluster base radius, fixed by the condition $h(r=R)=h^{*}$ with $h^{*}$ being a characteristic height below which $h(r)$ exponentially approaches its asymptotic value (at infinite $r$ ), see Fig. $1(\mathrm{~b})$. The $h^{*}$ is not a sharply defined quantity. One possible choice would be to identify it as the inflection point of the de-wetting potential $\Phi(h)$. We have however decided for a different convention defining the $h^{*}$, via the condition that the third derivative $\Phi(h)$ vanishes at $h^{*}$. We recall that the same convention is used in Ch. 3 to define clusters. For the rescaled model potential in Eq. (4), this convention gives $h^{*}=1$ for any $\omega$ [as discussed in Sec. 2.4].

By the above described procedure, we have constructed the functional relations,

$$
P_{\text {stat }}(V), H_{\text {stat }}(V), \quad R_{\text {stat }}(V),
$$

expressing cluster pressure, cluster height, and cluster base radius, as functions of the cluster volume. Our numerical results for these functions are given in Figs. 2 through 4 , for the rescaled model with $\omega=1,2$, and 6 . See also the Appendix to this Chapter. 
a)

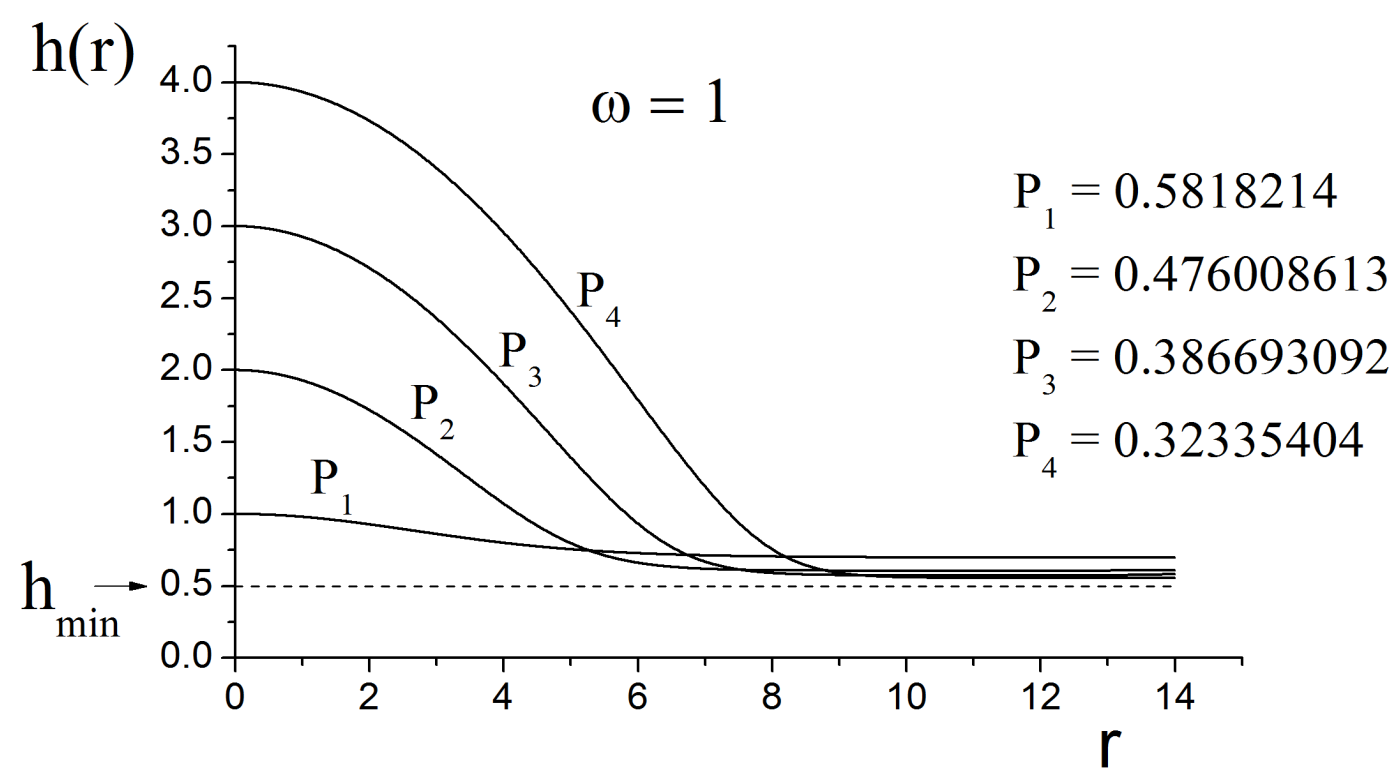

b)

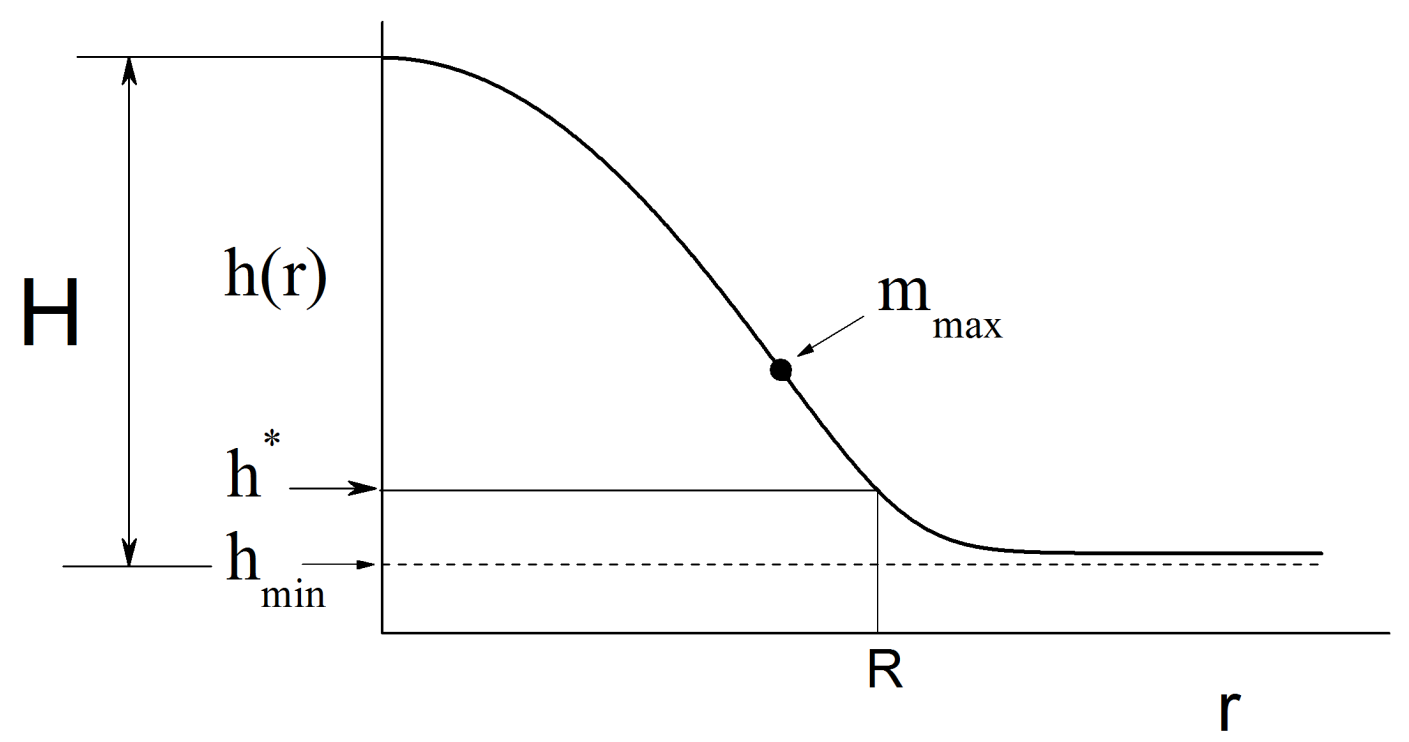

Fig. 1. (a) Cluster profiles obtained by solving the Eq. (3) for $\omega=1$, for several different pressures. To obtain the cluster type solutions, for each cluster height $H$, the pressure is fixed by the boundary condition that the interface height approaches a finite value at infinity. (b) The figure conceptualizes the definition of the cluster radius $R$. Also labeled is the inflection point of the cluster profile at which the interface slope reaches its maximum value $m_{\max }$. 

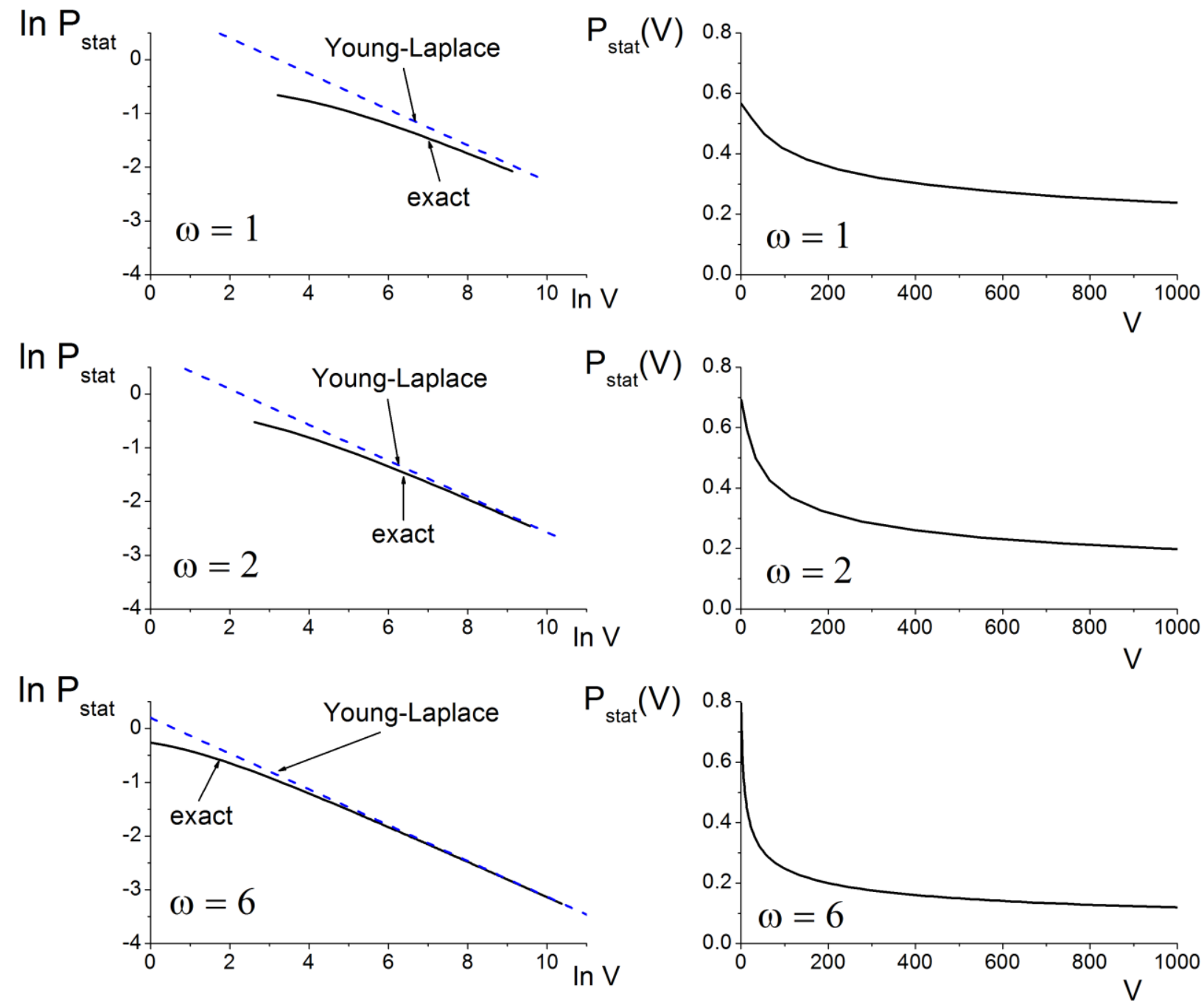

Fig. 2. Our numerical results for the function $P_{\text {stat }}(V)$ for the rescaled model with $\omega=1,2$, and 6 . The dashed line is the corresponding result in the Young-Laplace model; see Eqs. 65-67 of Ch. 2. 

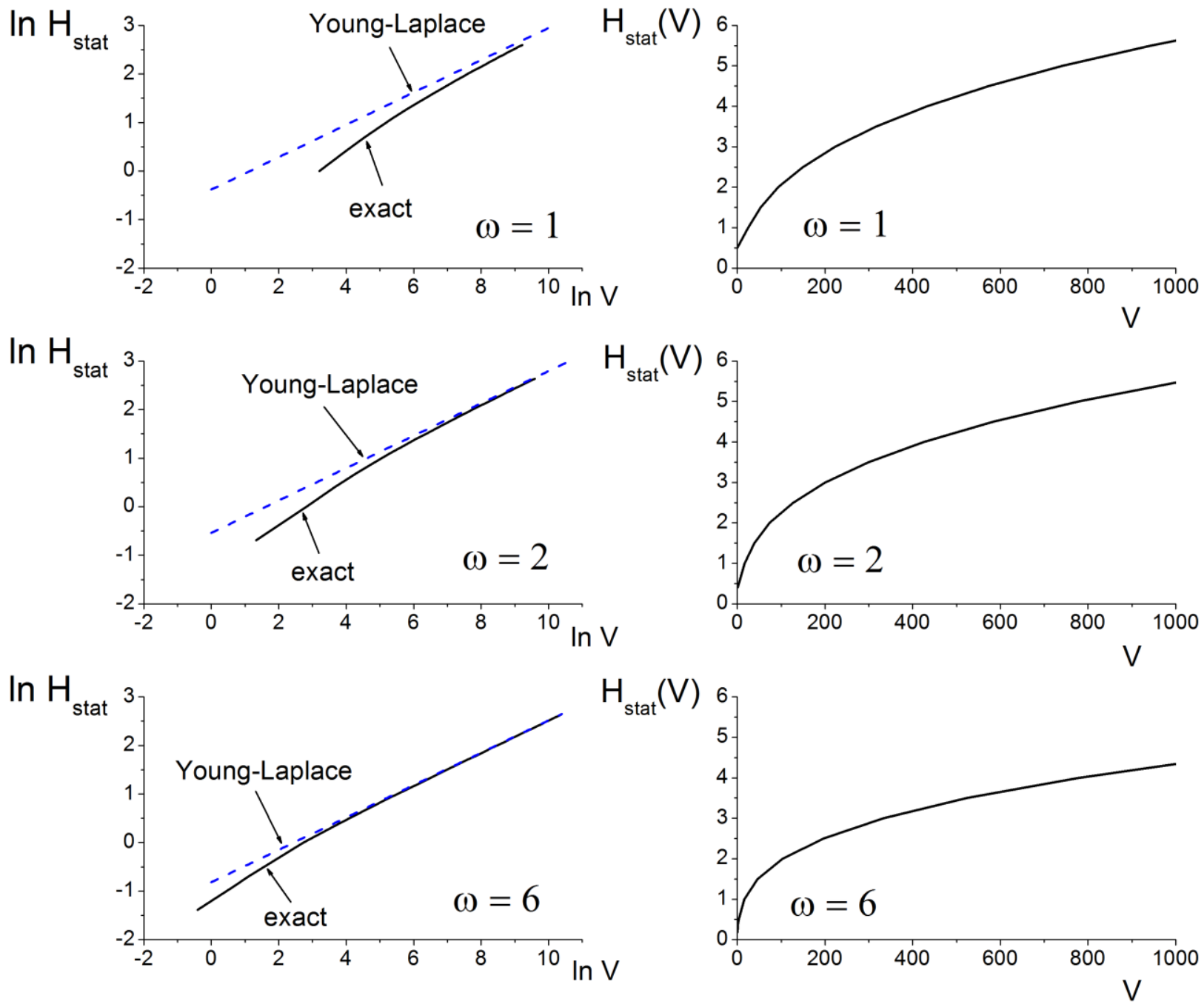

Fig. 3. Our numerical results for the function $H_{\text {stat }}(V)$ for the rescaled model with $\omega=1,2$, and 6 . The dashed line is the corresponding result in the Young-Laplace model; see Eqs. 65-67 of Ch. 2. 

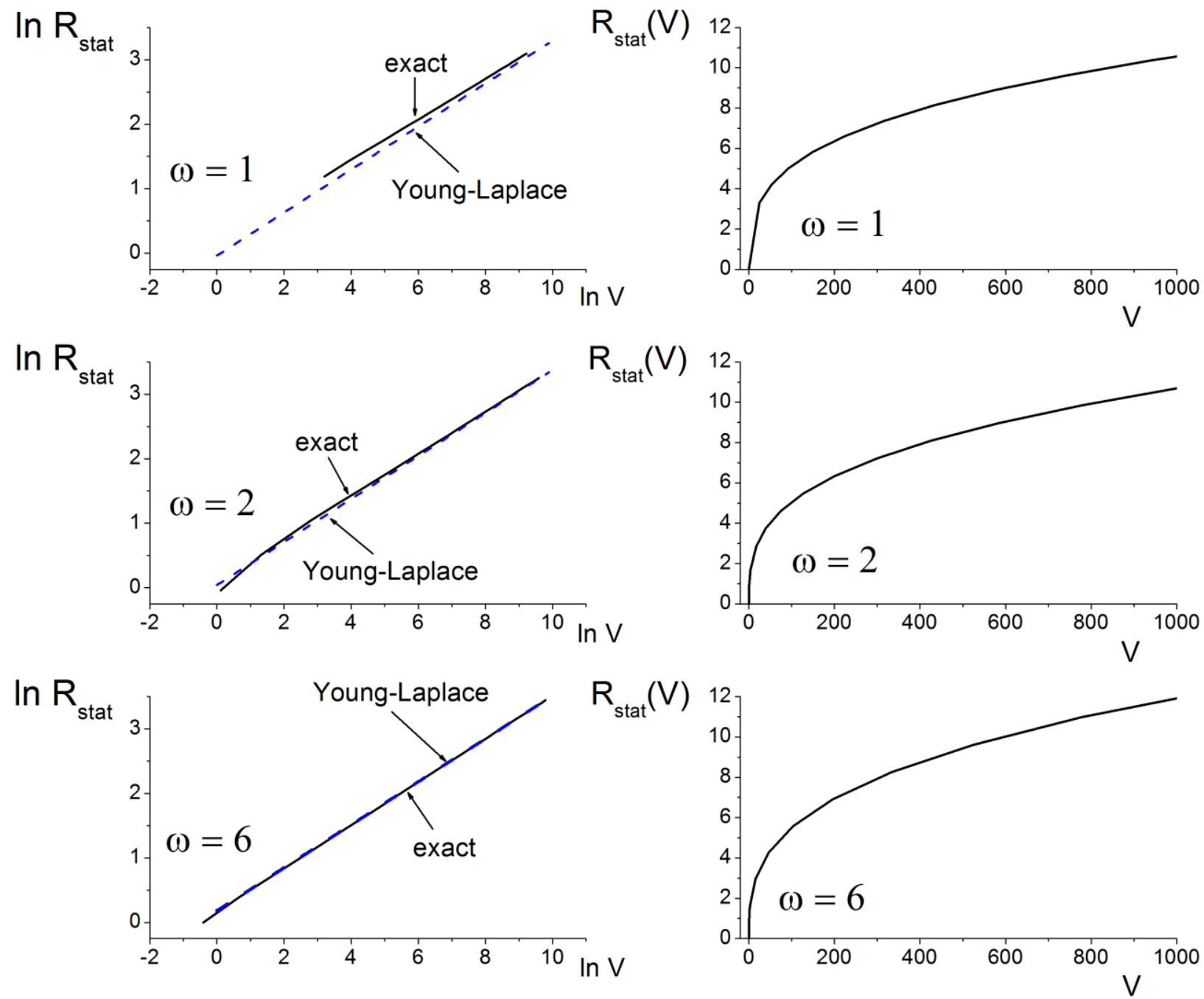

Fig. 4. Our numerical results for the function $R_{\text {stat }}(V)$ for the rescaled model with $\omega=1,2$, and 6 . The dashed line is the corresponding result in the Young-Laplace model; see Eqs. 65-67 of Ch. 2. 
In figure 5 we display several quantities well evidencing the magnitude of the breakdown of the Young-Laplace cluster model. Thus, in Fig. 5 (a), we display the quantity,

$$
\frac{m_{\max }(H)}{m_{Y}},
$$

versus cluster height $H$. Here, $m_{\max }=|\mathrm{dh} / \mathrm{dr}| \max$ is the maximum interface slope occurring at the inflection point of the cluster profile; see Fig. 1(b). The $m_{\max }$ approaches the Young angle slope $m_{Y}$ for large $H$, so the ratio in Eq. (9) approaches 1 in the YoungLaplace limit. However, for a finite $H$, this ratio is smaller than one, and this effect is especially prominent for de-wetting potentials with smaller values of $\omega$ such as $\omega=1$ and $\omega=2$. On the other side, for a large $\omega=6$ (essentially, a short range potential), the ratio in Eq. (9) quickly approaches 1, i.e., the Young-Laplace limit. The same trend is seen in other quantities displayed in Fig. 5 for various values of $\omega$. Thus, in Fig. 5(b) we display the ratio,

$$
\frac{\frac{2 H}{R_{\text {stat }}(H)}}{m_{Y}} \text {, }
$$

which by Eq. (1) approaches 1 in the Young-Laplace limit of infinite cluster height $H$. We also display, in Fig. 5(c),

$$
\frac{P_{\text {stat }}(H)}{P_{\text {staut }}^{\text {Young }}(H)}
$$

being the ratio between the true pressure of a cluster with height $H$, and the YoungLaplace model pressure for a cluster of the same height $H$ [given by Eq. (47) of Ch2]. Finally we display, in Fig. 5(d), the quantity

$$
\frac{V_{\text {stat }}^{\text {Young }}(H)}{V_{\text {stat }}(H)}
$$


a)

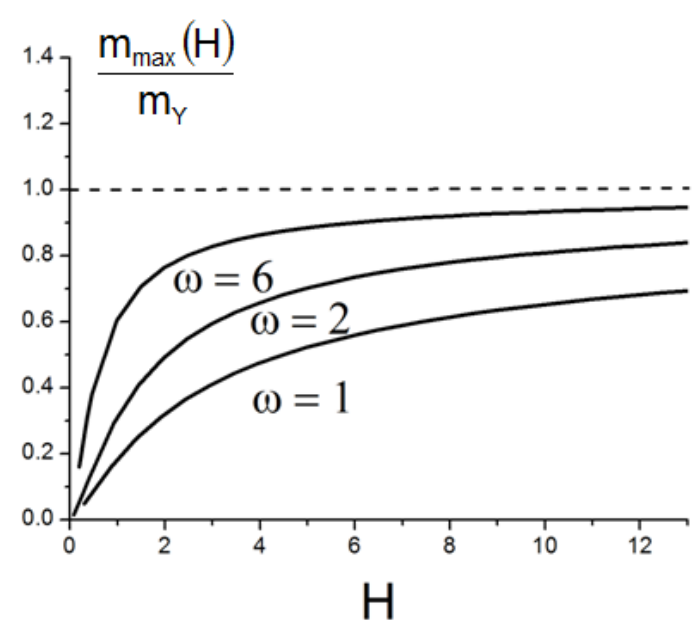

c)

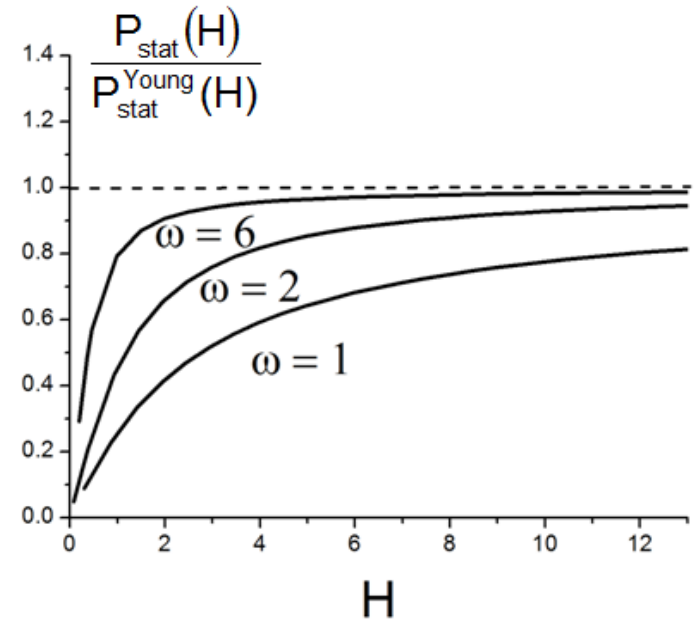

b)

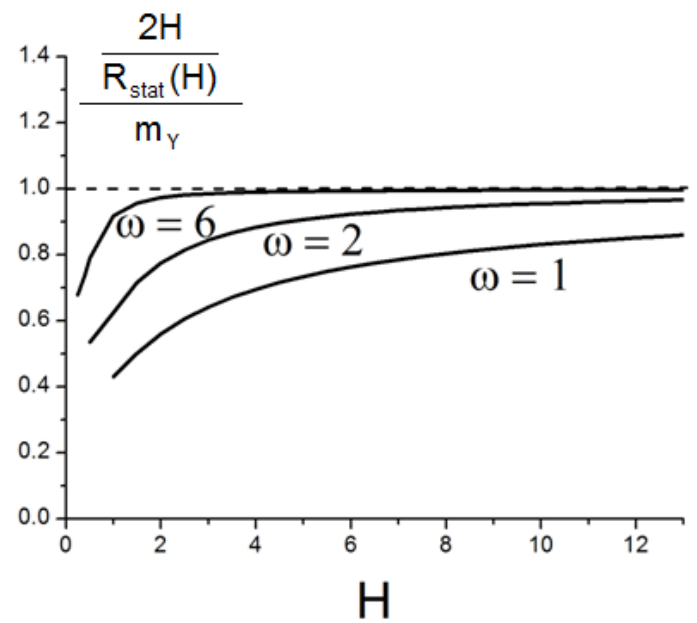

d)

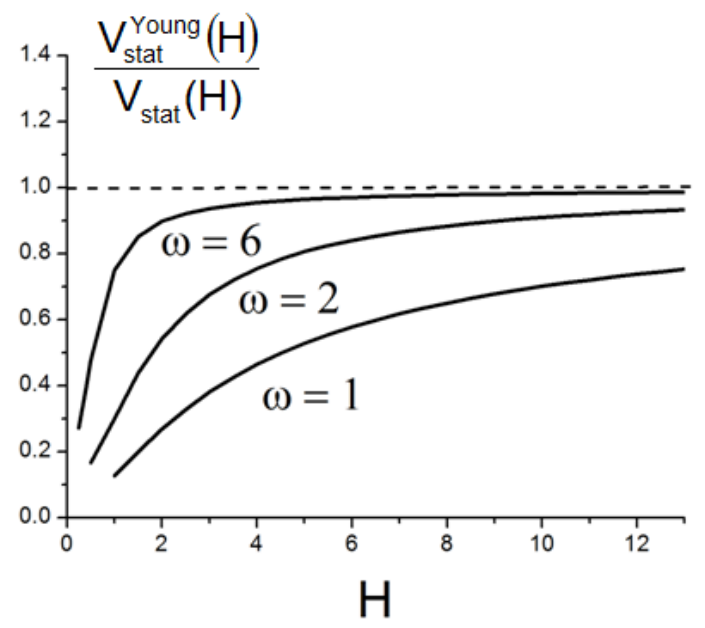

Fig. 5. The figure documents the way how the four cluster properties in Eqs. (9) through (12) approach the Young-Laplace limit with increasing cluster height $H$, for the rescaled model with $\omega=1,2$, and 6 . 
being the ratio between the Young-Laplace model volume of the cluster with the height $H$ and the true volume of the cluster with the same height $H$. The ratios in Eqs. (10-12) all approach 1 in the limit of infinite cluster height $H$. However, for a finite $H$, these ratios are all smaller than one, and this effect is especially prominent for de-wetting potentials with smaller values of $\omega$ such as $\omega=1$ and $\omega=2$. On the other side, for a large $\omega=6$ (essentially, a short range potential), all these ratios in Eqs. (10-12) quickly approach 1, i.e., the Young-Laplace limit.

The results in Fig. 5 are in accord with the Ch. 3 simulations results indicating that the deviations from the Young-Laplace model are larger for smaller values of $\omega$, i.e., for longer ranged de-wetting interactions. Thus, for example, the Fig. 7 of Ch. 3 shows that, in contrast to Eq. (1) but in accord with Fig. $5(\mathrm{~b})$, the ratio $2 H / R$ is, at early times, significantly smaller than the Young angle slope, especially for smaller values of $\omega$, i.e., for longer range de-wetting interactions. As documented in Fig. 7 of Ch. 3, this effect is correlated with the magnitude of the departures of the coarsening exponents from their asymptotic values, which are larger for smaller values of $\omega$, i.e., for the longer range interactions. This dynamical effect will be discussed in the next chapter [Ch. 5], in which the results of the present chapter are used as inputs to a novel cluster dynamics theory capable to analytically explain the results of $\mathrm{Ch} .3$ simulations as well as the related results from the experiments such as those of Ref. [13] of Ch. 1. 


\subsection{Appendix}

The functions $P_{\text {stat }}(V), H_{\text {stat }}(V), R_{\text {stat }}(V)$ displayed in Figs. 2 through 4 are obtained on a discrete set of $V$ points involved in the procedure discussed in Sec. 4.2. However, for the purposes of Ch. 5, we need these functions as functions of a continuous $V$. For this purpose, we fitted the discrete $V$ set functions by suitably chosen continuous $V$ functions. Rather than using $P, H$, and $R$ as functions of $V$, in the mathematical framework of Ch. 5 it is more natural to employ a change of variables and express

$$
\bar{P}=\ln (P), \bar{H}=\ln (H), \quad \bar{R}=\ln (R),
$$

as functions of

$$
\bar{V}=\ln (V) .
$$

We fitted our discrete data with the 4-parameter fits of the form,

$$
\begin{aligned}
& \bar{P}=A_{P}^{(0)}+A_{P}^{(1)} \bar{V}+\left(B_{P}^{(0)}+B_{P}^{(1)} \bar{V}\right) \exp (-\bar{V} / 3), \\
& \bar{H}=A_{H}^{(0)}+A_{H}^{(1)} \bar{V}+\left(B_{H}^{(0)}+B_{H}^{(1)} \bar{V}\right) \exp (-\bar{V} / 3), \\
& \bar{R}=A_{R}^{(0)}+A_{R}^{(1)} \bar{V}+\left(B_{R}^{(0)}+B_{R}^{(1)} \bar{V}\right) \exp (-\bar{V} / 3) .
\end{aligned}
$$

In Eq. (A3), the terms with the A-constants are motivated by the Young-Laplace large-V limit; see the equations 65 through 67 of Ch. 2. In this limit, the terms in (A3) with the Bconstants would represent the asymptotic corrections to the leading order terms, while, by the Eqs. 65-67 of Ch. 2, the A-constants in the leading order terms in (A3) would have to be fixed to 


$$
\begin{aligned}
& A_{P}^{(1)}=-\frac{1}{3}, A_{H}^{(1)}=\frac{1}{3}, \quad A_{R}^{(1)}=\frac{1}{3} \\
& A_{P}^{(0)}=\ln \left(C_{P}\right), A_{H}^{(0)}=\ln \left(C_{H}\right), A_{R}^{(0)}=\ln \left(C_{R}\right) .
\end{aligned}
$$

Thus, in the large-V limit, the A-constants are fixed and the Eqs. (A3) reduce to 2parameters fits, for the B-constants only. On the other hand, in our Ch. 5 analytic calculations, we will be interested to compare analytic results with the results obtained from the simulations of $\mathrm{Ch}$. 3 . In these simulations, the cluster volumes are below the large-V range in which Eq. (A4) applies. For this reason, the Eqs. (A3) are considered as true 4-parameter fits that are done in the finite V-range actually encountered in the simulations. Using this procedure we have obtained excellent fits used in the Chapter 5 calculations. 


\section{Chapter 5}

\section{Analytic Theory of the Cluster Coarsening Growth}

\subsection{Introduction}

In Chapter 3, we have used numerical simulations of our interface dynamics model to explore the role played by the long-range de-wetting interactions on the character of the cluster coarsening dynamics. Main physical effect suggested by our simulations is that the long range de-wetting interactions produce significant early time departures from the asymptotic predictions based on the classical Young-Laplace model. To address this phenomenon, as the first step, in Chapter 4 we have provided a theoretical analysis of quasi-static clusters that goes beyond the Young-Laplace model.

In this Chapter, we will use the results of this analysis to develop a powerful cluster dynamics theory capable to analytically explain the findings of our simulations. Main result of this chapter is that the effective cluster growth coarsening exponents can be expressed as functions of the cluster size. In this chapter we will calculate these exponents by our analytic approach, and compare them with the results obtained from our numerical simulations of Chapter 3. We find a very good agreement between our analytic results and the corresponding results for the effective exponents obtained from our simulations. This agreement is the final proof of our assertion that the long range de-wetting interactions are responsible for the significant early time departures from the asymptotic predictions based on the classical Young-Laplace model. 


\subsection{Cluster dynamics and statistics}

We begin our discussion by considering in more detail the dynamics of clusters. A simple model for this dynamics was introduced already in Sec. 2.5. By the Eqs. (48) and $\left(52^{\prime}\right)$ of $C h .2$, we have [for $D=2$ ] the following result for the dynamics of a cluster cluster with volume $V$,

$$
\frac{d V}{d t}=-2 \pi \Gamma \Omega \frac{P(R)-P(\xi)}{\ln (\xi / R)}
$$

By the discussions of Sec. 2.5, the $P(R)$ in Eq. (1) can be identified with the quasi-static cluster pressure, expressible [by the results of Ch. 4; see Fig. 2 therein] as a function of the cluster volume, labeled as $P_{\text {stat }}(V)$. In addition, by the Ch. 4 discussions [see Fig. 4 therein], the cluster radius entering Eq. (1) is also expressible as a function of cluster volume, $R_{\text {stat }}(V)$. We will handle the Eq. (1) in the spirit of the statistical mean-field Lifhits-Slyozov theory [1-3], so the Eq. (1) will be replaced by the equation

$$
\frac{d V}{d t}=F(V)=-2 \pi \Gamma \Omega \frac{P_{\text {stat }}(V)-P^{*}}{\ln \left[\xi / R_{\text {stat }}(V)\right]} .
$$

In Eq. (2), the length-scale $\xi$ is a "screening length" proportional to the average intercluster separation $\lambda$, as discussed in Sec. 2.5. The volume conservation law in Eq. (1) of Ch. 2 ensures that the average cluster volume is equal to film volume initially (at $t=0$ ) covering the area $=\pi(\lambda / 2)^{2}$. Thus we have,

$$
<V>=\pi\left(\frac{\lambda}{2}\right)^{2} \tilde{h}_{0}
$$

where $\tilde{h}_{0}$ labels the initial film thickness. By Eq. (3), we can write,

$$
\xi=C \lambda=C \sqrt{\frac{4<V>}{\pi \tilde{h}_{0}}},
$$


where $C$ is a numerical proportionality constant.

Within the Lifhits-Slyozov statistical approach, we will describe the ensemble of clusters by their volume distribution function $\rho(V, t)$ such that $\rho(V, t) d V=d N=$ the number of clusters within the volume interval $(V, V+d V)$. Thus, the total number of clusters is,

$$
N=\mathrm{\jmath} d N=\mathrm{\jmath} d V \rho(V, t)
$$

The total film volume is

$$
V_{f i l m}=\mathrm{\jmath} d N V=\mathrm{\jmath} d V \rho(V, t) V .
$$

Thus, the average cluster volume is given by

$$
<V>=\frac{V_{\text {film }}}{N}=\int d V \pi(V, t) V .
$$

Here,

$$
\pi(V, t)=\frac{\rho(V, t)}{N}
$$

is the cluster volume probability density function. The time evolution of the cluster distribution function is, in general, governed by the continuity equation,

$$
\frac{\partial}{\partial t} \rho(V, t)=-\frac{\partial}{\partial V}[F(V) \rho(V, t)]
$$

Here, in our case, the function $F(V)$ is given by Eq. (2). The conservation of the total film volume Eq. (6), implies a restriction on the possible form of $F(V)$ that can be used to fix the value of the parameter $P^{*}$ introduced in Eq. (2). Indeed, by using Eqs. (6) and (9), it is easy to show that the condition $d V_{\text {film }} / d t=0$ implies the condition

$$
0=<F(V)>=\int d V \pi(V, t) F(V)
$$


In combination with the form of $F(V)$ in Eq. (2), the Eq. (10) can be easily used to obtain the value of the parameter $P^{*}$.

\subsection{Cluster distribution cut off}

A special feature of the here interesting cluster distributions is that they exhibit a sharp cut off $V_{\max }(t)$ (the maximum cluster size) such that

$$
\rho(V, t)=0, \text { for } V>V_{\max }(t) .
$$

This cut off will play an essential role in the following discussions. Within the present kinetic theory Eq. (9), the presence of the sharp distribution cut off imposes a special condition, the solvability condition revealed by Lifhits and Slyozov, of the form

$$
\left(F(V)-V \frac{\partial F(V)}{\partial V}\right)_{V=V_{\max }(t)}=0,
$$

which is easily seen to be equivalent to

$$
\left.\frac{\partial \ln [F(V)]}{\partial \ln (V)}\right|_{V=V_{\max }}=1 .
$$

A consequence of the condition Eq. (12) is that the cut off size dynamics equation,

$$
\frac{d V_{\max }}{d t}=F\left(V_{\max }\right)
$$

can be written also as,

$$
\frac{d V_{\max }}{d t}=\left(V \frac{\partial F(V)}{\partial V}\right)_{V=V_{\max }} .
$$


To proceed with discussing the dynamics of $V_{\max }(t)$, we will simplify our kinetic theory by using the approximation (valid for $V \sim V_{\max }$ ),

$$
F(V)=-2 \pi \Gamma \Omega \frac{P_{\text {stat }}(V)-P^{*}}{\ln \left[\xi / R_{\text {stat }}(V)\right]} \approx-2 \pi \Gamma \Omega \frac{P_{\text {stat }}(V)-P^{*}}{\ln \left[\xi / R_{\text {stat }}\left(V_{\text {max }}\right)\right]},
$$

within which the Eq. (10) yields the result

$$
P^{*}=<P_{\text {stat }}(V)>，
$$

while the Eq. (12) reduces to

$$
P^{*}=P_{\text {stat }}\left(V_{\max }\right)-V_{\text {max }} \frac{d P_{\text {stat }}\left(V_{\max }\right)}{d V_{\max }} .
$$

By Eqs. (15) and (14), we obtain an equation giving the dynamics of $V_{\max }(t)$, of the form,

$$
\frac{d V_{\max }}{d t}=\frac{V_{\max }}{\tau\left(V_{\max }\right)}
$$

with conveniently defined $V_{\max }$ - dependent "time constant" defined via,

$$
\frac{1}{\tau\left(V_{\max }\right)}=2 \pi \Gamma \Omega \frac{-\left(\frac{d P_{\text {stat }}(V)}{d V}\right)_{V=V_{\max }}}{\ln \left(\frac{\xi}{R_{\text {stat }}\left(V_{\max }\right)}\right)} .
$$

We recall that, by Eq. (4), the length-scale $\xi$ in the above equation is given by,

$$
\xi=C \lambda=C \sqrt{\frac{4<V>}{\pi \tilde{h}_{0}}} .
$$

Eqs. (18) through (20) will form a closed description of the dynamics of $V_{\max }$ provided we manage to express the $\langle V\rangle$ [entering Eq. (20)] as a function $V_{\max }$. If this closing is achievable, then the right hand side of the first order differential equation (18) would 
become a function of $V_{\max }$ only. Such an equation can be then directly integrated. The result would give the time evolution of $V_{\max }$.

\subsection{Closing the cut off dynamics equation}

Fortunately, the above anticipated closing of the cut off dynamics equation (18) is indeed possible. We base it on the approximate relation

$$
<P_{\text {stat }}(V)>\approx P_{\text {stat }}(<V>)
$$

For our system, the relation in Eq. (21) is indeed satisfied to a very good approximation. We document this in Fig. 1 from our simulations, which plots the obtained average cluster pressure $\langle P\rangle$ versus the average cluster volume $\langle V\rangle$, obtained at various times. In the same figure, we give also the function $P_{\text {stat }}(V)$ as obtained in our analytic calculations discussed in Chapter 4 [see the Fig. 2 therein]. From the Fig. 1 one can see that Eq. (21) holds for essentially all times.

By Eq. (21) in combination with Eqs. (16) and (17), we obtain the relation

$$
P^{*}=P_{\text {stat }}(<V>)=P_{\text {stat }}\left(V_{\max }\right)-V_{\max } \frac{d P_{\text {stat }}\left(V_{\max }\right)}{d V_{\max }},
$$

i.e.,

$$
P^{*}=P_{\text {stat }}(<V>)=P_{\text {stat }}\left(V_{\max }\right) \cdot\left\lfloor 1-\frac{d \ln \left[P_{\text {stat }}\left(V_{\max }\right)\right]}{d \ln \left(V_{\max }\right)}\right\rfloor
$$

By Eq. (22),

$$
<V>=P_{\text {stat }}^{-1}\left[P_{\text {stat }}\left(V_{\max }\right)-V_{\max } \frac{d P_{\text {stat }}\left(V_{\max }\right)}{d V_{\max }}\right\rfloor .
$$

Here, the $P_{\text {stat }}{ }^{-1}$ signifies the inverse function of the function $P_{\text {stat }}(V)$. Note that by Eq. (23), we managed to express the average cluster volume $\langle V\rangle$ [entering the Eq. (20)] as a function $V_{\max }$. 
a)

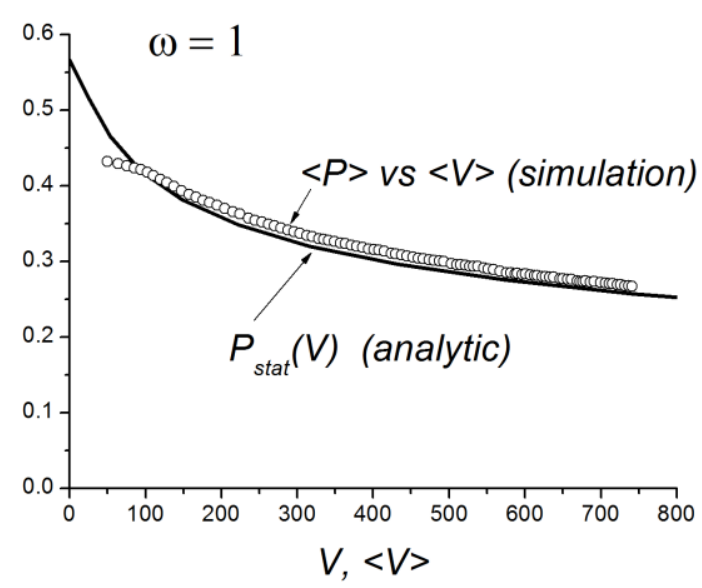

b)

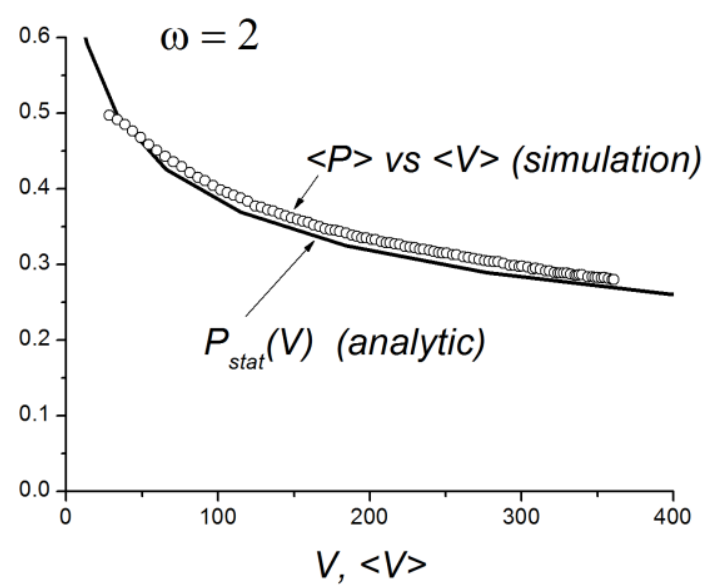

c)

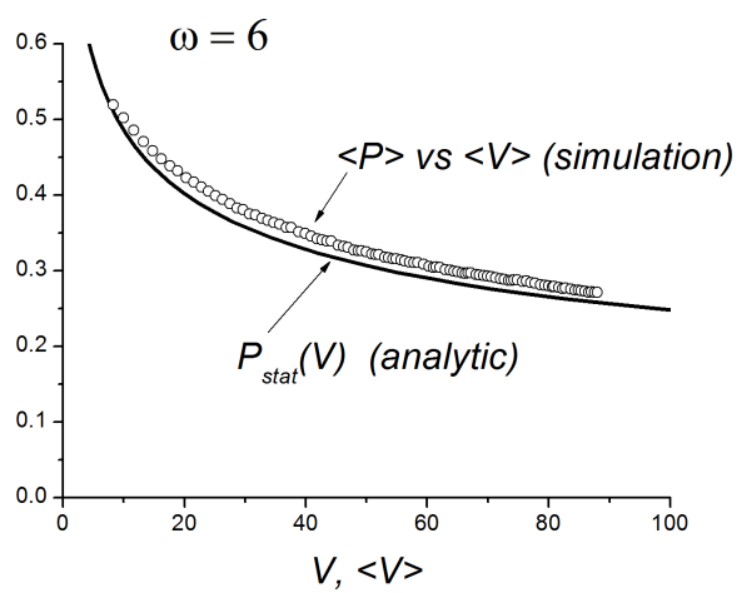

Fig. 1: (a) The figure contains two plots. One of them is obtained from our simulations of the model with $\omega=1$, in $D=2$. It plots the obtained average cluster pressure $\langle\mathrm{P}>$ versus the average cluster volume $\langle\mathrm{V}\rangle$, obtained at various times. In the same figure, we give also the plot displaying the function $P_{\text {stat }}(V)$ as obtained in our analytic calculations in Chapter 4 , for $\omega=1$, in $D=2$ [see also Fig. 2 of Ch.4]. From the displays of the two plots, one can see that Eq. (21) holds for essentially all times [except for very early times, when the clusters are small and not quasi-static]. The figure (b) is the same as the figure (a) but for the model with $\omega=2$, in $D=2$. The figure (b) is the same as the figure (a) but for the model with $\omega=6$, in $D=2$. 
In combination with Eq. (23), the Eqs. (18) through (20) form a closed description for the dynamics of $V_{\max }$. The right hand side of the first order differential equation (18) thus becomes a function of $V_{\max }$ only and can be thus integrated. The result of this integration gives the time evolution of $V_{\max }$ through the integral,

$$
t=\int^{V_{\max }} \frac{d V}{V} \tau(V)=\int^{V_{\max }} d \ln (V) \tau(V),
$$

Here, by Eqs. (19), (20), and (23),

$$
\tau(V)=\left[2 \pi \Gamma \Omega \frac{-\frac{d P_{\text {stat }}(V)}{d V}}{\ln \left(\frac{\xi(V)}{R_{\text {stat }}(V)}\right)}\right]^{-1},
$$

with

$$
\xi(V)=C \sqrt{\frac{4 P_{\text {stat }}^{-1}\left[P_{\text {stat }}(V)-V \frac{d P_{\text {stat }}(V)}{d V}\right]}{\pi \tilde{h}_{0}}} .
$$

The equation (24) gives the system evolution time $t$ as a function of $V_{\max }$. Inverting this function thus gives the $V_{\max }$ as function of the time $t$. Thus, the Eq. (24), in combination with Eqs. (25) and (26), provide the desired solution $V_{\max }(t)$ for the evolution of the cluster distribution cut off, i.e., of the maximum cluster size.

\subsection{Calculating the effective coarsening exponents}

A neat feature of our equation (24) is that it can be used to obtain the effective exponents of the cluster coarsening growth. Interestingly, as discussed in the following, these effective coarsening exponents can be expressed as functions of cluster size, e.g., 
as functions of the cluster average volume $\langle V\rangle$ at any time $t$. This is one of the major results of this thesis.

To derive this significant result, we first discuss the effective coarsening exponent for the growth of the cut off, i.e., the maximum cluster size $V_{\max }(t)$,

$$
\beta_{V_{\max }}=\frac{d \ln \left(V_{\max }\right)}{d \ln (t)} .
$$

By Eq.(27),

$$
\beta_{V_{\max }}=\frac{1}{\frac{d \ln (t)}{d \ln \left(V_{\max }\right)}}=\frac{t}{\frac{d t}{d \ln \left(V_{\max }\right)}} .
$$

Using here Eq. (24), we obtain,

$$
\beta_{V_{\max }}=\frac{\int^{V_{\max }} d \ln (V) \tau(V)}{\tau\left(V_{\max }\right)} .
$$

By Eq. (28), it is easy to see that,

$$
\frac{d}{d \ln \left(V_{\max }\right)}\left[\tau\left(V_{\max }\right) \beta_{V_{\max }}\right]=\tau\left(V_{\max }\right) .
$$

After a little algebra, Eq. (29) can be transformed into the differential equaton,

$$
\frac{d \beta_{V_{\max }}}{d \ln \left(V_{\max }\right)}+\frac{1}{\beta_{V_{\max }}^{(0)}} \beta_{V_{\max }}=1,
$$

with,

$$
\frac{1}{\beta_{V_{\max }}^{(0)}}=\frac{d \ln \left[\tau\left(V_{\max }\right)\right]}{d \ln \left(V_{\max }\right)} .
$$


Note that solving the differential equation (30) effectively gives the desired effective coarsening $\beta_{V_{\max }}$ expressed as function of $V_{\max }$. It is convenient to re-express Eq. (30) as,

$$
\beta_{V_{\max }}=\beta_{V_{\max }}^{(0)}\left(V_{\max }\right) \cdot\left\lfloor 1-\frac{d \beta_{V_{\max }}}{d \ln \left(V_{\max }\right)}\right\rfloor
$$

The $\beta_{V_{\max }}$ and other effective coarsening exponents are slowly changing functions of $V_{\max }$ [as documented in the following sections], so the Eq. (32) can be solved iteratively, by writing,

$$
\beta_{V_{\max }}^{(n)}\left(V_{\max }\right)=\beta_{V_{\max }^{(0)}}^{(0)}\left(V_{\max }\right) \cdot\left\lfloor 1-\frac{d \beta_{V_{\max }}^{(n-1)}\left(V_{\max }\right)}{d \ln \left(V_{\max }\right)}\right\rfloor, \quad n=1,2,3, \ldots
$$

with $\beta_{V_{\max }}^{(0)}$ in Eq. (31) being the zero-th order approximation. From the concrete calculations discussed in Sec. 5.7, we find that the Eq. (33) is a rapidly converging iteration. The effective coarsening exponent

$$
\beta_{V_{\max }}\left(V_{\max }\right)=\lim _{n \rightarrow \infty} \beta_{V_{\max }^{(n)}}^{(n)}\left(V_{\max }\right)
$$

is well approximated already by $\beta_{V_{\max }}^{(n=3)}\left(V_{\max }\right)$. Importantly, by the above discussions, thus obtained effective coarsening exponent $\beta_{V_{\max }}$ is expressed as a function of $V_{\max }$. This exponent can be expressed also as a function of the average cluster volume $\langle V\rangle$. This can be accomplished by using the Eq. (23) relating the $\langle V\rangle$ to $V_{\max }$. In practice, this can be done by making the parametric plot of $\beta_{V_{\max }}$ versus $\langle V\rangle$, with both of these variables expressed as functions of $V_{\max }$.

Once the $\beta_{V_{\max }}$ is calculated, other coarsening exponents can be also calculated in a straightforward fashion. Thus, the exponent for the growth of the average cluster volume,

$$
\beta_{<V>}=\frac{d \ln (<V>)}{d \ln (t)}
$$


can be expressed as,

$$
\beta_{<V>}=\frac{d \ln (<V>)}{d \ln \left(V_{\max }\right)} \cdot \frac{d \ln \left(V_{\max }\right)}{d \ln (t)},
$$

i.e.,

$$
\beta_{<V>}=\frac{d \ln (<V>)}{d \ln \left(V_{\max }\right)} \cdot \beta_{V_{\max }}\left(V_{\max }\right) .
$$

Here, the first term is a function of $V_{\max }$ that can be calculated using Eq. (23) expressing $<V>$ as function of $V_{\max }$.Thus, the Eq. (37) gives the exponent $\beta<v>$ as function of $V_{\max }$. It is however more natural to express this exponent as a function of the average cluster volume $\langle V\rangle$. This can be accomplished by using the Eq. (23) relating the $\langle V\rangle$ to $V_{\max }$. In practice, this can be done by making the parametric plot of $\beta_{<V\rangle}$ versus $\langle V\rangle$, with both variables expressed as functions of $V_{\max }$.

Within our theory, it is also possible to calculate the exponents associated with the coarsening dynamics of other quantities characterizing the ensemble of clusters, such as average cluster pressure, cluster height, and cluster base radius. Thus, for the average cluster pressure we have, by Eq. (21),

$$
<P>=<P_{\text {stat }}(V)>\approx P_{\text {stat }}(<V>),
$$

So, the pressure exponent,

$$
\beta_{<P>}=\frac{d \ln (<P>)}{d \ln (t)} \approx \frac{d \ln \left[P_{\text {stat }}(<V>)\right]}{d \ln (t)},
$$

can be calculated as,

$$
\beta_{<P>}=\frac{d \ln \left[P_{\text {stat }}(<V>)\right]}{d \ln (<V>)} \cdot \frac{d \ln <V>}{d \ln (t)},
$$

i.e.,

$$
\beta_{<P>}=\frac{d \ln \left[P_{s t a t}(<V>)\right]}{d \ln (<V>)} \cdot \beta_{<V>} .
$$


In Eq. (40), the $\beta_{<V>}$ is calculated as described before, while the first term can be calculated by using the results of Ch. 4 for the pressure $P_{\text {stat }}(V)$ of the quasi-static clusters. In effect, the equation (40) thus gives the exponent $\beta_{<p>}$ expressed as a function of the cluster average volume $\langle V\rangle$.

The coarsening exponents for growth of average cluster height and base radius are obtained using the relations

$$
<H>\approx H_{\text {stat }}(<V>), \quad<R>\approx R_{\text {stat }}(<V>),
$$

with the functions $H_{\text {stat }}$ and $R_{\text {stat }}$ calculated in Ch.4 [see the Figs. 3 and 4 therein]. By Eq. (41),

$$
\beta_{<H>}=\frac{d \ln (<H>)}{d \ln (t)} \approx \frac{d \ln \left[H_{\text {stat }}(<V>)\right]}{d \ln (<V>)} \cdot \frac{d \ln <V>}{d \ln (t)}=\frac{d \ln \left[H_{\text {stat }}(<V>)\right]}{d \ln (<V>)} \cdot \beta_{<V>}
$$

and,

$$
\beta_{<R>}=\frac{d \ln (<R>)}{d \ln (t)} \approx \frac{d \ln \left[R_{\text {stat }}(<V>)\right]}{d \ln (<V>)} \cdot \frac{d \ln <V>}{d \ln (t)}=\frac{d \ln \left[R_{\text {stat }}(<V>)\right]}{d \ln (<V>)} \cdot \beta_{<V>} .
$$

Thus, once the exponent $\beta_{<v>}$ is calculated (as described before), the Eqs. (42) and (43) can be then used to calculate the exponents for the growth of the cluster average height and base radius. In Sec. 5.7, we will employ the theory we developed in this and prevous sections of this chapter to derive various coarsening exponents for the clusters emerging in our interface dynamics model, Ch. 2, with the functions $P_{\text {stat }}(V), H_{\text {stat }}(V)$, and $R_{\text {stat }}(V)$ calculated in Ch. 4 [see the figures 2-4 therein]. Prior to this, in the next section, we will first revisit the Young-Laplace model, Sec. 2.5.

\subsection{Young-Laplace model revisited}

As an important illustration for the theoretical program developed in the revious section of this chapter, we will first apply it to Young-Laplace model discussed in Sec. 2.5 of Ch.2. This model is interesting in its own right since it gives asymptotic behavior 
of the coarsening process at the longest time scales. In the Young-Laplace limit, we have (for $D=2$ ),

$$
H_{\text {stat }}^{\text {Young }}(V)=C_{H} V^{1 / 3}, \quad R_{\text {stat }}^{\text {Young }}(V)=C_{R} V^{1 / 3}, \quad P_{\text {stat }}^{\text {Young }}(V)=C_{P} V^{-1 / 3}
$$

with $C_{H}, C_{R}, C_{P}$ being numerical constants expressible in terms of the Young angle slope; see Eqs. $65-67$ of Ch. 2. By Eq. (44), in combination with Eq. (22') we have,

$$
<V>^{-1 / 3}=V_{\max }^{-1 / 3} \cdot[1-(-1 / 3)]
$$

i.e.,

$$
<V>=\left(\frac{3}{4}\right)^{3} V_{\max } .
$$

Next, using the Eq. (44) in combination with Eqs. (25) and (26), we have

$$
\tau(V)=A V^{4 / 3} \ln \left(K \frac{V^{1 / 3}}{\tilde{h}_{0}}\right),
$$

with the numerical constants,

$$
K=\frac{4}{\pi}\left(\frac{3}{4}\right)^{3} \frac{C^{2}}{C_{R}^{2}}, \quad A=\frac{3}{2 C_{P}} \cdot \frac{1}{2 \pi \Gamma \Omega} .
$$

By Eqs. (46) and (28), after an elementary integration, we find,

$$
\beta_{V_{\max }}=\frac{3}{4} \cdot\left(1-\frac{1}{4 \cdot \ln \left[K V_{\max }^{1 / 3} / \tilde{h}_{0}\right]}\right)
$$

or, by Eq.(45),

$$
\beta_{V_{\max }}=\frac{3}{4} \cdot\left(1-\frac{1}{4 \cdot \ln \left[(4 K / 3)<V>^{1 / 3} / \tilde{h}_{0}\right]}\right) .
$$

By Eq. (45), we have,

$$
\beta_{<V>}=\beta_{V_{\max }} .
$$


Thus, by Eq. (49),

$$
\beta_{<V>}=\frac{3}{4} \cdot\left(1-\frac{1}{4 \cdot \ln \left[(4 K / 3)<V>^{1 / 3} / \tilde{h}_{0}\right]}\right) .
$$

Next, by Eqs. (40), (42), and (43), in combination with the Eq. (44), we have,

$$
\beta_{<P>}=-\frac{1}{3} \beta_{\langle V\rangle}, \quad \beta_{H}=\beta_{R}=\frac{1}{3} \beta_{\langle V\rangle} .
$$

Thus, by Eqs. (51) and (52), we find,

$$
\beta_{<P>}=-\frac{1}{4} \cdot\left(1-\frac{1}{4 \cdot \ln \left[(4 K / 3)<V>^{1 / 3} / \tilde{h}_{0}\right]}\right),
$$

and,

$$
\beta_{<H>}=\beta_{<R>}=\frac{1}{4} \cdot\left(1-\frac{1}{4 \cdot \ln \left[(4 K / 3)<V>^{1 / 3} / \tilde{h}_{0}\right]}\right) .
$$

By the above results,

$$
\lim _{\langle V>\rightarrow \infty} \beta_{H}=\lim _{<V>\rightarrow \infty} \beta_{R}=\frac{1}{4},
$$

wheareas

$$
\lim _{<V>\rightarrow \infty} \beta_{<V>}=\frac{3}{4},
$$

and

$$
\lim _{\langle V>\rightarrow \infty} \beta_{<P>}=-\frac{1}{4} .
$$

By the results in the Eqs. (51), (53) and (54), we see that the magnitudes of the effective $(<V>$-dependent) coarsening exponents are depressed below their asymptotic values, by a slowly changing logarithmic correction. The correction originates from the log in the denominator of the cluster dynamics equation (15), containing the "screening length" $\xi$. This depression of the exponents below their asymptotic values is the dominant correction to scaling at long times, i.e., in the large $\langle V\rangle$ limit. On the other 
hand, as we will reveal in the following section [Sec. 5.7], at early times, the dominant correction to scaling comes from the breakdown of the Eq. (44), i.e., of the classical Young-Laplace model. In this early time regime the Eq. (44) needs to replaced by the exact forms of $P_{\text {stat }}(V), H_{\text {stat }}(V)$, and $R_{\text {stat }}(V)$ obtained in Ch. 4 . The resulting coarsening exponents are discussed in the next section. Here we note that the breakdown of the Young-Laplace model at early times yields the coarsening exponents that are actually above their asymptotic values, in contrast to the effect of the above discussed logarithmic correction (due to the screening length $\xi$ ) which tends to depress the coarsening exponents below their asymptotic values.

\subsection{Effective exponents beyond the Young-Laplace model}

In this Section, we will use the results of the previous sections of this chapter to analytically explain the findings of our simulations in Chapter 3. Main theoretical results will be the effective cluster growth coarsening exponents calculated as functions of the cluster average volume $\langle V\rangle$ at time $t$. Furthermore, we will compare our analytic results for the cluster coarsening exponents with the corresponding results obtained from our numerical simulations of Chapter 3. The comparison will show a very good agreement between our analytic results and the corresponding results for the effective exponents

obtained from our simulations. This agreement constitutes the final proof of our assertion that the long range de-wetting interactions are responsible for the significant early time departures from the asymptotic predictions based on the classical YoungLaplace model.

Our analytic calculation of the effective coarsening exponents employs the iterative procedure introduced in Eq. (33), yielding the exponent $\beta_{V \max }$. The exponents $\beta<V>, \beta<P>$,

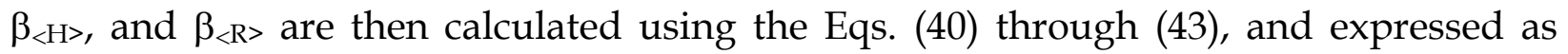


functions of the average cluster volume $\langle V\rangle$, as detailed in Sec. 5.5. In these analytic calculations, the central inputs are the form of quasi-static cluster pressure $P_{\text {stat }}(V)$, cluster height $H_{\text {stat }}(V)$, and cluster base radius $R_{\text {stat }}(V)$, all obtained in $C h .4$ for our rescaled model [Sec. 2.4], for the cases $\omega=1, \omega=2$, and $\omega=6$; see Figs. 2-4 of Ch. 4 and Appendix to Ch. 4 . In the figures that follow, we display our analytic results for $\beta<v>$, $\beta_{<P>}, \beta_{<H>}$, and $\beta_{<R>}$ as functions of $\langle V>$, for the cases $\omega=1, \omega=2$, and $\omega=6$; see Figs 2 through 5 . In the same set of figures, for comparison, we also display the corresponding results for $\beta_{<\mathrm{V}>}, \beta_{<\mathrm{P}>}, \beta_{<\mathrm{H}\rangle}$, and $\beta_{<\mathrm{R}>}$ as functions of $\langle V\rangle$, obtained from our simulations in Ch. 3. From the figures 2 through 5 we see that there are statistical fluctuations seen in the simulations results. Note however that these statistical fluctuations go around the corresponding analytic results for the exponents. By the figures 2 through 5, the agreement between the analytic and the simulations results for the exponents is very good, for all the cases $(\omega=1, \omega=2$, and $\omega=6)$ and for all the exponents $\beta<\mathrm{V}>, \beta<P>, \beta<H>$, and $\beta_{<R>\text {. }}$

We recall that our analytic theory contains a numerical proportionality constant $C$ [see Eq. (33), or Eq. (20) for the screening length]. This constant relates to the intercluster transport of material over nearly "dry" areas where the film thickness is very small. Thus, the $C$ should not depend much on the details of the de-wetting potential such as the value of $\omega$, which actually describes the asymptotic behavior of the potential for large film thicknesses occurring only in "wet" areas inside the clusters. We indeed find that simulations of the cases $\omega=1, \omega=2$, and $\omega=6$ can be all well fitted by our analytic theory using a single value of $C=1.60$ that has been employed in the figures of this chapter.

As evidenced by the figures 2-5, both the analytic theory and the simulations exhibit a significant effect of the actual value of the $\omega$ on the early time behavior of the effective exponents $\beta_{<\mathrm{V}>}, \beta_{<\mathrm{P}>}, \beta_{<H>}$, and $\beta_{<\mathrm{R}>}$ :Larger is the $\omega$, smaller is the difference between effective exponents and their asymptotic values in Eqs. (55-57). Indeed, for a large $\omega=6$ (essentially, a short range de-wetting potential), the early time range exponent 
$\beta_{<\mathrm{H}>}$ is about 0.28 , so it is close to the asymptotic $\beta_{<\mathrm{H}>}=0.25$. On the other hand, for long-range potentials with for $\omega=1$ and $\omega=2$, the early time range exponent $\beta_{<H}>$ is about 0.33 , i.e., it deviates significantly from the asymptotic $\beta_{<\mathrm{H}>}=0.25$. We recall that the cases $\omega=1$ and $\omega=2$ are the physically interesting cases, corresponding to van der Waals interaction $(\omega=2)$, and to fermionic Casimir interaction $(\omega=1)$; see Chapters 1 and 2 discussions. Experimentally, such an effective early-time exponent, measured to be $\beta_{H}^{e f f} \approx 0.33$, has been indeed reported for Sn clusters on silicon, see Ref. [13] of Ch. 1 . Our analytic theory, in combination with simulations, explains these experimental findings in terms of long range de-wetting potentials.

With increasing time, i.e., cluster average volume $\langle V\rangle$, the effective coarsening exponents do approach their asymptotic values Eqs. (55-57) predicted by YoungLaplace model. This approach is however non-monotonous for the exponents $\beta<v>$, $\beta_{<H>}$, and $\beta_{<R>}$, as can be seen in the figures 6 through 8 . In this figure, we display the analytically calculated effective exponents in a volume $\langle\mathrm{V}\rangle$ range that is much wider than in Figs. 2-5 so one can see better the approach to the asymptotic values of the exponents which are 0.75 for $\beta_{<\mathrm{V}>},-0.25$ for $\beta_{<\mathrm{P}>}, 0.25$ for $\beta_{<H>}$, and 0.25 for $\beta_{<\mathrm{R}>}$. By this figure, for example, the exponent $\beta_{<\mathrm{H}}$ is bigger than $1 / 4$ at early times, and it crosses $1 / 4$ at a finite time. After this, the $\beta_{<\mathrm{H}}>$ becomes smaller than $1 / 4$, and it goes through a minimum value $(<1 / 4)$ at a finite time. After this instant of time, the $\beta_{<\mathrm{H}>}$ increases to reach the asymptotic value of $1 / 4$ at infinite time. The early regime with $\beta_{<\mathrm{H}>}>1 / 4$ is dominated by the breakdown of the Young-Laplace model. On the other side, the late time regime with $\beta_{<\mathrm{H}}<1 / 4$ is dominated by the screening length effects discussed in Sec. 5.6. 

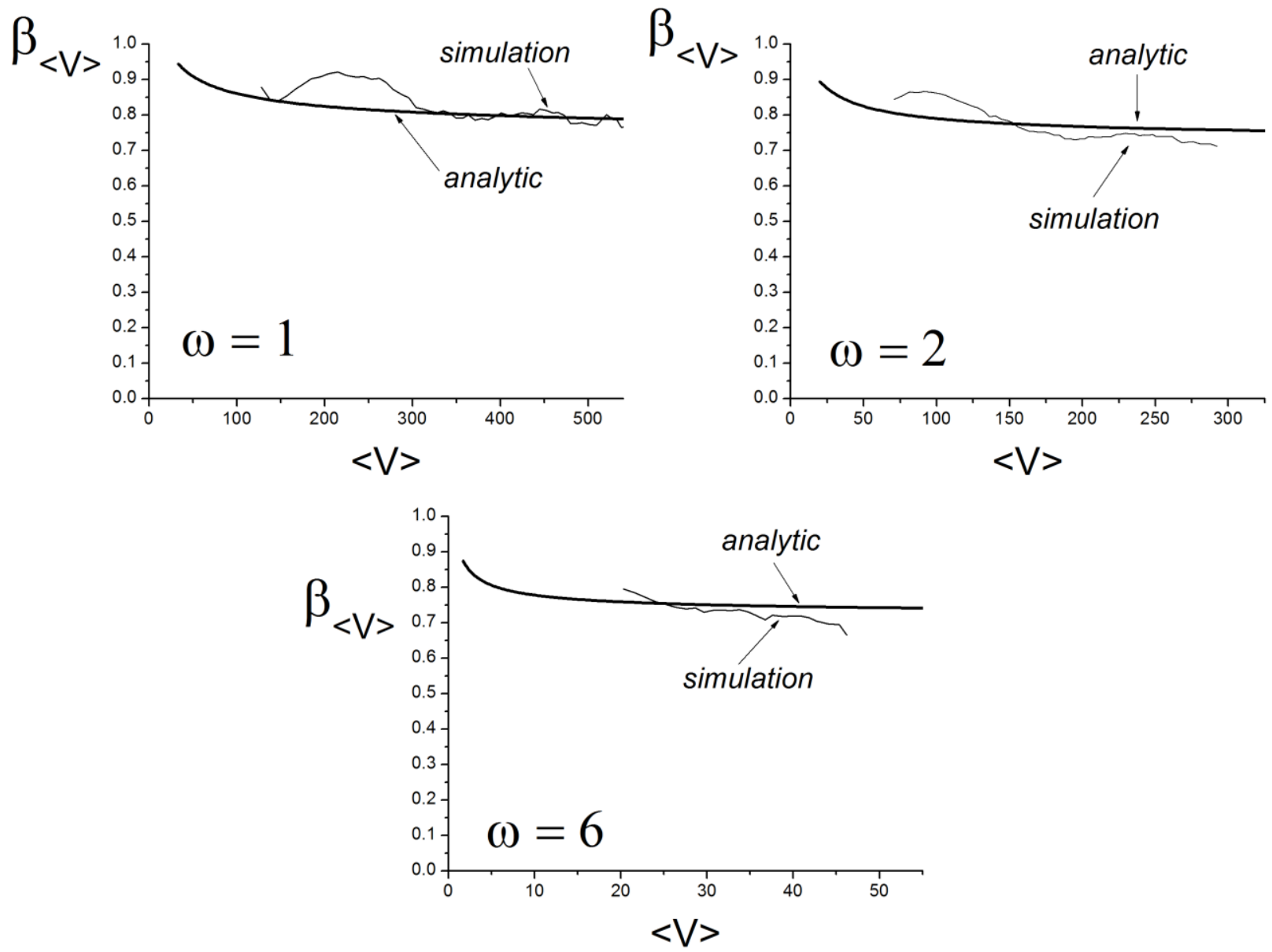

Fig. 2. Analytic results versus the corresponding simulation results for the effective coarsening exponent for the growth of cluster volume, $\beta<V\rangle$ as function of the average cluster volume $\langle V\rangle$. We recall that the asymptotic value of $\beta<\mathrm{V}>$ is 0.75 for infinite $<\mathrm{V}>$. 


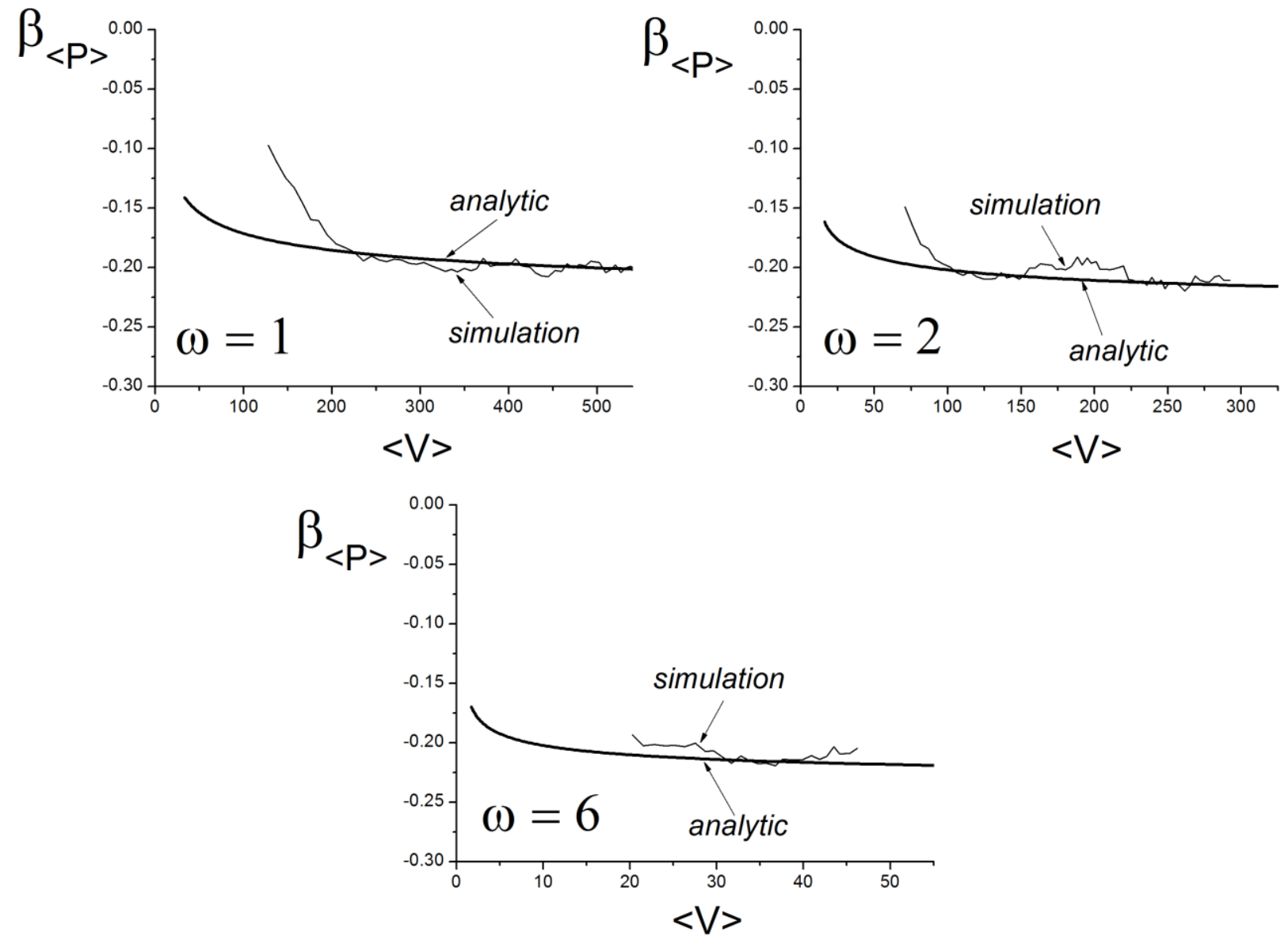

Fig. 3. Analytic results versus the corresponding simulation results for the effective coarsening exponent for the dynamics of cluster pressure, $\beta<P>$ as function of the average cluster volume $\langle V>$. We note that the asymptotic value of $\beta<\mathrm{P}>$ is -0.25 for infinite $<\mathrm{V}>$. 


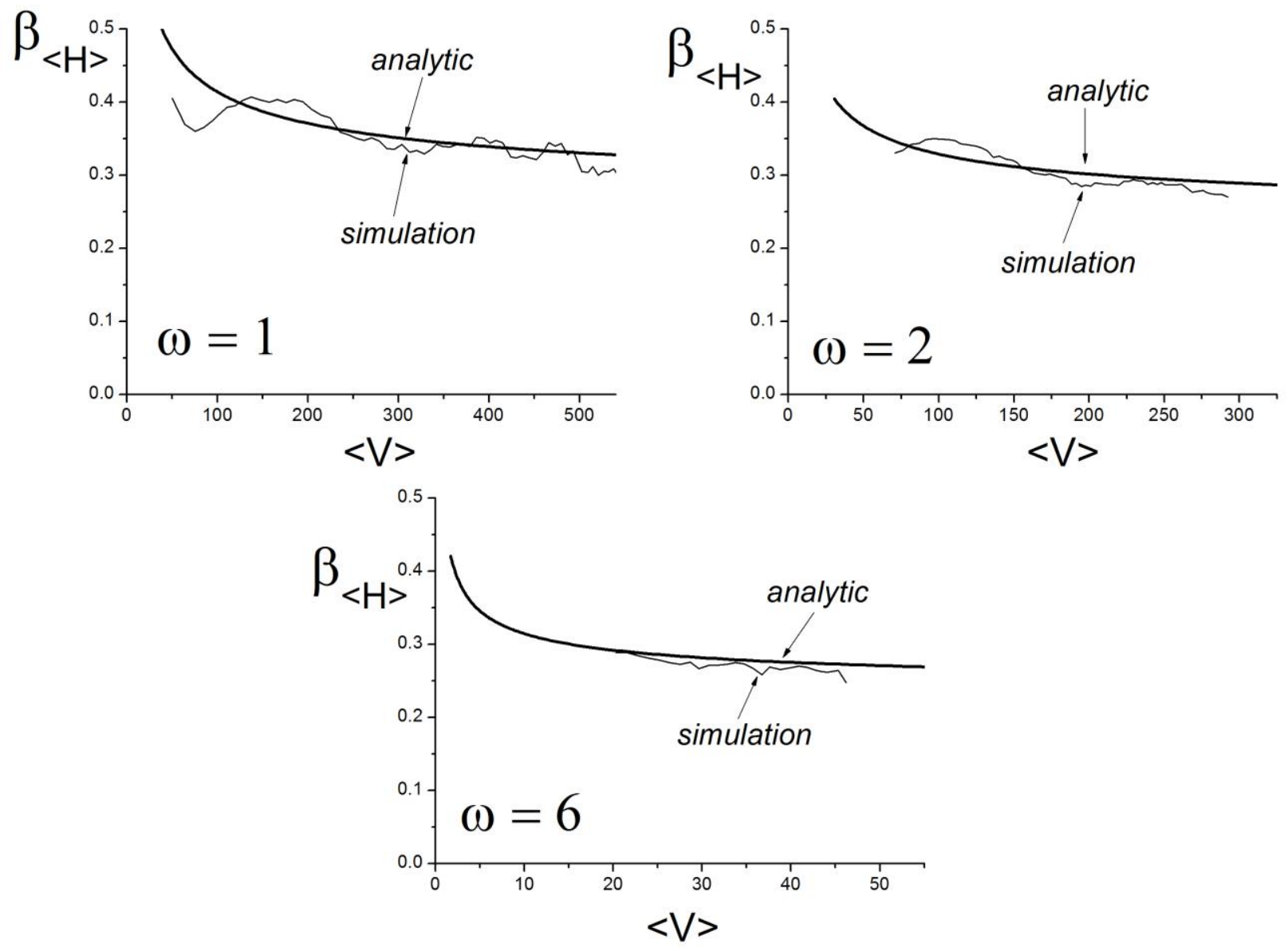

Fig. 4. Analytic results versus the corresponding simulation results for the effective coarsening exponent for the growth of cluster height, $\beta_{<\mathrm{H}\rangle}$ as function of the average cluster volume $\langle V\rangle$. We recall that the asymptotic value of $\beta_{<\mathrm{H}>}$ is 0.25 for infinite $<\mathrm{V}>$. 

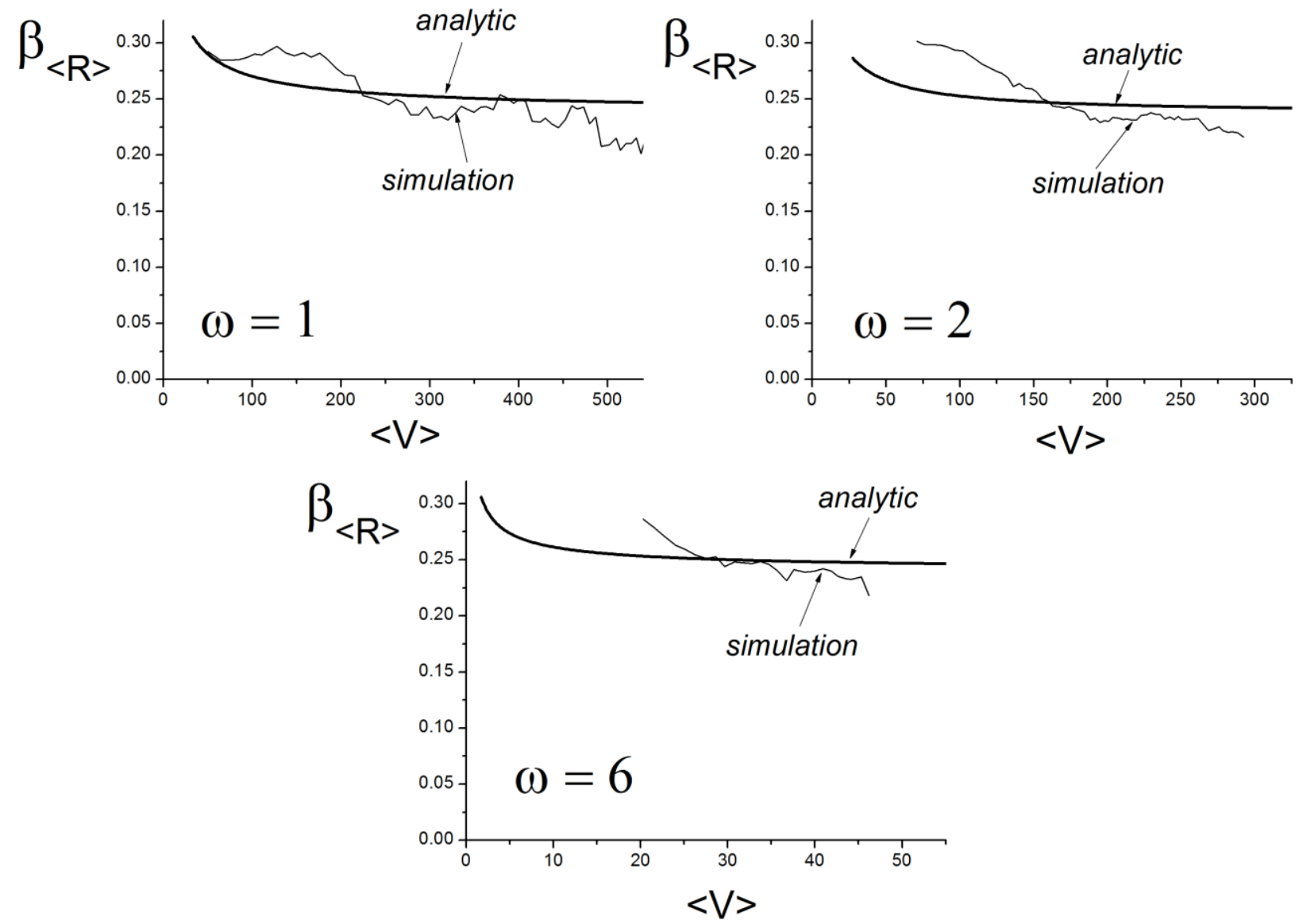

Fig. 5. Analytic results versus the corresponding simulation results for the effective coarsening exponent for the growth of cluster base radius, $\beta_{<R>}$ as function of the average cluster volume $\langle V\rangle$. We recall that the asymptotic value of $\beta_{<R}>$ is 0.25 for infinite $<V>$. 

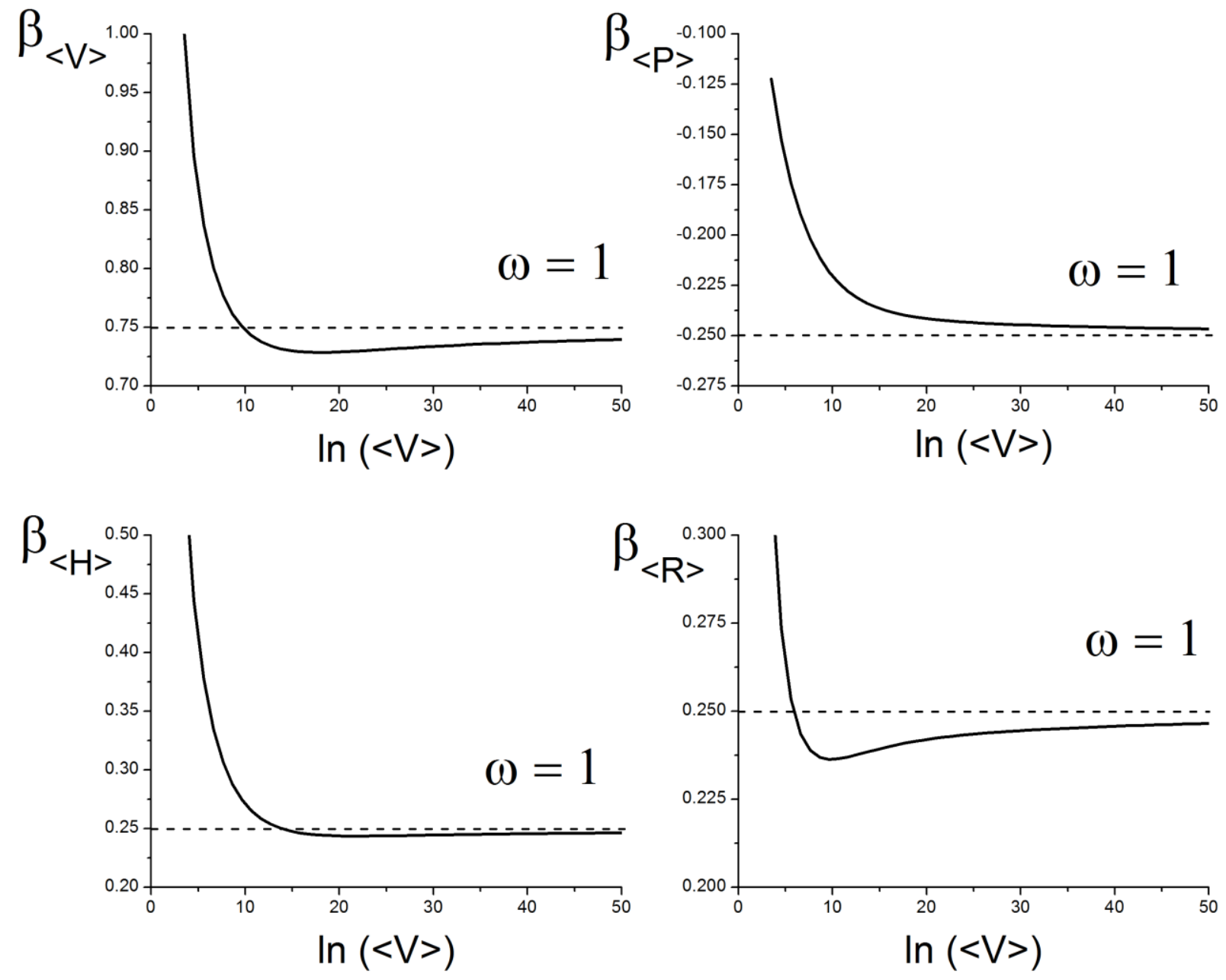

Fig. 6. Analytic results for the effective coarsening exponents $\beta<V>, \beta<P>, \beta_{<H>}$, and $\beta_{<R>}$ as functions of the average cluster volume $\langle V\rangle$, for the rescaled model with $\omega=1$. In this figure, we display the exponents in a volume $\langle\mathrm{V}\rangle$ range that is much wider than in figs. $2-5$ so one can see the better the approach to the asymptotic exponents values, which are 0.75 for $\beta<V>,-0.25$ for $\beta<P>, 0.25$ for $\beta<H>$, and 0.25 for $\beta_{<R>\text {. }}$ 

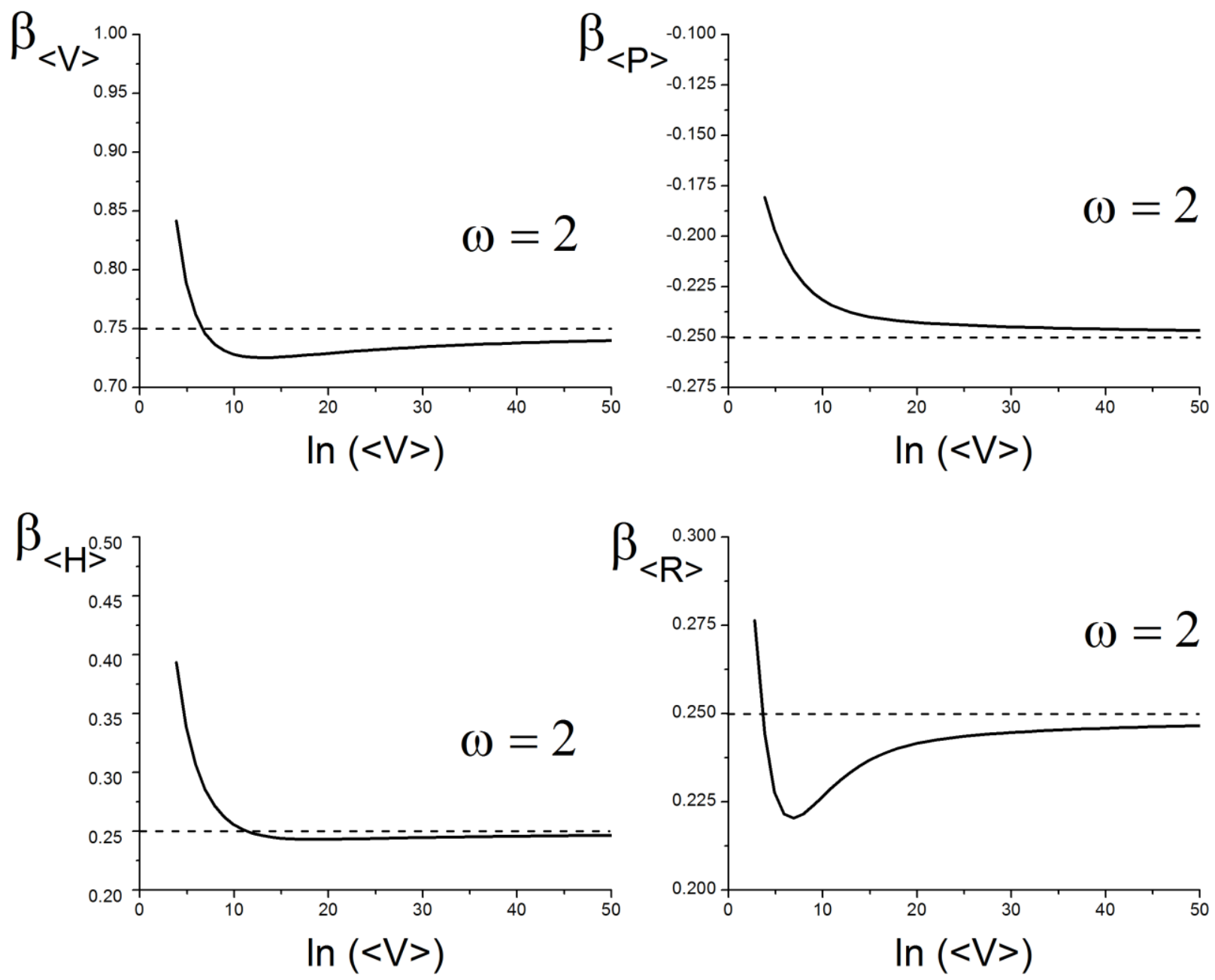

Fig. 7. Analytic results for the effective coarsening exponents $\beta_{<V>}, \beta<P>, \beta_{<H>}$, and $\beta_{<R>}$ as functions of the average cluster volume $\langle V\rangle$, for the rescaled model with $\omega=2$. In this figure, we display the exponents in a volume $\langle\mathrm{V}\rangle$ range that is much wider than in figs. 2-5 so one can see the better the approach to the asymptotic exponents values, which are 0.75 for $\beta<V>,-0.25$ for $\beta<P>, 0.25$ for $\beta<H>$, and 0.25 for $\beta_{<R>\text {. }}$ 

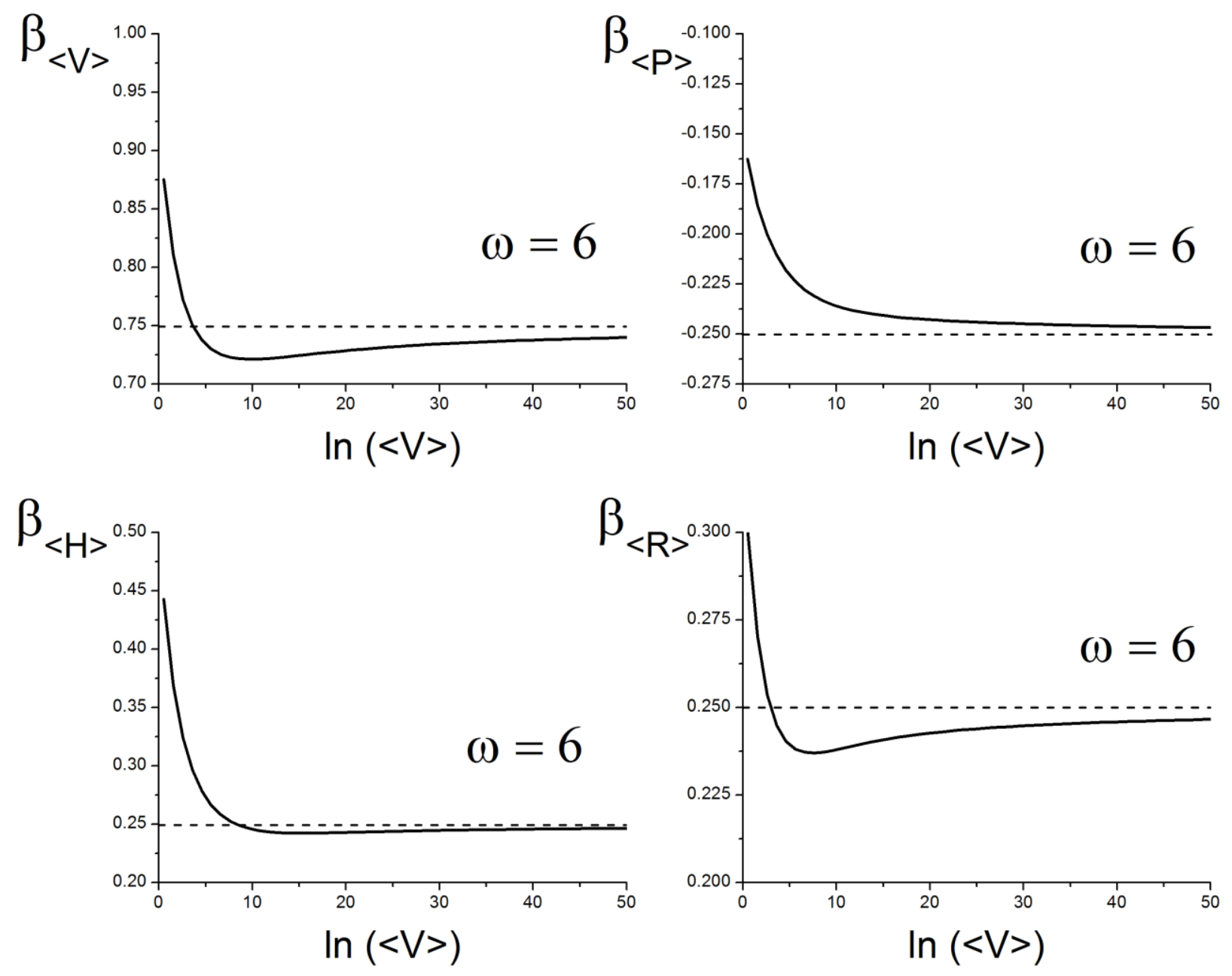

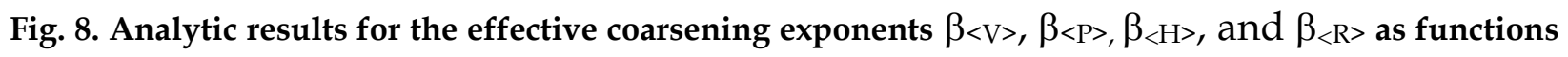
of the average cluster volume $\langle V\rangle$, for the rescaled model with $\omega=6$. In this figure, we display the exponents in a volume $\langle V\rangle$ range that is much wider than in figs. 2-5 so one can see the better the approach to the asymptotic exponents values, which are 0.75 for $\beta<V>,-0.25$ for $\beta<P>, 0.25$ for $\beta<H>$, and 0.25 for $\beta_{<R>\text {. }}$ 


\subsection{References}

[1] I.M. Lifshits and V.V. Slyozov, Sov. Phys. JETP 35 (1959) 331.

[2] K. Shorlin, S. Krylov, and M. Zinke-Allmang, Physica A 261 (1998) 248.

[3] B.K. Chakraverty, J. Phys. Chem. Solids 28 (1967) 2401. 


\section{Thesis Summary}

- We used numerical simulations of our interface dynamics model to explore the role played by the long-range de-wetting interactions on the character of the cluster coarsening dynamics.

- We find that these interactions produce significant early time departures from the asymptotic predictions based on the classical Young-Laplace model.

- To address this phenomenon, we first provided a theoretical description of quasi-static (slowly evolving) clusters that goes beyond the classical Young-Laplace model.

- Next, we used this description to develop a novel and powerful cluster dynamics theory capable to calculate effective coarsening exponents and thus analytically explain the findings of our simulations.

We have theoretically proved, by numerical simulations and analytic methods, that the long range nature of de-wetting forces produces long lasting early-time departures from the asymptotic (Young-Laplace) power laws, with the effective exponent $\beta_{<H>}>0.25$ for the growth of cluster height. Experimentally, such an effective early-time exponent, measured to be $\beta_{<H>} \approx 0.33$, has been indeed reported for Sn clusters on silicon. Our analytic theory, in combination with simulations, explains these experimental findings in terms of long range de-wetting potentials. 